\title{
Foraminifera of the North Pacific Ocean
}

GEOLOGICAL SURVEY PROFESSIONAL PAPER 766

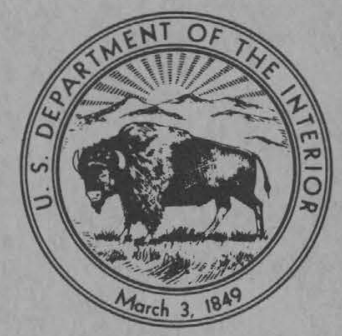





\section{Foraminifera of the North Pacific Ocean}

By PATSY B. SMITH

GE OLGICAL S URVEY PROFESSIONAL PAPER 766

A systematic study of Foraminifera from lat $25^{\circ}$ to $55^{\circ} \mathrm{N}$.

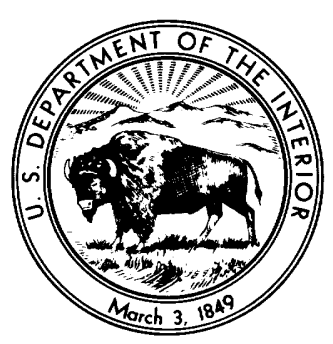




\title{
UNITED STATES DEPARTMENT OF THE INTERIOR \\ ROGERS C. B. MORTON, Secretary
}

\section{GEOLOGICAL SURVEY}

\author{
V. E. McKelvey, Director
}

Library of Congress catalog-card No. 72-600385

For sale by the Superintendent of Documents, U.S. Government Printing Office, Washington, D.C. 20402

Price: Paper cover - 85 cents, domestic postpaid; 60 cents, GPO Bookstore. $\quad$ Stock No. 2401-00295 


\section{CONTENTS}

Abstract

Introduction ----Acknowledgments

Previous work

Procedure

Environment of the North Pacific

Foraminifera in grab samples and in top 2 centimeters of cores

Foraminifera below top 2 centimeters of cores -

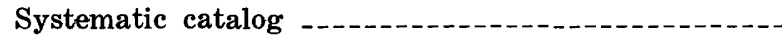

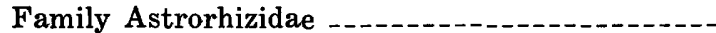
Genus Rhabdammina Genus Rhizammina

Genus Marsipella

Genus Bathysiphon --.--

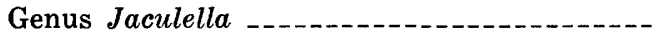

Genus Hyperammina -.........

Family Saccamminidae _................... Genus Psammosphaera -...-...Genus Saccammina -

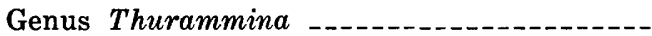

Family Ammodiscidae -......Genus Glomospira

Family Hormosinidae .

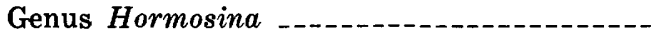
Genus Reophax

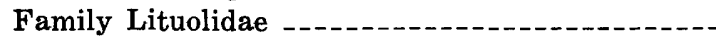
Genus Adercotryma ........................ Genus Cyclammina Genus Alveolophragmium -.......-..-Genus Ammobaculites -........-...-. Genus Ammomarginulina _.............. Genus Placopsilina

Family Textulariidae Genus Spiroplectammina Genus Bigenerina

Family Trochamminidae -..-Genus Trochammina -..-..-..-. Genus Cystammina -..-_-_._Genus Tritaxis _................

Family Ataxophragmiidae Genus Globotextularia Genus Dorothia Genus Eggerella

Family Nubeculariidae -...-. Genus Ophthalmidium

\begin{tabular}{|c|c|c|}
\hline age & & Page \\
\hline 1 & Systematic catalog-Continued & \\
\hline 1 & Family Miliolidae & \\
\hline 4 & Genus Quinqueloculina & 19 \\
\hline 4 & Genus Pyrgo - & 19 \\
\hline 6 & Genus Miliolinella & 19 \\
\hline 6 & Genus Ammomassilina & \\
\hline & Family Turrilinidae & \\
\hline 7 & Genus Buliminella & \\
\hline 11 & Family Bolivinitidae & \\
\hline 12 & Genus Bolivina & \\
\hline 12 & Genus Bulimina & \\
\hline 12 & Genus Globobulimina & \\
\hline 12 & Family Uvigerinidae & \\
\hline 12 & Genus Uvigerina & \\
\hline 12 & Genus Angulogerina & \\
\hline 12 & Family Discorbidae & \\
\hline 12 & Genus Epistominella & \\
\hline 13 & Family Elphidiidae & \\
\hline 13 & Genus Elphidium & \\
\hline 13 & Genus Elphidiella & \\
\hline 13 & Family Globorotaliidae & \\
\hline 13 & Genus Globorotalia & \\
\hline 13 & Family Globigerinidae & \\
\hline 13 & Genus Globigerina & \\
\hline 13 & Genus Candeina & \\
\hline 13 & - & \\
\hline 14 & s - - & \\
\hline 14 & dae - & \\
\hline 14 & Genus Cassidulina & \\
\hline 15 & Genus Ehrenbergina & \\
\hline 16 & Family Involutinidae & \\
\hline 16 & Genus Involutina & \\
\hline 16 & Family Nonionidae & \\
\hline 16 & Genus Nonion & \\
\hline 16 & s Nonionella & \\
\hline 16 & Genus Pullenia & \\
\hline 17 & Family Alabaminidae & \\
\hline 17 & Genus Gyroidina & \\
\hline 18 & Family Anomalinidae & \\
\hline 18 & Genus Anomalina & \\
\hline 18 & Genus Cibicidoides & \\
\hline 18 & Genus Melonis & \\
\hline 18 & Family Ceratobuliminidae & \\
\hline 18 & $\operatorname{lin} \boldsymbol{a}_{-1}$ & \\
\hline 18 & - & \\
\hline 18 & & \\
\hline
\end{tabular}




\section{ILLUSTRATIONS}

[Plates 1-4 follow index]

Plates 1-4. Illustrations of Foraminifera.

Figure 1. Location of 1961 Pioneer stations -

2. Distribution of marine deposits in the North Pacific Ocean .....-

3. Distribution of calcium carbonate in the ocean deposits -

4. Diagram showing depth of water for stations along long. $160^{\circ} \mathrm{W}$

5. Diagram showing depth of water for stations along long. $180^{\circ} \mathrm{W}$ -

TABLES 


\title{
FORAMINIFERA OF THE NORTH PACIFIC OCEAN
}

\author{
By Patsy B. Smith
}

\section{ABSTRACT}

In 1961,33 cores were collected by the U.S. Coast and Geodetic Survey ship Pioneer. The Foraminifera of these cores, Holocene and older, indicate a wide faunal diversity. Most are arenaceous. In the deep parts of the central North Pacific, faunas are poor, both in number of specimens and species, but in the Aleutian Trench and on the adjacent island shelf, they are rich. A systematic catalog of the 85 species found accompanies the descriptions and information on distribution.

\section{INTRODUCTION}

During 1961, core samples were obtained by the U.S. Coast and Geodetic Survey ship Pioneer. Samples were taken between lat $25^{\circ}$ and $55^{\circ} \mathrm{N}$. and long $160^{\circ}$ to $180^{\circ} \mathrm{W}$. Water depth ranges from 76 to 7,230 meters (table 1). Samples were taken primarily along two north-south traverses through the midPacific that cross the Aleutian Trench at the north. Distribution of samples is shown in figure 1.

TABLE 1.-Position, depth, and content of core samples and the abundance of constituents, estimated in percent [C, core-catcher sample; other coarse organic fragments, estimated abundance in percent, given in parentheses]

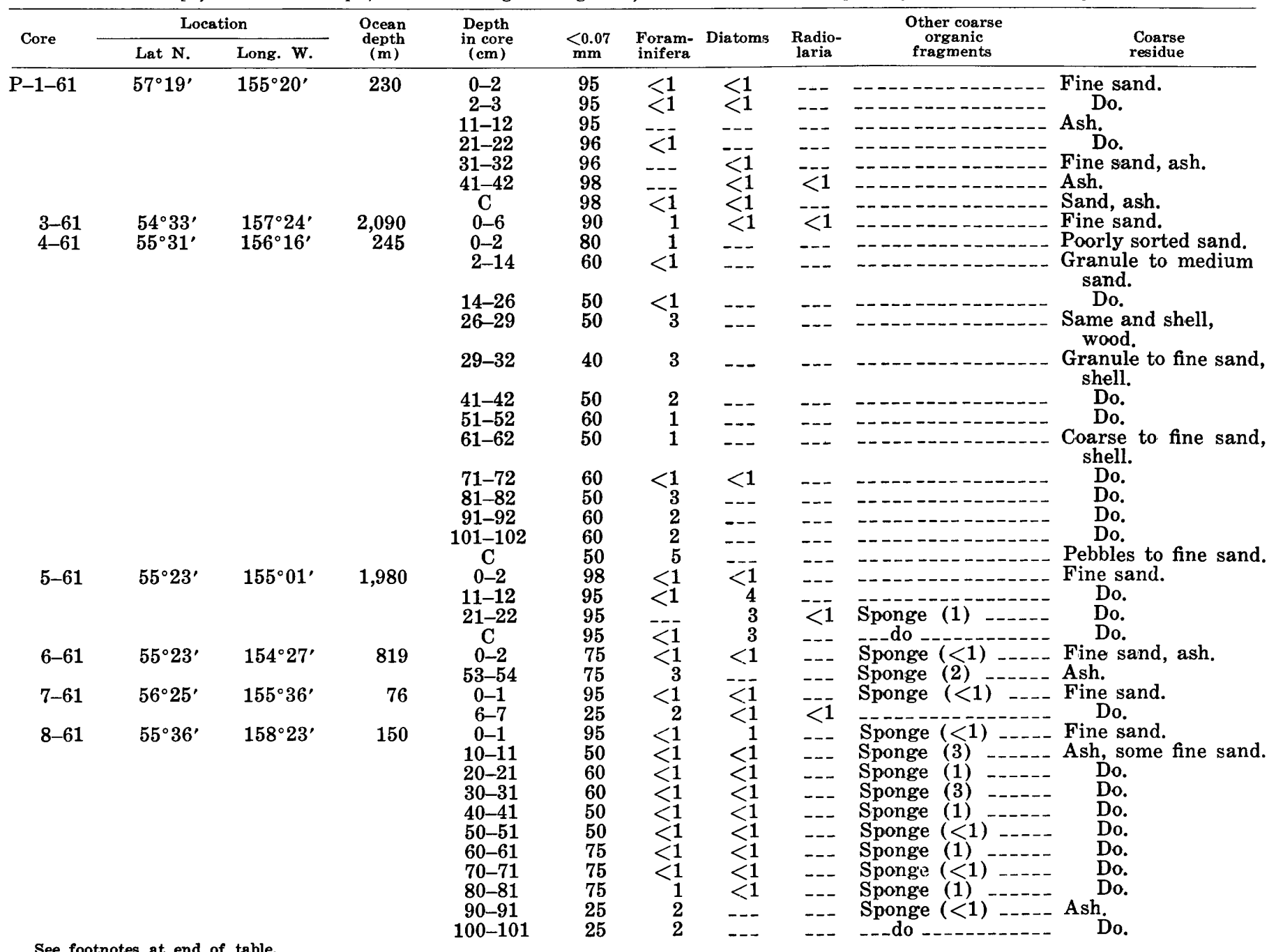


TABLE 1.-Position, depth, and content of core samples and the abundance of constituents, estimated in percent-Continued

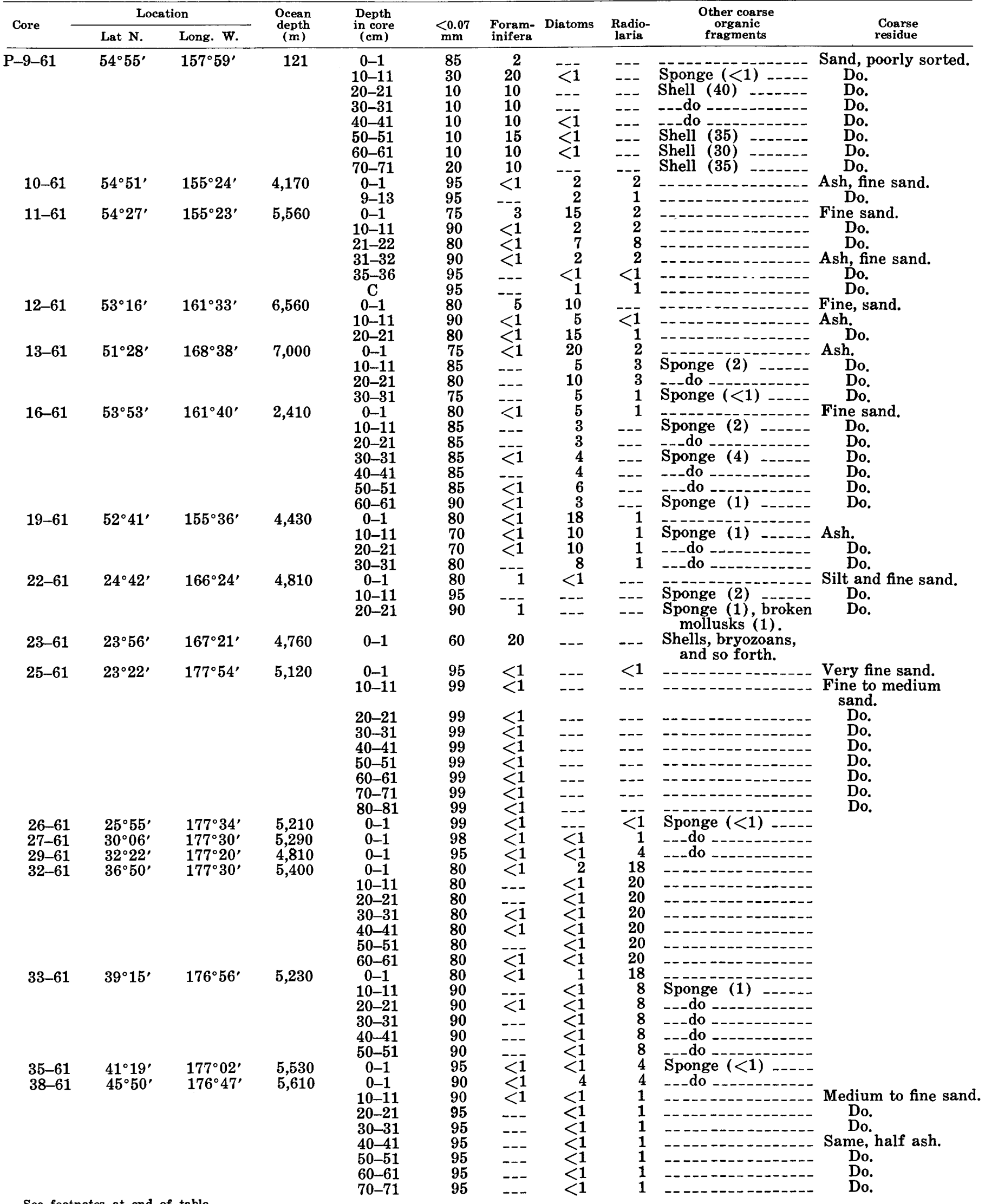


INTRODUCTION

TABLE 1.-Position, depth, and content of core samples and the abundance of constituents, estimated in percent-Continued

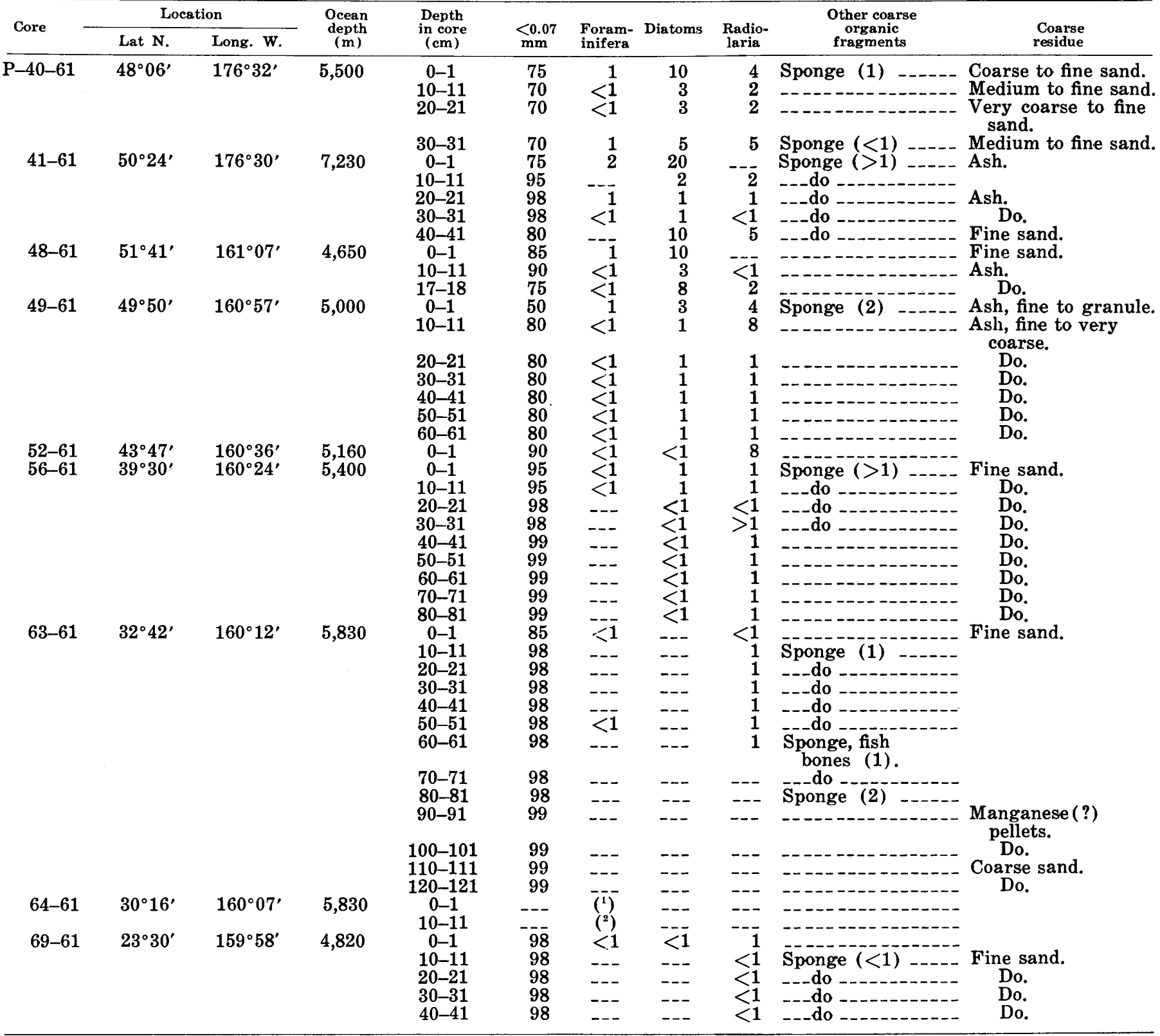

${ }^{1}$ Incompletely washed, few Foraminifera.

2 Incompletely washed, no Foraminifera.

Examination of faunas from the cores showed that, in general, species are sparsely represented but are diverse and mostly arenaceous. No new species are described. Sixty of the 85 species found were illustrated in the Challenger reports (Brady, 1884; Barker, 1960). The few calcareous specimens present in the deep cores were probably alive at the time of collection, even though not all were stained by rose bengal. The likelihood is not great that such specimens could survive for long after death in their calcite-undersaturated environment.
Table 1 lists the position, depth of water, and percentage estimates of the constituents of the cores. Most of the material was finer than 0.07 millimeter and contained small percentages of Foraminifera, diatoms, Radiolaria, other organic fragments, and some inorganic residue.

This paper is mainly a catalog of species present and their relative abundance. The systematic cata$\log$ includes as complete synonymies as possible, for many of the species have been described from rare occurrences and their systematic descriptions had to 


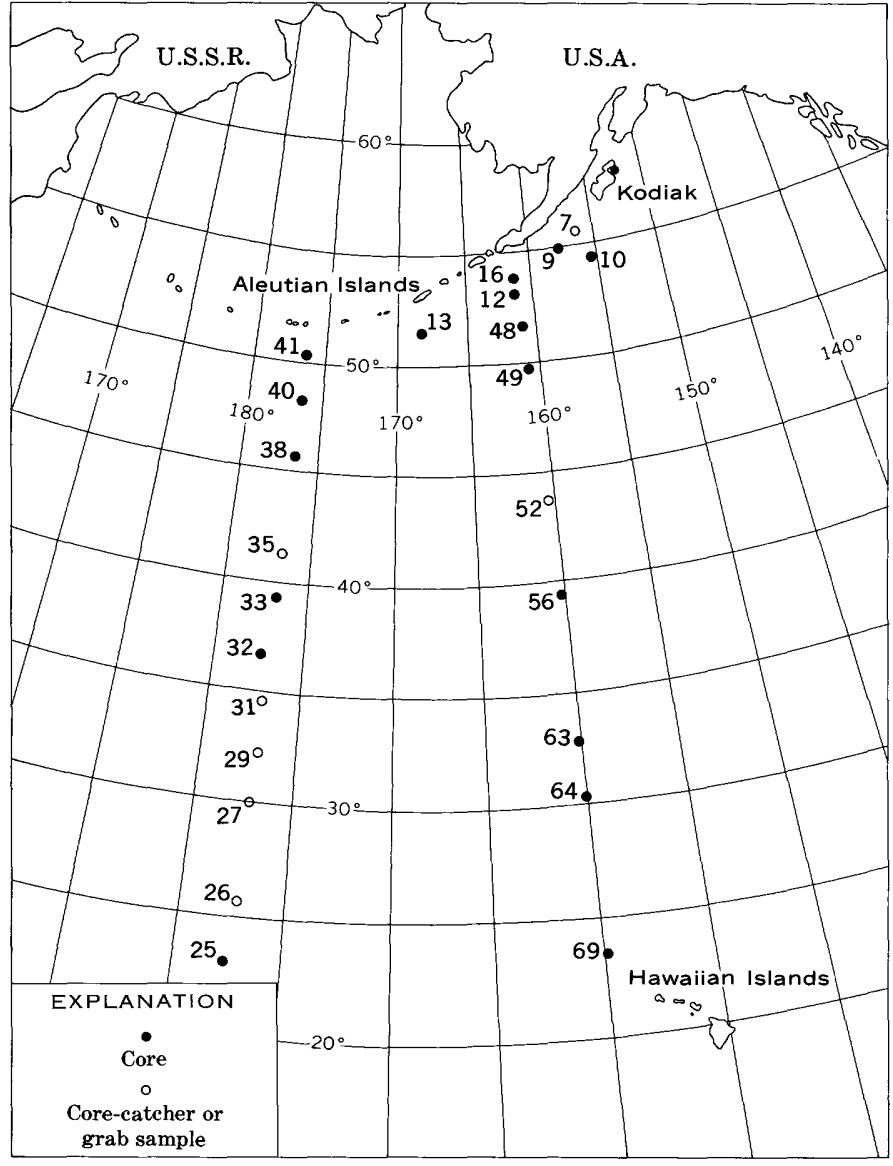

Figure 1.-Location of 1961 Pioneer stations. be assembled. Discussion of each species, its morphology, and distribution is included. The species are illustrated on plates 1-4.

\section{ACKNOWLEDGMENTS}

Credit is here given to Capts. William Dean, Horace Conerly, and Harley Nygren of the National Ocean Survey (formerly Coast and Geodetic Survey) for supervising the coring. George W. Moore of the U.S. Geological Survey logged and sampled the cores and collected the grab samples.

\section{PREVIOUS WORK}

Published records of bottom sediments and Foraminifera from the Pacific Ocean north of lat $20^{\circ} \mathrm{N}$. are sparse. The Challenger reports include several samples from this area. The report of the seventh cruise of the Carnegie (Revelle, 1944) includes many more samples and also a comprehensive study of sediment type, the distribution of which is shown in figure 2. Figure 3, showing distribution of $\mathrm{CaCO}_{3}$, is taken from Lisitzen (1971).

Riedel and Funnell (1965) described several Tertiary cores from the area, and several reports of Tertiary faunas from the North Pacific (Bukry and others, 1971; Krashininnikov, 1971; Olson and Goll, 1970) have been published as results of the deep-sea drilling project of the Glomar Challenger.

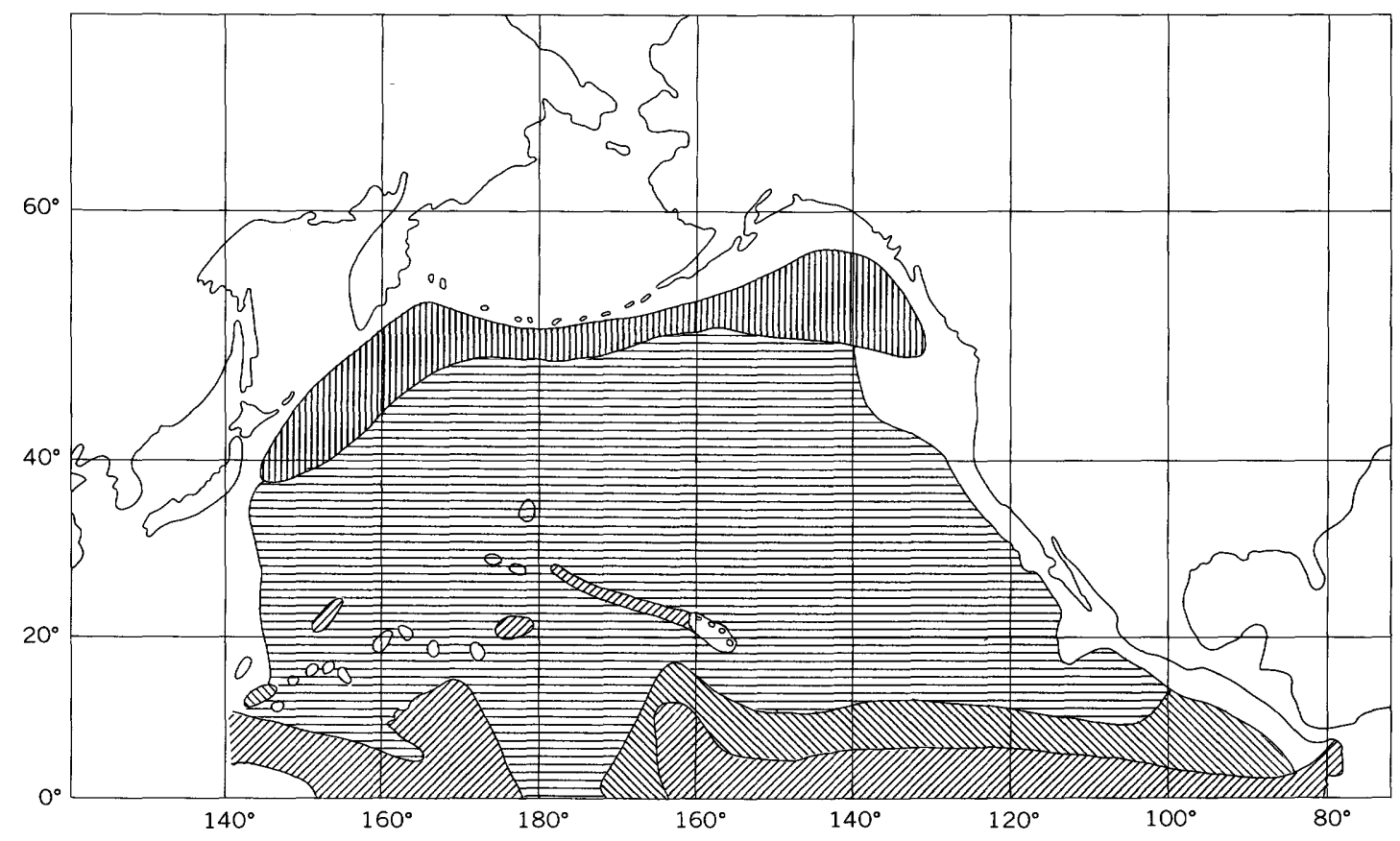

EXPLANATION
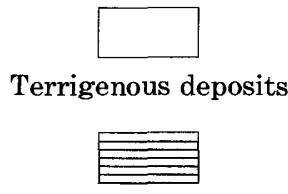

Red clay

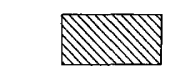

Radiolarian ooze

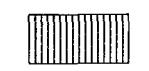

Diatom ooze

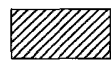

Globigerina ooze

Figure 2.-Distribution of marine deposits in the North Pacific Ocean (from Revelle, 1944). 


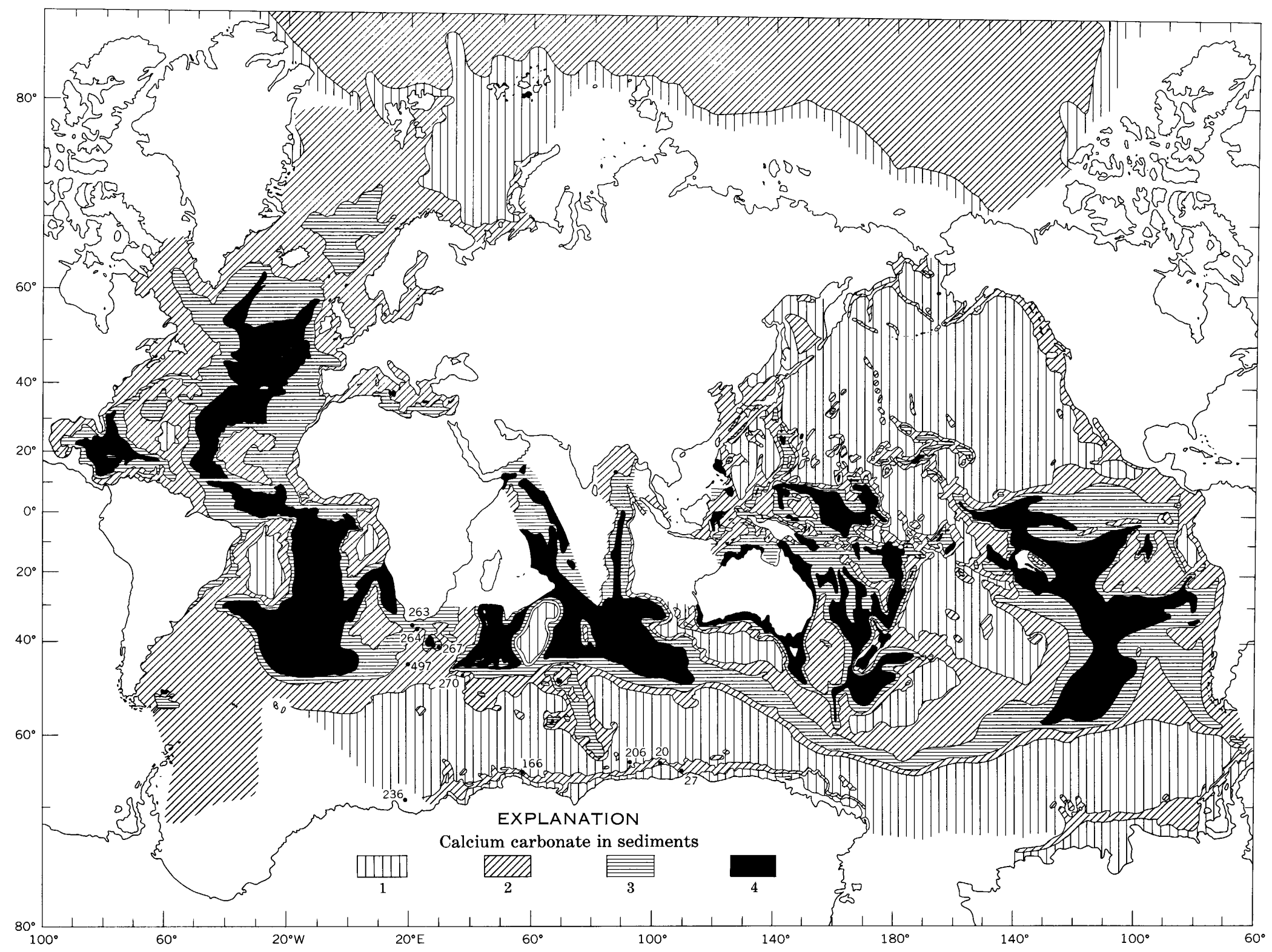

FIGURE 3-Distribution of calcium carbonate in the ocean deposits (from Lisitzen, 1971). 1, <1 percent; 2, 1-30 percent; 3, 30-70 percent; $4,>70$ percent. 


\section{PROCEDURE}

The top 2 centimeters of each core was preserved in alcohol and stained with rose bengal; the remainder of each core was examined at $10-\mathrm{cm}$ intervals.

Samples were washed on a 200 -mesh screen, and all Foraminifera were picked. The number of specimens indicated on pages 7-12 represents the actual number of specimens counted.

\section{ENVIRONMENT OF THE NORTH PACIFIC}

Bottom-water temperature in most parts of the area covered by this report ranges from $1^{\circ}$ to $2^{\circ} \mathrm{C}$. The sediment types are shown in figure 2 , taken from Revelle (1944). Samples directly south of the Aleutian Islands are composed of terrigenous material; those farther south, to latitude $50^{\circ} \mathrm{N}$., are markedly rich in diatoms (see table 1); and those still farther south are characterized by red clay. None of the samples are far enough south to reach areas high in $\mathrm{CaCO}_{3}$. (See fig. 3.)

Samples taken in fairly shallow water, north of the Aleutian Trench, have a high percentage of calcareous forms. Table 2 is a compilation of species

TABLE 2.-Distribution of Foraminifera in the North Pacific

\begin{tabular}{|c|c|c|c|}
\hline & $\begin{array}{l}\text { Aleutian } \\
\text { Terrace }\end{array}$ & $\begin{array}{c}\text { Aleutian } \\
\text { Trench }\end{array}$ & $\begin{array}{c}\text { Deep-sea } \\
\text { plain }\end{array}$ \\
\hline $\begin{array}{l}\text { Bolivina pseudoplicata } \\
\text { Buliminella basicostata } \\
\text { Elphidium magellanicum } \\
\text { Epistominella exigua } \\
\text { Fissurina sp }\end{array}$ & $\begin{array}{l}x \\
x \\
x \\
x \\
x\end{array}$ & $\begin{array}{l}------- \\
-------- \\
-------- \\
-------- \\
--------\end{array}$ & 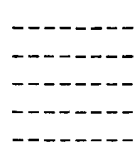 \\
\hline $\begin{array}{l}\text { Nonionella auricula } \\
\quad \text { bradyi } \\
\text { Rosalina sp } \\
\text { Virgulina cf. V. complanata } \\
\text { Globigerina bulloides }\end{array}$ & $\begin{array}{l}x \\
x \\
x \\
x \\
x\end{array}$ & $\begin{array}{l}-------- \\
-------- \\
-------- \\
-------- \\
--------\end{array}$ & 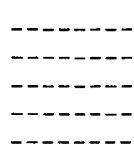 \\
\hline $\begin{array}{l}\text { Pseudogaudryina atlantica } \\
\text { Angulogerina angulosa } \\
\text { Bolivina decussata } \\
\text { Cassidulina crassa } \\
\quad \text { teretis } \\
\quad \text { tortuosa }\end{array}$ & $\begin{array}{l}x \\
x \\
x \\
x \\
x \\
x\end{array}$ & 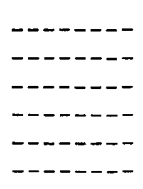 & 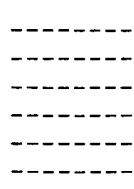 \\
\hline $\begin{array}{l}\text { Cibicides lobatulus } \\
\quad \text { refulgens } \\
\text { Nonion scaphum } \\
\text { Uvigerina cushmani } \\
\text { Tritaxis conica }\end{array}$ & $\begin{array}{l}x \\
x \\
x \\
x\end{array}$ & $\begin{array}{l}-------- \\
-\cdots------ \\
-------- \\
-------- \\
--------\end{array}$ & 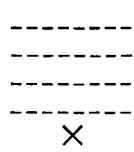 \\
\hline $\begin{array}{l}\text { Globotextularia anceps } \\
\text { Dorothia exilis } \\
\text { Eggerella bradyi } \\
\text { scabra } \\
\text { Ophthalmidium acutimargo } \\
\quad \text { pusillum }\end{array}$ & 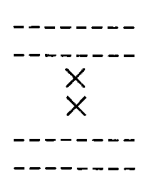 & $\begin{array}{c}x^{x} \\
x \\
-\end{array}$ & $\begin{array}{l}x \\
x \\
x \\
x \\
x \\
x\end{array}$ \\
\hline $\begin{array}{l}\text { Quinqueloculina sp } \\
\text { Pyrgo sp } \\
\text { Miliolinella subrotunda } \\
\text { Ammomassilina } \\
\quad \text { alveolinaformis } \\
\text { Bolivina robusta }\end{array}$ & $\begin{array}{c}----- \\
------ \\
-----\end{array}$ & $x^{------}$ & $\begin{array}{c}x \\
-------\end{array}$ \\
\hline
\end{tabular}

TABLE 2.-Distribution of Foraminifera in the North Pacific-Continued

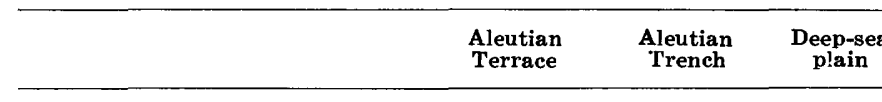

Bulimina aculeata

Globobulimina auriculata pacifica Uvigerina peregrina Epistominella exigua umbonifera

Elphidium incertum Elphidiella groenlandica Globorotalia inflata Globigerina bulloides -...-Candeina nitida

Cibicides bradyi Cassidulina subglobosa crassa

Ehrenbergina hystrix Involutina tenuis _........

Nonion labradoricum

Nonionella turgida Pullenia subcarinata ...-. Gyroidina lamarckiana -.. Anomalina globulosa

Cibicidoides cf. C. mundulus Melonis affine -.........pompilioides Hoeglundina elegans -..--. Rhabdamina abyssorum -..-

Rhizammina? sp

Marsipella cylindrica Bathysiphon discreta -..-Jaculella acuta Hyperammina spp

Psammosphaera rustica

Saccammina sphaerica

Thurammina papillata

Glomospira gordialis -..--

Hormosina globulifera

Reophax dentalinaformis -. diffugiformis _......... distans nodulosus ........... pilulifer scorpiurus _.......-. scotti excentricus ........ Adercotryma giomerata

Cyclammina cancellata -.trullissata

Alveolophragmium nitidum cf. $A$. nitidum --.ringens -....... scitulum -..-subglobosum _......weisneri _......-...... Alveolophragmium? sp -..-

Ammobaculites agglutinans agglutinans filaformis (smooth)

agglutinans filaformis (rough)

americanus -..-.

Ammomarginulina foliacea Placopsilina confusa

Spiroplectammina biformis_ Bigenerina minutissima

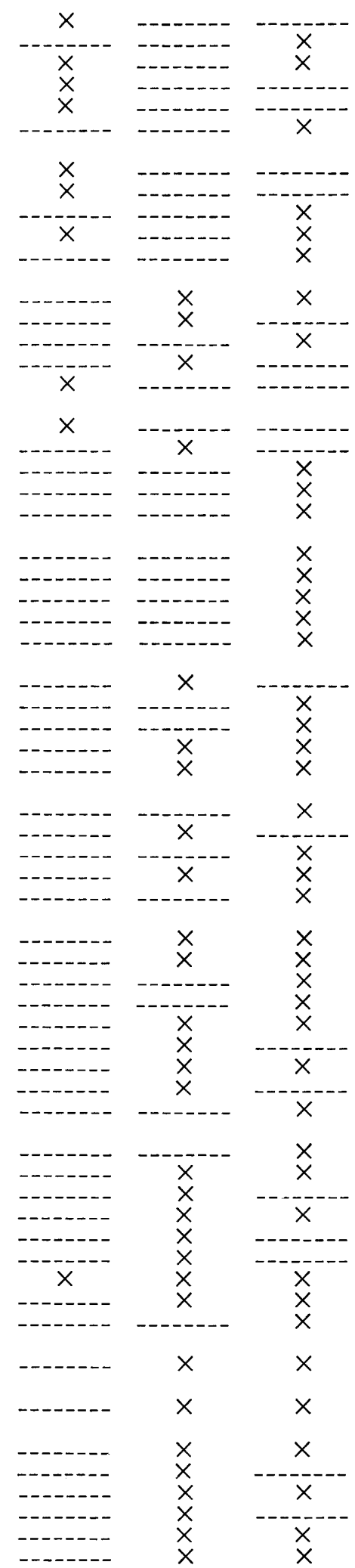


TABLE 2.-Distribution of Foraminifera in the North Pacific-Continued

\begin{tabular}{|c|c|c|c|}
\hline & $\begin{array}{c}\text { Aleutian } \\
\text { Terrace }\end{array}$ & $\begin{array}{c}\text { Aleutian } \\
\text { Trench }\end{array}$ & $\begin{array}{c}\text { Deep-sea } \\
\text { plain }\end{array}$ \\
\hline $\begin{array}{l}\text { Trochammina grisea } \\
\text { inflata }\end{array}$ & $\times$ & 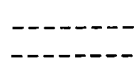 & $\stackrel{x}{x}$ \\
\hline kellettae & 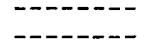 & $x$ & $x$ \\
\hline malovensis - & $---1-1$ & $x$ & --- \\
\hline cf. T. malovensis & ----- & $x$ & \\
\hline $\begin{array}{l}\text { nana } \\
\text { sp (chitinous) }\end{array}$ & --- & - & $x$ \\
\hline $\begin{array}{l}\text { sp. } \\
\text { nitida }\end{array}$ & $-\cdots---$ & $x$ & \\
\hline globigeriniformis & $x$ & $x$ & \\
\hline Cystammina galeata & ------- & $x$ & $x$ \\
\hline $\begin{array}{l}\text { Nodellum membranaceum -- } \\
\text { Haplophragmoides }\end{array}$ & - & & \\
\hline can & - & $x$ & $x$ \\
\hline & -..--- & $\dot{x}$ & -- \\
\hline elosi & ------ & & -- \\
\hline Psammosiph & - & & \\
\hline
\end{tabular}

that are present in three areas: the Aleutian Terrace, the Aleutian Trench, and the deep-sea plain. No indication of relative abundance is given. Samples from the trench itself have surprisingly large numbers of species and specimens, almost entirely arenaceous. One interesting aspect of the trench faunas is that the cement of several species is all or in part composed of pseudochitin, as though there simply was not enough calcitic cementing material available.

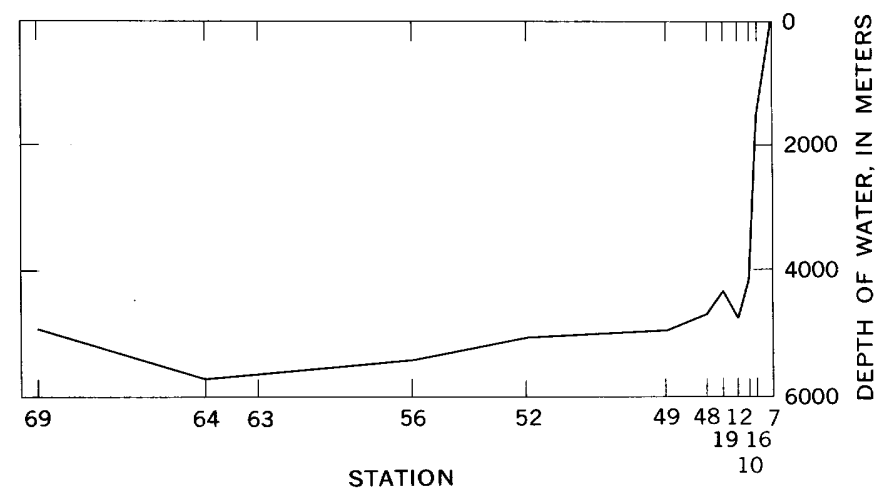

FIGURE 4.-Depth of water for stations along long. $160^{\circ} \mathrm{W}$.

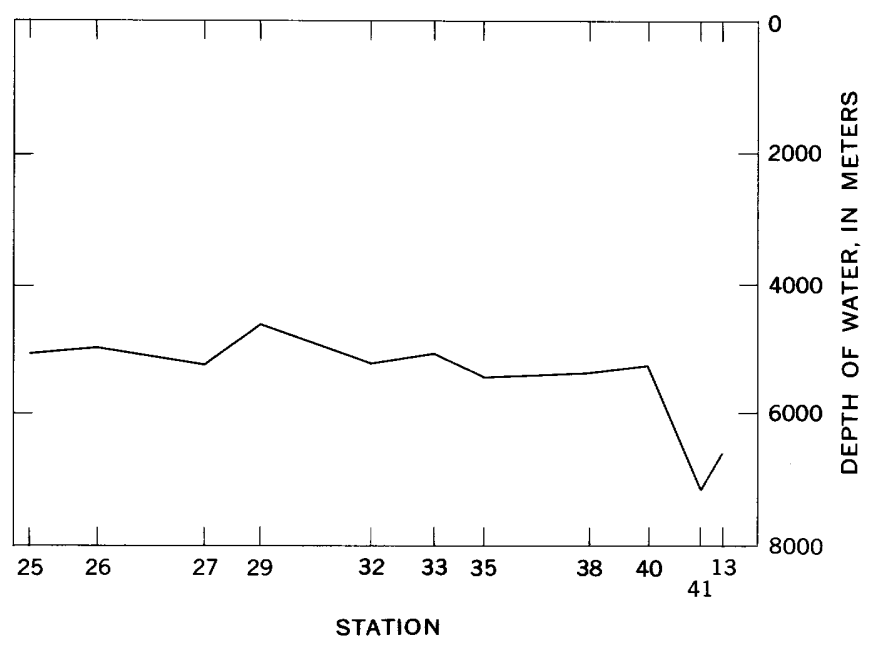

Figure 5.-Depth of water for stations along long. $180^{\circ} \mathrm{W}$.
Depths of the samples are plotted in figures 4 and 5 and show graphically the great depth from which most of these samples came. It is unusual to find Foraminifera at such great depths because of the low $\mathrm{CaCO}_{3}$ concentration.

Faunal lists of the top $2 \mathrm{~cm}$ of the cures are arranged from north to south. A separate list of faunas found below the surface are arranged serially by sample number. These fossil faunas show an extreme paucity of specimens.

\section{FORAMINIFERA IN GRAB SAMPLES AND IN TOP 2 GENTIMETERS OF CORES}

[Approximate washed volume $10 \mathrm{~cm}^{3}$; depth given in meters below sea level]

Kodiak Harbor, Kodiak Island, depth 10 meters

[No diatoms, Radiolaria, or sponge spicules seen]

$$
\text { Specimens }
$$

Arenaceous benthonic:

Alveolophragmium subglobosum _._._. 1 . 1 _...-

Eggerella scabra
Haplophragmoides canariensis

Calcareous benthonic:

Buccella frigida

cf. $B$. inusitata

Buliminella elegantissima

Elphidium bartletti

clavatum _... 37

orbiculare
sp. (smooth)

Nonion labradoricum

Virgulina cf. V. complanata

Core P-7-61, depth 76 meters. Lat $56^{\circ} 24^{\prime} \mathrm{N}$., long $155^{\circ} 36^{\prime} \mathrm{W}$. [One large (1-cm-long) shrimp and about equal volumes of diatoms and Foraminfera]

Arenaceous benthonic:

Eggerella scabra

Calcareous benthonic:

Bolivina pseudoplicata

Buliminella basicostata

Elphidium magellanicum

Elphidium magellanicum --_-_-_-_--

Epistominella exigua

Nonionella auricula bradyi

Rosalina $\mathrm{sp}$

Virgulina cf. $V$. complanata Calcareous planktonic:

Globigerina bulloides Specimens

Total Stained

Core P-9-61, depth 121 meters. Lat $54^{\circ} 55^{\prime} \mathrm{N}$., long $157^{\circ} 59^{\prime} \mathrm{W}$.

Arenaceous benthonic:

Pseudogaudryina atlantica sp benthonic:

Calcareous benthonic:

Angulogerina angulosa

Bolivina decussata -

Cassidulina crassa teretis

tortuosa

Cibicides lobatulus refulgens

Elphidium bartletti cf. E. crispum

Epistominella exigua Lagena spp _. Loxostomum amygdaliformis _...-_._-_.

Nonion scaphum

Pseudopolymorphina lingua

Uvigerina cushmani

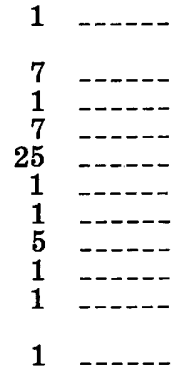
Specimens Total Stained

2 1 21 $2----\cdots$ $-\cdots--$ $-\cdots--$ $-----$ $-\cdots-$ ----$----$ -...-- - - $----$ 
Core P-9-61, depth 121 meters. Lat $54^{\circ} 55^{\prime}$ N., long $157^{\circ} 59^{\prime} \mathrm{W}$.-Continued

Calcareous planktonic:

\begin{tabular}{cc}
\multicolumn{2}{c}{ Specimens } \\
\hline Total & Stained \\
21 & ----- \\
15 & ---- \\
1 & -----
\end{tabular}

pachyderma

Core P-16-61, depth 2,410 meters. Lat $53^{\circ} 53^{\prime}$ N.,long $161^{\circ} 40^{\prime} \mathrm{W}$.

[Diatoms 98 percent; Radiolaria, sponge spicules, Foraminfera compose the remainder, but none very abundant]

Arenaceous benthonic:

$\frac{\text { Specimens }}{\text { Total Stained }}$

Alveolophragmium subglobosum -..--..--

Eggerella bradyi

Trochammina globigeriniformis grisea - gen

Calcareous benthonic:

Bulimina of $B$, auriculato (1)

Elphidium incertum

Elphidiella groenlandica

Globobulimina pacifica - -

Hoeglundina elegans

Nonion labradoricum

Nonionella turgida

(1)

Pyrgo sp ---

Uvigerina peregrina

lcareous planktonic:

Globigerina bulloides pachyderma

2

1

1

1

32

1

- 3

1

$4 \quad--1$

1 - - - -

3

7

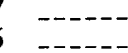

Core P-19-61, depth 4,430 meters. Lat $52^{\circ} 41^{\prime}$ N., long $155^{\circ} 36^{\prime}$ W.

[Diatoms extremely abundant, a few Radiolaria and Foraminifera] Specimens

Arenaceous benthonic:

$\frac{\text { Specimens }}{\text { Total Stained }}$

Adercotryma glomeratum

Alveolophragmium nitidum cf. $A$. nitidum ringens -.-.-subglobosum _... weisneri

Ammobaculites filaformis (smooth)

Ammomarginulina foliacea

Cystammina galeata

Eggerella bradyi

Glomospira gordialis

Haplophragmoides canariensis _.......... nitida

Hyperammina cf. $\mathrm{H}$ friabilis

Jaculella acuta (all chitinous) -........

Nodellum membranaceum

Oculosiphon cf. O. linearis -

Psammosiphonella sp -

Reophax diffugiformis -

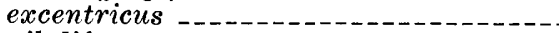
pilulifer

Trochammina globigeriniformis grisea sp. (like chitinous)

Calcareous benthonic:

Cassidulina crassa

Cibicides bradyi

Miliolinella subrotunda

Pullenia subcarinata

Calcareous planktonic:

Globigerina bulloides _._._._._._._._2 corroded

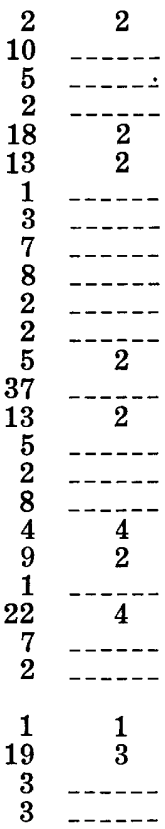

Core P-10-61, depth 4,170 meters. Lat $54^{\circ} 51^{\prime}$ N., long $155^{\circ} 24 \mathrm{~W}$. [Diatoms and Radiolaria abundant]

Arenaceous benthonic:

Specimens

Total Stained

Alveolophragmium subglobosum nitidum

Ammomarginulina foliacea

Cyclammina trullissata

2

3

1

1
Core P-10-61, depth 4,170 meters. Lat $54^{\circ} 51^{\prime}$ N., long $155^{\circ} 24^{\prime} \mathrm{W}$.-Continued

Specimens

Total Stained

Arenaceous benthonic-Continued

Cystammina galeata

Spiroplectammina biformis _..._... 2 -..-

Trochammina globigeriniformis -

Calcareous benthonic:

Cassidulina ef. C. subglobosa _-_._-_- 1

Core P-12-61, depth 6,560 meters. Lat $53^{\circ} 16^{\prime}$ N., long $161^{\circ} 33^{\prime} \mathbf{W}$.

Arenaceous benthonic:

Specimens

Adercotryma glomeratum

Alveolophragmium nitidum cf. $A$. nitidum

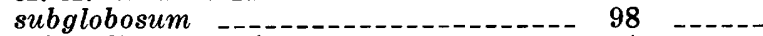

Ammobaculites americanus _...-.-.-.-.- 1 ..... agglutinans filaformis (smooth) agglutinans filaformis (rough) _..... 29 _....-

Astrorhiza _-_._-_-___._Fragments sp. A _....

Jaculella acuta

Psammosiphonella sp -

Reophax dentalinaformis _.......-. 2 -...diffugiformis .... 39 nodulosus -... 22 scotti (chitinous) _... 1 -

Rhizammina $\mathrm{sp}$
Trochammina globigeriniformis

Trochammina sp. (like chitinous) -

Trochammina sp. (chitinous)

Calcareous benthonic:

Eponides? sp -

Involutina tenuis -

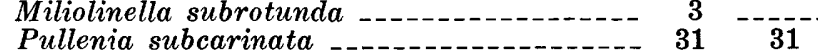

Core $P-48-61$, depth 4,650 meters. Lat $51^{\circ} 41^{\prime}$ N., long $161^{\circ} 07^{\prime} \mathrm{W}$.

[Diatoms extremely abundant (<99 percent), Radiolaria and Foraminifera

Arenaceous benthonic:

Total specimens

Adercotryma glomeratum

Alveolophragmium nitidum 21 cf. $A$. nitidum

scitulum -..- 3 subglobosum

weisneri -.......

Ammobaculites americanus agglutinans filaformis (smooth) agglutinans filaformis (rough)

Ammomarginulina foliacea

Astrorhiza

Baculogypsina or Thurammina

Cornuspira incerta

Cyclammina trullissata

Cystammina galeata

Eggerella bradyi scabra

Glomospira gordialis

Hormosina -..--_-_-_-_-_-_-_-_-_-_._Fragments

Hyperammina cylindrica

Jaculella acuta

Reophax diffugiformis

dentalinaformis distans

scorpiurus

65

Rhabdammina

Saccammina sphaerica _-___._._-_._-_. 19

Spiroplectammina biformis

Trochammina globigeriniformis _..._..._ 28 grisea inflata nitida

1
10

Calcareous benthonic:

Nonion? sp. chitinous 
Core P-49-61, depth 5,000 meters. Lat 59 $50^{\prime}$ N., long $160^{\circ} 57^{\prime} \mathrm{W}$. [Diatoms <95 percent, Radiolaria 4 percent, Foraminifera and sponge skeletons compose the rest] Specimens

Arenaceous benthonic:

Adercotryma glomeratum

Alveolophragmium cf. A. nitidum ringens subglobosum _.......

Ammobaculites agglutinans filaformis (rough)

Baculogypsina or Thurammina

Cyclammina cancellata trullissata

Dorothia exilis

Globotextularia anceps

Glomospira gordialis

Hormosina globulifera _..._. Hyperammina cylindrica

Involutina tenuis

Jaculella acuta

Placopsilina bradyi

Psammosiphonella sp

Reophax difflugiformis

Spiroplectammina biformis

Trochammina globigeriniformis _....... 35 nana nitida chitinous

Calcareous benthonic:

Cibicides bradyi

Cibicidoides mundulus

Ehrenbergina hystrix

Epistominella exigua umbonifera

Gyroidina lamarckiana

Hoeglundina elegans

Miliolinella subrotunda

Melonis affine

Nonion sp chitinous

Calcareous planktonic:

Globigerina bulloides _._._. 3 corroded

Core P-52-61, depth 5,160 meters.Lat $43^{\circ} 47^{\prime} \mathrm{N}_{\text {., }}$ long $160^{\circ} 36^{\prime} \mathrm{W}$.

[Diatoms $50 \pm$ percent, Radiolaria $50 \pm$ percent, a few Foraminifera and sponge skeletons]

Arenaceous benthonic:

Adercotryma glomeratum

Alveolophragmium of $A$ nitidum

Ammobaculites agglutinans

Dorothia exilis

Eggerella scabra

Glomospira gordialis

Placopsilina bradyi

Psammosiphonella sp

Spiroplectammina biformis .

Reophax diffugiformis _.............

Trochammina globigeriniformis grisea nitida Specimens

Total Stained

alcareous benthonic:

Cibicides bradyi

Epistominella exigua

Miliolinella subrotunda

Ophthalmidium acutimargo

Pullenia subcarinata

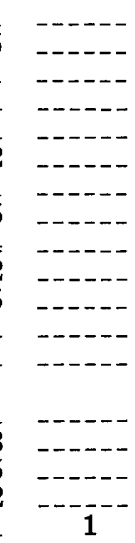

Core P-56-61, depth 5,400 meters, Lat $39^{\circ} 20^{\prime} \mathrm{N}$,, long $160^{\circ} 24^{\prime} \mathrm{W}$.

[Radiolaria 95 percent, diatoms 4 percent, Foraminifera and sponge skeletons 1 percent]

Arenaceous benthonic:

Total Stained

Adercotryma glomeratum

Alveolophragmium nitidum

Ammobaculites americanus

1

6

1
Core P-56-61, depth 5,400 meters. Lat $39^{\circ} 20^{\prime} \mathrm{N}$., long $160^{\circ} 24^{\prime}$ W.-Continued

Specimens

Total Stained

Arenaceous benthonic-Continued

Cornuspira involvens

Glomospira gordialis _.......

Hyperammina cylindrica

Marsipella cylindrica

Psammosiphonella

Reophax dentalinaformis .............

Trochammina globigeriniformis _...-

Calcareous benthonic:

Cibicides bradyi

Discorbis cf. $D$. rost

Planktonic benthonic:

Candeina nitida

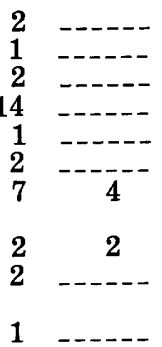

Core P-64-61, depth 5,830 meters. Lat $30^{\circ} 16^{\prime}$ N., long $160^{\circ} 07^{\prime} \mathrm{W}$.

[A few Radiolaria, sponge skeletons, Foraminifera]

Total specimens

Arenaceous benthonic:

$\begin{array}{ll}\text { Aschemonella sp } & 3 \\ \text { Bigenerina minutissima } & \end{array}$

$\begin{array}{ll}\text { Bigenerina minutissima } & 1 \\ \text { Reophax diffugiformis } & \end{array}$

Rhabdammina sp

Saccammina sphaerica 3

Trochammina globigeriniformis _. 1

Core P-69-61, depth 4,820 meters. Lat $23^{\circ} 30^{\prime}$ N., long $159^{\circ} 58^{\prime} \mathrm{W}$. [A few Radiolaria, sponge skeletons, Foraminifera]

Specimens

Arenaceous benthonic:

Alveolophragmium subglobosum

Ammobaculites agglutinans _......-

Cyclammina sp

Glomospira gordialis

Reophax diffugiformis scorpiurus

Rhabdammina sp

Trochammina globigeriniformis _.....nitida Total Stained

Eponides bradyi

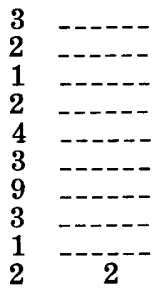

Core P-13-61, depth 7,000 meters. Lat $51^{\circ} 28^{\prime} \mathrm{N}$., long $168^{\circ} 38^{\prime} \mathrm{W}$. [Diatoms 98 percent, Radiolaria $<2$ percent, Foraminifera $<1$ percent]

Specimens

Arenaceous benthonic:

Adercotryma glomeratum

Alveolophragmium nitidum scitulum weisneri

Weisnerta

Eggerella scabra

Hyperammina cylindrica ...............

Marispella cylindrica

Reophax dentalinaformis diffugiformis excentricus nodulosus

scorpiurus -

Rhabdammina sp -

Spiroplectamina biformis

Trochammina globigeriniformis -.....-

Trochammina (chitinous) _.........

Calcareous benthonic:

Pullenia subcarinata

Core P-41-61, depth 7,230 meters.

Lat $50^{\circ} 24^{\prime}$ N., long $176^{\circ} 30^{\prime}$ W., Aleutian Trench.

[Diatoms about 90 percent; Foraminifera 5 percent; sponge spicules and skeletons and Radiolaria make up 5 percent] Specimens

Arenaceos benthonic:

Total Stained

Adercotryma glomeratum

Alveolophragmium c.f. A. nititum scitulum -....... subglobosum

12 
Core P-41-61, depth 7,230 meters. Lat $50^{\circ} 24^{\prime} \mathrm{N}$., long $176^{\circ} 30^{\prime}$ W., Aleutian Trench-Continued

Arenaceous benthonic-Continued Ammobaculites agglutinans filaformis

(smooth)

(rough) -

Ammoscalaria tenuimargo -

Astrorhiza sp

Baculogypsina? sp

Hyperammina friabilis -

Involutina tenuis

Jaculella acuta

Placopsilina brady $\bar{i}$

Reophax difflugiformis ................ 79 excentricus - 103 nodulosus

Saccammina sphaerica

Spiroplectammina biformis -

Trochammina charlottensis _........ 4 -.... globigeriniformis -

Trochammina (chitinous) -

Calcareous benthonic:

Pullenia subcarinata

$8 \quad 8$

Core P-40-61, depth 5,500 meters. Lat $48^{\circ} 06^{\prime}$ N.,long $176^{\circ} 32^{\prime} \mathrm{W}$.

[Diatoms about 85 percent; Foraminifera 5 percent; Radiolaria, sponge skeletons, and fish remains make up 10 percent]

Specimens

Arenaceous benthonic:

Adercotryma glomeratum

Alveolophragmium nitidum subglobosum

Ammobaculites aggìtinans filaformis

(smooth)

Bigenerina minutissima -

Cyclammina cancellata trullissata

Cystammina galeata

Eggerella bradyi

Glomospira gordialis

Hormosina globulifera -...-.-.-1 $1+$ fragments

Jaculella acuta -.-

Miliolinella subrotunda

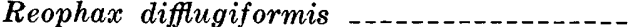
nodulosus scorpiurus

Trochammina globigeriniformis nitida

Calcareous benthonic:

Bolivina robusta

Total Stained

Core P-38-61, depth 5,610 meters. Lat $45^{\circ} 50^{\prime}$ N., long $176^{\circ} 47^{\prime} \mathrm{W}$.

[Formaminifera about 5 percent; diatoms 75 percent; Radiolaria 15 percent; sponge skeletons and spicules and fish teeth make up 5 percent]

Specimens

Arenaceous benthonic:

Adercotryma glomeratum

Alveolophragmium nitidum cf. A. nitidum

subglobosum mobaculites filaformis (smooth) -..-americanus?

Ammoscalaria tenuimargo

Astrorhiza sp

Bigenerina minutissima

Cyclammina cancellata

trullissata

Eggerella fusca

Glomospira gordialis - 15

Jaculella acuta

Placopsilina bradyi

Psammosiphonella sp
Total Stained

8

(----

9 -

-.---

$----$

$-----$

--- -

$----$

- - - -

$----$

$----$

-.--

$----$

$-----$
- - - -
Core P-38-61, depth 5,610 meters. Lat $45^{\circ} 50^{\prime}$ N., long $176^{\circ} 47^{\prime} \mathrm{W}$.- Continued

\section{Specimens}

Total Stained

Arenaceous benthonic-Continued

Reophax difflugiformis

distans

excentricus

nodulosus

Spiroplectammina biformis

Tritaxis cf. T. conica

Trochammina globigeriniformis

nitida

14

$12--\frac{-}{6}$

11

13

8 -....

29 --.--

33 -..--

-

$-----$

Calcareous benthonic:

Miliolinella subrotunda

Nonion sp -.-..-

Quinqueloculina sp

1

Core P-35-61, depth 5,530 meters. Lat $41^{\circ} 19^{\prime}$ N., long $177^{\circ} 02^{\prime} \mathrm{W}$.

[Radiolaria about 90 percent, diatoms 7 percent, sponge and fish remains 3 percent, Foraminifera $<1$ percent]

Calcareous benthonic:

Miliolinella subrotunda

Total specimens

Ophthalmidium pusillum

Core P-33-61, depth 5,230 meters. Lat $39^{\circ} 15^{\prime}$ N., long $176^{\circ} 56^{\prime} \mathrm{W}$.

[Radiolaria about 90 percent, diatoms 5 percent, Foraminifera 3 percent, sponge and fish remains 2 percent] Specimens

Arenaceous benthonic:

Alveolophragmium nitidum subglobosum wiesneri flaformis americanus -...-......-.

(rough) -

Ammomarginulina foliacea Astrorhiza sp Eggerella advena

Glomospira gordialis

Hormosina normani

Psammosiphonella sp

Psammosphaera fusca $-0---0$ phax scorpiurus

Rhabdammina sp

Trochammina grisea nitida

Calcareous benthonic:
Miliolinella subrotunda

Total Stained

Ophthalmidium pusillum

$---1$

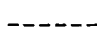

-----

- - - -

$-----$

- - - -

- - - - -

- - - -

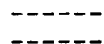

$---\frac{-1}{2}$

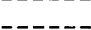

-----

e $-32-61$, depth 5,400 meters. Lat $36^{\circ} 50^{\prime}$ N.,long $177^{\circ} 30^{\prime} \mathrm{W}$.

[Radiolaria 90 percent, diatoms 6 percent, Foraminifera 1 percent, sponge and fish remains 3 percent]

Specimens

Arenaceous benthonic:

Alveolophragmium nitidum --1.--

cf. A. nitidum -

ringens --subglobosum

Ammobaculites filaformis (smooth) -..

Ammoscalaria tenuimargo

Bigenerina minutissima _...........-

Cyclammina trullissata

Eggerella advena

Glomospira gordialis _...............

Hormosina globulifera

Hyperammina cylindrica

Psammosiphonella sp

Reophax diffugiformis _........... scorpiurus

Trochammina globigeriniformis grisea nitida 
Core P-32-61, depth 5,400 meters. Lat $36^{\circ} 50^{\prime} \mathrm{N}$., long $177^{\circ} 30^{\prime} \mathrm{W}$.- Continued

\section{Specimens Total Stained}

Calcareous benthonic:

Miliolinella circularis _-_- 9

Ophthalmidium acutimargo

Pullenia subcarinata

20

Core P-29-61, depth 4,810 meters. Lat $32^{\circ} 22^{\prime} \mathrm{N}$., long $177^{\circ} 20^{\prime} \mathrm{W}$. [Radiolaria about 70 percent, diatoms 25 percent, Foraminifera 1 percent, sponges and fish remains 4 percent] Specimens

Arenaceous benthonic:

Ammobaculites fla formis Total Stained

Ammoscalaria tenuimarg

$-----$

-

galeata

1 - 1 - 1

Glomospira gordialis _..._._.

Hormosina globulifera

Hyperammina cylindrica _._.

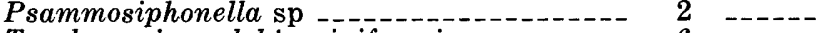

$\begin{array}{lll}\text { Trochammina globigeriniformis } & -\ldots \ldots \\ \text { Trochammina cf. T. nitida }\end{array}$

Calcareous benthonic:

Cassidulina subglobosa

Cibicides bradyi

Hoeglundina elegans

Melonis pompilioides _.............

Spiroloculina sp

Core P-27-61, depth 5,290 meters. Lat $30^{\circ} 06^{\prime} \mathrm{N}$., long $177^{\circ} 30^{\prime} \mathrm{W}$.

[Radiolaria about 85 percent, diatoms 10 percent, sponge or fish remains 5 percent, Foraminifera $<1$ percent] Specimens

Arenaceous benthonic: Total Stained

Alveolophragmium subglobosum

Ammobaculites americanus

Cyclammina trullissata

Cystammina galeata

Glomospira gordialis

Psammosiphonella sp

Calcareous benthonic:

Miliolinella subrotunda

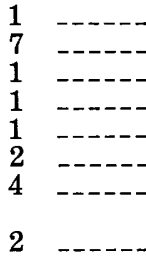

Core P-26-61, depth 5,210 meters. Lat $25^{\circ} 55^{\prime} \mathrm{N}$., long $177^{\circ} 34^{\prime} \mathrm{W}$. [Only a few specimens of Radiolaria, sponge skeletons, fish teeth,
Foraminifera; all present in nearly equal amounts]

Total specimens

Arenaceous benthonic:

Rhabdammina sp _._.

Astrorhiza sp

Glomospira gordialis

Reophax diffugiformis 3

Core P-25-61, depth 5,120 meters. Lat $23^{\circ} 22^{\prime} \mathrm{N}$., long $177^{\circ} 54^{\prime} \mathrm{W}$.

[No Radiolaria, diatoms, or sponges. few fish teeth, Foraminifera.

Northernmost sample with planktonic Foraminifera]

Specimens

Arenaceous benthonic:

Total Stained

Reophax difflugiform

Rhabdammina sp

Calcareous planktonic:

Globigerina bulloides

2 inflata

1

1

\section{FORAMINIFERA BELOW TOP 2 CENTIMETERS OF CORES}

[ Core depths given in meters below sea level. Column headings are depths below sea floor in centimeters. Data indicate number of specimens, $X$ indicates presence of fragments

Core $\mathrm{P}-10-61$, depth 4,170 meters

Barren
Core P-12-61, depth 6,560 meters

Alveolophragmium subglobosum _..--

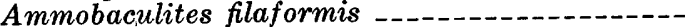

Reophax dentalinaformis

$10-11 \quad 20-21$

3

Core P-13-61, depth 7,000 meters

$10-11 \quad 20-21 \quad 30-31$

Alveolophragmium subglobosum

1

Core P-16-61, depth 2,410 meters

Bulimina affinis

$10-11 \quad 20-21 \quad 30-31 \quad 40-41$

Elphidium clavatum

Uvigerina peregrina

?Eponides sp

1
1
1

Nonion

labradoricum

Lagenid spp

Core P-19-61, depth 4,430 meters

Cystammina galeat

Jaculella acuta

Placopsilina confusa

-

Alveolophragmium subglobosum _.....-

Adercotryma glomeratum

Eggerella bradyi

Trochammina nitida

Hyperammina fragments

Cyclammina cancellata

Ammobaculites cf $A$ americanus

Miliolinella fragments

Trochammina

\section{Core P-25-61, depth 5,120 meters}

Globigerinita

voluta

Globotruncana

sp --:--

$\begin{array}{llllllll}10-11 & 20-21 & 30-31 & 40-41 & 50-51 & 60-61 & 70-71 & 80-81\end{array}$

globulosa

Colomia sp

Guembelina

$$
\text { costulata }
$$

Globotruncana

marginata

Core P-32-61, depth 5,400 meters

$$
10-11 \quad 20-21 \quad 30-31 \quad 40-41 \quad 50-51 \quad 60-61
$$

Eggerella bradyi_.._ _-

Miliolinella

subrotunda

Core P-33-61, depth 5,230 meters

$10-11 \quad 20-21 \quad 30-31 \quad 40-41 \quad 50-51$

Reophax fragments _...- _.. $\times$-..-

Core P-38-61, depth 5,610 meters

$\begin{array}{lllllll}10-11 & 20-21 & 30-31 & 40-41 & 50-51 & 60-61 & 70-71\end{array}$

Reophax fragments _- $\times \times \times$... $\times$...

Alveolophragmium

subglobosum _..._ _..-

Miliolinella

subrotundum

Core $P-40-61$, depth 5,500 meters

Alveolophragmium subglobosum --.----

Reophax dentalinaformis _..........

Hyperammina spp

Core P-41-61, depth 7,230 meters

$\begin{array}{ccc}10-11 & 20-21 & 30-31 \\ 1 & 1 & ---- \\ 2 & --\overline{8} & --- \\ --- & 8 & 4\end{array}$

$0-11 \quad 20-21 \quad 30-31 \quad 40-41$

Alveolophragmium subglobosum _. 
Core P-48-61, depth 4,650 meters

Psammosiphonella sp

Hormosina globulifera

Saccammina sp

americanus

Adercotryma glomeratum

Reophax difflugiformis sp subglobosum scitulum nitidum

Trochammina nitida

Cyclammina cancellata

a

Core P-49-61, depth 5,000 meters

\begin{tabular}{|c|c|c|c|c|c|c|}
\hline & $10-11$ & $20-21$ & $s 0-s 1$ & $40-41$ & $50-51$ & $60-61$ \\
\hline $\begin{array}{l}\text { Alveolophragmium } \\
\text { subglobosum } \\
\text { Cyclammina }\end{array}$ & 13 & 2 & 2 & 9 & 4 & 3 \\
\hline cellata & 2 & -- & 1 & 1 & & 2 \\
\hline radui & 3 & --- & --- & --- & $\ldots$ & --- \\
\hline & 1 & $-\ldots$ & --- & --- & --- & --- \\
\hline lon & 1 & ---- & --- & --- & $m$ & - \\
\hline opho & $x$ & $--\overline{2}$ & --- & $-\cdots$ & --- & $\overline{1}$ \\
\hline
\end{tabular}

Core P-56-61, depth 5,400 meters

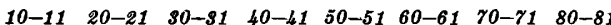

Arenaceous

fragments

Core P-63-61, depth 5,830 meters

$10-1120-21,30-31,40-41,50-51,60-61,70-71$, $80-81,90-91,100-101,120-121$

Hyperammina? sp -.- 1 - 1 -

Core P-64-61, depth 5,830 meters

Barren

$10-11$

Core P-69-61, depth 4,820 meters

Barren $10-11 \quad 20-21 \quad 30-31 \quad 40-41$

\section{SYSTEMATIC CATALOG}

Family ASTRORHIZIDAE Brady, 1881

Genus RHABDAMMINA M. Sars in Carpenter ,1869

Rhabdammina abyssorum M. Sars

Rhabdammina abyssorum M. Sars, 1869, Fordhandl Vidensk.Selsk. Christiania, Aar 1868, p. 248.

Barker, 1960, Soc. Econ. Paleontologists and Mineralogists Spec. Pub 9, p. 42, pl. 21, figs. 1-13.

The species occurs generally as fragments of extremely coarse-grained tubes, sometimes branching. The tubes are coarser grained in the northern samples but are moderately coarse in the southern ones.

Distribution.-Lat $23^{\circ}$ to $51^{\circ} \mathrm{N}$.

\section{Genus RHIZAMMINA, 1879}

Rhizammina? sp .

Plate 1, figure 2

The species occurs as tubular fragments. The walls of the tubes are composed of fine sand held together with abundant cement.

Distribution.-Lat $32^{\circ}$ to $53^{\circ} \mathrm{N}$.

\section{Genus MARSIPELLA Norman, 1878}

Marsipella cylindrica Brady

Plate 1, figure 3

Marsipella cylindrica Brady, 1882, Royal Soc. Edinburgh Proc., v. 11, p. 714.

Brady, 1884, Challenger Repts., Zoology, v. 9, p. 265, 266, pl. 24, figs. 20-22.

Cushman, 1910, U.S. Natl. Mus. Bull. 71, pt. 1, p. 30, figs. 15, 16.

Barker, 1960, Soc. Econ. Paleontologists and Mineralogists Spec. Pub. 9, p. 48, pl. 24, figs. 20-22.

Distribution.-Found at two stations (56 and 13) lat $39^{\circ}$ to $52^{\circ} \mathrm{N}$. Cushman reported it in four North Pacific stations off Hawaii and Japan.

\section{Genus BATHYSIPHON M. Sars in G. Sars, 1872 \\ Bathysiphon discreta (Brady) \\ Plate 1, figure 4}

Rhabdammina discreta Brady, 1881, Micros. Sci. Quart. Jour., new ser., v. 21 , p. 48.

Brady, 1884, Challenger Repts., Zoology, v. 9, p. 268, pl. 22, figs. 7-10.

Cushman, 1910, U.S. Natl. Mus. Bull. 71, pt. 1, p. 27, 28, fig. 13.

Psammosiphonella discreta (Brady). Barker, 1960, Soc. Econ. Paleontologists and Mineralogists Spec. Pub. 9, pl. 22, figs. 7-10.

This species is characterized by its smooth wall texture and light color.

Distribution.-Lat $30^{\circ}$ to $53^{\circ}$ N. A few specimens are found at many stations.

\section{Genus JACULELLA Brady, 1879 \\ Jaculella acuta Brady \\ Plate 1, figure 5}

Jaculella acuta Brady, 1879, Micros. Sci. Quart. Jour., new ser., v. 19 , p. 35 , pl. 3, figs. $12,13$.

Brady, 1884, Challenger Repts., Zoology, v. 9, p. 255, pl. 22, figs. 14-18.

Cushman, 1910, U.S. Natl. Mus. Bull. 71, pt. 1, p. 70 , figs. 90, 91.

Barker, 1960, Soc. Econ. Paleontologists and Mineralogists Spec. Pub. 9, p. 44, pl. 22, figs. 14-18.

?Jaculella acuta Brady. Heron-Allen and Earland, 1934, Discovery Repts., v. 10, Foraminifera, pt. 3, p. 72, 73, pl. 2, figs. 19, 20.

These specimens are identical with Heron-Allen and Earland's illustrations, and the early part of the test is composed of pseudochitin.

Distribution.-Lat. $46^{\circ}$ to $53^{\circ} \mathrm{N}$. Common in the Aleutian Trench area.

\section{Genus HYPERAMMINA Brady, 1878}

Hyperammina spp.

Plate 1, figure 6

Probably several species are present. One group seems to be larger and have a coarser wall (cf. $H$. 
friabilis Brady, 1884, Challenger Repts., Zoology, v. 9, p. 46, pl. 23, figs. $1,2,5,6$ ). Another group is smaller and more finely arenaceous (cf. $H$. cylindrica Parr, 1950, Foraminifera, $B A N Z$ Antarctic Research Exped., 1929-1931, Repts., ser. B, v. 5, pt. 6, p. 254, pl. 3, fig. 5). However, where they occur together, it is so difficult to draw a line between the two groups that they are considered together.

Distribution.-Lat $32^{\circ}$ to $50^{\circ} \mathrm{N}$.

Family SACCAMMINIDAE Brady, 1884 Genus PSAMMOSPHAERA Schulze, 1875

Psammosphaera rustica Heron-Allen and Earland

Psammosphaera rustica Heron-Allen and Earland, 1912, Royal Micros. Soc. London Jour., p. 383, pl. 5, figs. 3, 4 ; pl. 6, figs. 2-4.

Distribution.-Found in only one core sample, $\mathrm{P}$ 33-61 (lat $39^{\circ}$ N., depth 5,230 m).

\section{Genus SACCAMMINA M. Sars in Carpenter, 1869}

\section{Saccammina sphaerica M. Sars}

Saccammina sphaerica M. Sars, 1869, Forhandl. Vidensk.Selsk. Christiania, Aar 1868, p. 248.

Brady, 1884, Challenger Repts., Zoology, v. 9, p. 253, pl. 18, figs. 11-15, 17.

Cushman, 1910, U.S. Natl. Mus. Bull. 71, pt. 1, p. 39, 40, figs. 33-36.

Barker, 1960, Soc. Econ. Paleontologists and Mineralogists Spec. Pub. 9, p. 36, pl. 18, figs. 11-15, 17.

Distribution.-Lat $30^{\circ}$ to $52^{\circ}$ N. Abundant in the Aleutian Trench.

\section{Genus THURAMmINA Brady, 1879 \\ Thurammina papillata Brady}

Plate 1, figure 7

Thurammina papillata Brady, 1879, Micros. Sci. Quart. Jour., new ser., v. 19 , p. 45 , pl. 5, figs. 4-8.

Brady, 1884, Challenger Repts., Zoology, v. 9, p. 321, pl. 36, figs. 7-18.

Cushman, 1910, U.S. Natl. Mus. Bull. 71, pt. 1, p. 58, fig. 66 .

Barker, 1960, Soc. Econ. Paleontologists and Mineralogists Spec. Pub. 9, p. 74, pl. 36, figs. 7-18.

Distribution.-Lat $48^{\circ}$ to $52^{\circ} \mathrm{N}$. Reported previously from the North Pacific in Challenger stations from depths of 3,400 to 4,700 meters.

\section{Family AMMODISCIDAE Reuss, 1862 Genus GLOMOSPIRA Rzehak, 1885 \\ Glomospira gordialis (Jones and Parker) \\ Plate 1, figure 8}

Trochammina squamata var. gordialis Jones and Parker, 1860, Geol. Soc. London Quart. Jour., v. 16, p. 304.

Parker and Jones, 1865, Royal Soc. London Philos. Trans., v. 155 , p. 408 , pl. 15, fig. 32.

Ammodiscus gordialis (Jones and Parker). Brady, 1884, Challenger Repts., Zoology, v. 9, p. 333, pl. 38, figs. 7-9.
Gordiammina gordialis (Jones and Parker). Cushman, 1910, U.S. Natl. Mus. Bull. 71, pt. 1, p. 76, 77, figs. 98-100. Glomospira gordialis (Jones and Parker). Cushman, 1918, U.S. Natl. Mus. Bull. 104, pt. 1, p. 99, pl. 36, figs. 7-9. Barker, 1960, Soc. Econ. Paleontologists and Mineralogists Spec. Pub. 9, p. 78, pl. 38, figs. 7-9.

Distribution.-Lat $23^{\circ}$ to $53^{\circ} \mathrm{N}$. Present, often abundantly, at stations just south of the Aleutian Trench.

\section{Family HORMOSINIDAE Haeckel, 1894 Genus HORMOSINA Brady, 1879 \\ Hormosina globulifera Brady}

Hormosina globulifera Brady, 1879, Micros. Sci. Quart. Jour., new ser., v. 19, p. 60 , pl. 4 , figs. 4,5 .

Brady, 1884, Challenger Repts., Zoology, v. 9, p. 326, pl. 39, figs. 1-6.

Cushman, 1910, U.S. Natl. Mus. Bull. 71, pt. 1, p. 9395, figs. 136, 137.

Barker, 1960, Soc. Econ. Paleontologists and Mineralogists Spec. Pub. 9, p. 80, pl. 39, figs. 1-6.

Distribution.-Lat $32^{\circ}$ to $52^{\circ} \mathrm{N}$. Generally present as fragments recognizable by the texture of the wall. Brady described these as fragments of a peculiarly deep-water organism. It was found at 21 Challenger stations, only five of which had a depth of less than 1,800 meters.

\section{Genus REOPHAX Montfort, 1808 \\ Reophax dentalinaformis Brady \\ Plate 1, figure 9}

Reophax dentalinaformis Brady, 1881, Micros. Sci. Quart. Jour., new ser., v. 21, p. 49.

Brady, 1884, Challenger Repts., Zoology, v. 9, p. 293, pl. 30, figs. 21, 22.

Barker, 1960, Soc. Econ. Paleontologists and Mineralogists Spec. Pub. 9, p. 62, pl. 30, figs. 21, 22.

Distribution.-Occurs from lat $39^{\circ}$ to $51^{\circ} \mathrm{N}$. Brady listed it from 21 stations, only four of which were shallower than 1,800 meters.

\section{Reophax difflugiformis Brady}

Plate 1, figure 10

Reophax diffugiformis Brady, 1879, Micros. Sci. Quart. Jour., new ser., v. 19 , p. 51 , pl. 4 , fig. 3 .

Brady, 1884, Challenger Repts., Zoology, v. 9, p. 289, pl. 30, figs. 1-4.

Barker, 1960, Soc. Econ. Paleontologists and Mineralogists Spec. Pub. 9, p. 62, pl. 30, figs. 1-4.

Proteonina diffugiformis (Brady). Cushman, 1910, U.S. Natl. Mus. Bull. 71, pt. 1, p. 41, 42, figs. 40, 41.

Distribution.-This variable species occurs commonly at nearly all stations, most abundantly in deep water of the Aleutian Trench.

\section{Reophax distans Brady \\ Plate 1, figure 11}

Reophax distans Brady, 1881, Micros. Sci. Quart. Jour., new ser., v. 21, p. 50. 
Brady, 1884, Challenger Repts., Zoology, v. 9, p. 296, pl. 31, figs. 18-22.

Cushman, 1910, U.S. Natl. Mus. Bull. 71, pt. 1, p. 85, 86, fig. 119.

Barker, 1960, Soc. Econ. Paleontologists and Mineralogists Spec. Pub. 9, p. 64, pl. 31, figs. 18-22.

Distribution.-Lat $39^{\circ}$ to $52^{\circ}$ N., rare and fragile. No specimens with more than three chambers found.

\section{Reophax nodulosus Brady}

Plate 1, figure 12

Reophax nodulosa Brady, 1879, Micros. Sci. Quart. Jour., new ser., v. 19 , p. 52 , pl. 4 , figs. $7,8$.

Brady, 1884, Challenger Repts., Zoology, v. 9, p. 294, pl. 31, figs. 1-9.

Reophax nodulosus Brady. Cushman, 1910, U.S. Natl. Mus. Bull. 71, pt. 1, p. 87, 88, fig. 122.

Barker, 1960, Soc. Econ. Paleontologists and Mineralogists Spec. Pub. 9, p. 64, pl. 31, figs. 1-9.

This species is generally large and robust. It shows a great deal of variation in chamber shape, as shown in Brady's (1884) illustrations.

Distribution.-Lat $46^{\circ}$ to $53^{\circ} \mathrm{N}$.

\section{Reophax pilulifer Brady}

Reophax pilulifera Brady, 1884, Challenger Repts., Zoology, v. 9, p. 292, pl. 30, figs. 18-20.

Reoplax pilulifer Brady. Cushman, 1910, U.S. Natl. Mus. Bull. 71 , pt. 1, p. 85, figs. 117, 118 .

Barker, 1960, Soc. Econ. Paleontologists and Mineralogists Spec. Pub. 9, p. 62, pl. 30, figs. 18-20.

This species is very distinctive and somewhat similar to Hormosina globulifera Brady (1884, p. 53), but it has a coarsely arenaceous wall.

Distribution.-Found in core sample P-19-61 (lat $53^{\circ}$ N., depth $4,430 \mathrm{~m}$ ).

\section{Reophax scorpiurus de Montfort}

Plate 1, figure 13

Reophax scorpiurus de Montfort, 1808, Conch. Syst., v. 1, p. 331, 83d genre.

Brady, 1884, Challenger Repts., Zoology, v. 9, p. 291, pl. 30, figs. 12, 14-17.

Barker, 1960, Soc. Econ. Paleontologists and Mineralogists Spec. Pub. 9, p. 62, pl. 30, figs. 12, 14-17.

This is one of the few arenaceous species that shows strong variation in wall texture. In the shoreward samples (P-13-61, P-19-61, P-38-61, P-41$61, \mathrm{P}-48-61, \mathrm{P}-49-61)$ the wall texture is very coarse; the specimens are large, and the test generally has only three chambers. In the seaward samples (P-32-61, P-33-61, P-63-61, P-69-61), the wall is fine grained, and sponge spicules are often incorporated in the test, which generally has five chambers.

Distribution.-Lat $23^{\circ}$ to $51^{\circ} \mathrm{N}$.

\section{Reophax scotti Chaster}

Reophax scotti Chaster, 1892, 1st Rept. Southport Soc. Nat. Sci., 1890-91, p. 57, pl. 1, fig. 1.

Höglund, 1947, Zool. Bidrag Fran Uppsala, v. 26, p. 9496, fig. 72.

Cushman and McCulloch, 1939, Allan Hancock Pacific Exped. Repts., v. 6, no. 1, p. 61, 62, pl. 3, fig. 11.

Distribution.-A single specimen was found in only one core sample $\left(\mathrm{P}-12-61\right.$, lat $53^{\circ} \mathrm{N}$., depth $6,560 \mathrm{~m}$ ). The extreme fragility of the test may account for its scarcity in prepared samples.

\section{Family LITUOLIDAE de Blainville, 1825 Genus ADERCOTRYMA Loeblich and Tappan, 1952 \\ Adercotryma glomerata (Brady) \\ Plate 1, figure 14}

Lituola glomerata Brady, 1878, Ann. Mag. Nat. History, ser. 5, v. 1 , p. 433 , pl. 20 , figs. 1 a-c.

Adercotryma glomeratum (Brady). Loeblich and Tappan, 1952, Washington Acad. Sci. Jour., v. 42, no. 5, p. 141, 142 , figs. 1-4.

Barker, 1960, Soc. Econ. Paleontologists and Mineralogists Spec. Pub. 9, pl. 70, pl. 34, figs. 15-18.

These specimens are somewhat broader and have more deeply depressed sutures than those figured by Brady and by Loeblich and Tappan.

Distribution.-Lat $30^{\circ} \mathrm{N}$. to Aleutian Trench. According to Brady $(1884$, p. 309, 310), this species was found in relatively shallow water in Arctic seas and in deep water (greater than $3,600 \mathrm{~m}$ ) of tropical and subtropical latitudes.

\section{Genus CYClAMMINA Brady, 1879 \\ Cyclammina cancellata Brady \\ Plate 1, figure 18}

Cyclammina cancellata Brady, 1879, Micros. Sci. Quart. Jour., new ser., v. 19, p. 62.

Brady, 1884, Challenger Repts., Zoology, v. 9, p. 351, pl. 37, figs. 8-16.

Cushman, 1910, U.S. Natl. Mus. Bull. 71, pt. 1, p. 110 111, figs. 168-171.

Barker, 1960, Soc. Econ. Paleontologists and Mineralogists Spec. Pub. 9, p. 76, pl. 37, figs. 8-16.

Distribution.-Lat $46^{\circ}$ to $50^{\circ} \mathrm{N}$. Brady listed this species from two samples from the North Pacific, east from Japan, at depths of 3,400 and 5,300 meters.

\section{Cyclammina trullissata (Brady) \\ Plate 1, figure 15}

Trochammina trullissata Brady, 1879, Micros. Sci. Quart. Jour., new ser., v. 19, no. 73 , p. 56, pl. 5, figs. 10 a, b.

Brady, 1884, Challenger Repts., Zoology, v. 9, p. 342, pl. 40, fig. 13 (not fig. 14, 15).

Cyclammina bradyi Cushman, 1910, U.S. Natl. Mus. Bull. 71, pt. 1 , p. 113 , fig. 174 .

Cyclammina trullissata (Brady). Barker, 1960, Soc. Econ. Paleontologists and Mineralogists Spec. Pub. 9, p. 82, pl. 40, fig. 13 . 
Distribution.-Widely distributed (lat $30^{\circ}$ to $55^{\circ}$ N.), rare.

\section{Genus ALVEOLOPHRAGMIUM Shchedrina, 1936}

Alveolophragmium nitidum (Göes)

Plate 1, figure 16

Haplophragmium nitidum Göes, 1896, Harvard Coll. Mus. Comp. Zoology Bull., v. 29, p. 30, pl. 3, figs. 8, 9.

Haplophragmoides nitidum Göes. Cushman, 1920, U.S. Natl. Mus. Bull. 104, pt. 2, p. 44.

Haplophragmoides nitidus (Göes). Cushman, 1920, U.S. Natl. Mus. Bull. 104, pt. 2, p. 44.

Haplophragmoides nitidus (Göes). Heron-Allen and Earland, 1934, Discovery Repts., v. 10, Foraminifera, pt. 3, p. 88, 89, pl. 3, figs. 3-6.

This species is extremely smooth walled, brown in color, completely involute, with 4 or $4 \frac{1}{2}$ chambers visible.

Distribution.-Most common north of lat $50^{\circ} \mathrm{N}$. in the Aleutian Trench area.

\section{Alveolophragmium cf. A. nitidum (Göes) \\ Plate 1, figure 17}

Haplophragmoides nitidus (Göes). Heron-Allen and Earland, 1934, Discovery Repts., v. 10, Foraminifera, pt. 3 , p. 88,89 , last paragraph.

This form differs from the typical one in having a slightly coarser wall and more inflated chambers. It occurs with the typical form.

Distribution.-Most common north of lat $50^{\circ} \mathrm{N}$. Extremely abundant in the deep waters of the Aleutian Trench $(7,000 \mathrm{~m}$ or more).

\section{Alveolophragmium ringens (Brady)}

Trochammina ringens Brady, 1879, Micros. Sci. Quart. Jour., new ser., v. 19, p. 57, pl. 5, fig. 12 .

Brady, 1884, Challenger Repts., Zoology, v. 9, p. 343, pl. 40, figs. 17, 18.

Haplophragmoides ringens (Brady). Cushman, 1910, U.S. Natl, Mus. Bull. 71, pt. 1, p. 109, fig. 166.

Alveolophragmium ringens (Brady). Parker, 1954, Harvard Coll. Mus. Comp. Zoology Bull., v. 111, no. 10, p. 487, pl. 1, fig. 19.

Barker, 1960, Soc. Econ. Paleontologists and Mineralogists Spec. Pub. 9, p. 82, pl. 40, figs. 17, 18.

Specimens are identical with those figured by Brady.

Distribution.-Widely distributed, but rare. Brady (1884, p. 344) did not record it from any Pacific stations.

\section{Alveolophragmium scitulum (Brady) \\ Plate 1, figure 19}

Haplophragmium scitulum Brady, 1881, Micros. Sci. Quart. Jour., new ser., v. 21, p. 50.

Brady, 1884, Challenger Repts., Zoology, v. 9, p. 308, p. 34, figs. 11-13.

Alveolophragmium scitulum (Brady). Parker, 1954, Har- vard Coll. Mus. Comp. Zoology Bull., v. 111, no. 10, p. 487.

Barker, 1960, Soc. Econ. Paleontologists and Mineralogists Spec. Pub. 9, p. 70, pl. 34, figs. 11-13.

Distribution.-Present only in the Aleutian Trench area.

\section{Alveolophragmium subglobosum (G. O. Sars)}

Plate 1, figure 20

Haplophragmium latidorsatum (Bornemann). Brady, 1884, Challenger Repts., Zoology, v. 9, p. 307, pl. 34, figs. 7, 8, 10 .

Haplophragmoides subglobosum (G. O. Sars). Cushman, 1910, U.S. Natl. Mus. Bull. 71, pt. 1, p. 105, 106, figs. 162-164.

Labrospira subglobosa (G. O. Sars). Höglund, 1947, Zool. Bidrag. Fran Uppsala, v. 26, p. 144, 145, pl. 11, fig. 2, text fig. 126.

Alveolophragmium subglobosum (G. O. Sars). Barker, 1960, Soc. Econ. Paleontologists and Mineralogists Spec. Pub. 9, p. 70, pl. 34, figs. 7, 8, 10.

Specimens have less deeply depressed sutures than those of Brady. Coarseness of wall is variable.

Distribution.-Widely distributed, from lat $23^{\circ}$ to $54^{\circ}$ N. Brady (1884, p. 308) found it at nine stations in the North Pacific at depths from 3,600 to 7,300 meters.

\section{Alveolophragmium weisneri (Parr) Plate 2, figure 1}

Trochammina trullissata Brady, 1884, Challenger Repts., Zoology, v. 9, p. 342, pl. 40, figs. 14, 15 (not fig. 13).

Labrospira weisneri Parr, 1950, Foraminifera, BANZ Antarctic Research Exped., 1929-1931, Repts., ser. B, v. 5, pt. 6, p. 272 , pl. 4 , figs. $25,26$.

Labrospira arctica Parker, 1952, Harvard Coll. Mus. Comp. Zoology Bull., v. 106, no. 9, p. 399, pl. 2, figs. 7, 12.

Alveolophragmium weisneri (Parr). Parker, 1954, Harvard Coll. Mus. Comp. Zoology Bull., v. 111, no. 10, p. 488, pl. 1, fig. 23.

Barker, 1960, Soc. Econ. Paleontologists and Mineralogists Spec. Pub. 9, p. 82, pl. 40, figs. 14, 15.

Specimens are identical with those illustrated by Brady.

Distribution.-Lat $39^{\circ}$ to $52^{\circ}$ N., rare.

\section{Alveolophragmium? sp. \\ Plate 2, figure 2}

The form is tiny, slightly evolute; sutures are curving, only slightly depressed; periphery is rounded, only slightly lobed; wall is smooth, red-brown, pseudochitinous; aperture is interio-areal.

This is apparently an arenaceous form that has not developed an agglutinating stage (see also Trochammina cf. T. malovensis, p. ). It is very rare.

Distribution.-Core samples $\mathrm{P}-48-61$ (lat $52^{\circ} \mathrm{N}$., depth 4,650 m) and P-49-61 (lat $50^{\circ}$ N., depth $5,000 \mathrm{~m}$ ). 


\section{Genus AMMOBACULITES Cushman, 1910}

Ammobaculites agglutinans (d'Orbigny)

Plate 2, figure 3

Spirolina agglutinans d'Orbigny, 1846, Foram. Fossiles Wien, p. 137, pl. 7, figs. 10-12.

Haplophragmium agglutinans (d'Orbigny). Brady, 1884, Challenger Repts., Zoology, v. 9, p. 301, pl. 32, figs. 19-21, 24-26.

Ammobaculites agglutinans (d'Orbigny). Cushman, 1910, U.S. Natl. Mus. Bull. 71, pt. 1, p. 115, fig. 176.

Barker, 1960, Soc. Econ. Paleontologists and Mineralogists Spec. Pub. 9, p. 66, pl. 32, figs. 19-21, 24-26.

Walls of these specimens are coarser grained than those illustrated by Brady.

Distribution.-Lat $23^{\circ}$ to $50^{\circ}$ N. Brady (1884, p. $301,775)$ listed it from deep water in the North Pacific.

Ammobaculites agglutinans filaformis Heron-Allen and Earland (smooth form)

Plate 2, figure 4

Haplophragmium agglutinans (d'Orbigny). Brady, 1884, Challenger Repts., Zoology, v. 9, p. 301, pl. 32, fig. 23 (not fig. 22).

Ammobaculites agglutinans filaformis Heron-Allen and Earland, 1934, Discovery Repts., v. 10, Foraminifera, pt. 3, p. 92, 93, pl. 3, fig. 12 (not fig. 11).

Barker, 1960, Soc. Econ. Paleontologists and Mineralogists Spec. Pub. 9, p. 66, pl. 32, fig. 23 (not fig. 22).

This smooth-walled form has low regular chambers, and the wall is brown.

Distribution.-Lat $32^{\circ}$ to $53^{\circ} \mathrm{N}$., most abundant in the Aleutian Trench. Heron-Allen and Earland listed this form from deep water in the Antarctic Ocean.

\section{Ammobaculites agglutinans filaformis Heron-Allen and Earland (rough form) \\ Plate 2, figure 5}

Haplophragmium agglutinans (d'Orbigny). Brady, 1884, Challenger Repts., Zoology, v. 9, p. 301, pl. 32, fig. 22 (not fig. 23).

Ammobaculites agglutinans filaformis Heron-Allen and Earland, 1934, Discovery Repts., v. 10, Foraminifera, pt. 3, p. 92,93 , pl. 3, fig. 11 (not fig. 12).

Barker, 1960, Soc. Econ. Paleontologists and Mineralogists Spec. Pub. 9, p. 66, pl. 32, fig. 22 (not fig. 23).

The wall is so coarsely arenaceous that chamber arrangement is difficult to see.

Distribution.-Lat $39^{\circ}$ to $53^{\circ}$ N. This form commonly occurs in the same samples as the smoothwalled form.

\section{Ammobaculites americanus Cushman}

Haplophragmium fontinense Terquem. Brady, 1884, Challenger Repts., Zoology, v. 9, p. 305, pl. 34, figs. 1-4.

Ammobaculites americanus Cushman, 1910, U.S. Natl. Mus. Bull. 71, pt. 1, p. 117, 118, figs. 184, 185.

Barker, 1960, Soc. Econ. Paleontologists and Mineralogists Spec. Pub. 9, p. 70, pl. 34, figs. 1-4.
These specimens are similar to those illustrated by Brady and by Cushman but are thicker.

Distribution.-Lat $30^{\circ}$ to $56^{\circ} \mathrm{N}$., most abundant north of lat $50^{\circ} \mathrm{N}$.

\section{Genus AMMOMARGINULINA Weisner, 1931 \\ Ammomarginulina foliacea (Brady) \\ Plate 2, figure 6}

Haplophragmium foliaceum Brady, 1881, Micros. Sci. Quart. Jour., new ser., v. 21, p. 50.

Brady, 1884, Challenger Repts., Zoology, v. 9, p. 304, 305 , pl. 33, figs. 20-25.

Ammomarginulina foliaceus (Brady). Cushman, 1933, Cushman Lab. Foram. Research Spec. Pub. 4, pl. 10, fig. 6.

Barker, 1960, Soc. Econ. Paleontologists and Mineralogists Spec. Pub. 9, p. 68, pl. 33, figs. 20-25.

Specimens are identical with Brady's illustrations.

Distribution.-Widely distributed (lat $39^{\circ}$ to $55^{\circ}$ N.) but rare. Brady $(1884$, p. 305) found only a few specimens in the North Pacific.

\section{Genus PLACOPSILINA d'Orbigny, 1850 \\ Placopsilina confusa Cushman}

Placopsilina confusa Cushman, 1920, U.S. Natl. Mus. Bull. 104 , pt. 2 , p. 71 , pl. 14 , fig. 16 .

Heron-Allen and Earland, 1934, Discovery Repts., v. 10, Foraminifera, pt. 3, p. 94, 95.

This tiny form occurs abundantly at several localities. It is commonly attached to diatom frustules. Its small size and distinctive reddish-brown color distinguish it from $P$. bradyi Cushman and McCulloch $(1939$, p. 112).

Distribution.-Lat $44^{\circ}$ to $50^{\circ} \mathrm{N}$.

\section{Family TEXTULARIIDAE Ehrenberg, 1838 \\ Genus SPIROPLECTAMMINA Cushman, 1927 \\ Spiroplectammina biformis (Parker and Jones) \\ Plate 2, figure 7}

Textularia agglutinans var. biformis Parker and Jones, 1865 Royal Soc. London Philos. Trans., v. 155, p. 370, pl. 15, figs. 23, 24.

Spiroplecta biformis (Parker and Jones). Brady, 1884, Challenger Repts., Zoology, v. 9, p. 376, pl. 45, figs. 25-27.

Spiroplectammina biformis (Parker and Jones). Cushman, 1927, Cushman Lab. Foram. Research Contr., v. 3, pt. 1, p. 23.

Barker, 1960, Soc. Econ. Paleontologists and Mineralogists Spec. Pub. 9, p. 92, pl. 45, figs. 25-27.

The test is extremely small, fine grained, and brown.

Distribution.-Lat $46^{\circ}$ to $54^{\circ} \mathrm{N}$.

\section{Genus BIGENERINA d'Orbigny, 1820 \\ Bigenerina minutissima Earland Plate 2, figure 8}

Bigenerina minutissima Earland, 1933, Discovery Repts., v. 7, Foraminifera, pt. 2, p. 98, pl. 3, figs. 36-38. 
Heron-Allen and Earland, 1934, Discovery Repts., v. 10, Foraminifera, pt. 3, p. 117, pl. 4, fig. 48.

Distribution.-Lat. $30^{\circ}$ to $48^{\circ}$ N., rare. HeronAllen and Earland rarely found the species in deep waters of the Scotia Sea (lat $55^{\circ}$ to $60^{\circ} \mathrm{S}$.).

Family TROCHAMMINIDAE Schwager, 1877 Genus TROCHAMMINA Parker and Jones, 1859

Trochammina grisea Heron-Allen and Earland

Plate 2, figure 9

Trochammina grisea Heron-Allen and Earland, 1934, Discovery Repts., v. 10, Foraminifera, pt. 3, p. 100, 101, pl. 3, figs. 35-37.

Distribution.-Rare in several stations north of lat $47^{\circ} \mathrm{N}$.

\section{Trochammina inflata (Montagu)}

Plate 2, figure 10

Trochammina inflata (Montagu). Brady, 1884, Challenger Repts., Zoology, v. 9, p. 338, pl. 41, fig. 4.

Cushman, 1910, U.S. Natl, Mus. Bull. 71 , pt. 1, p. 121, 122, fig. 188.

Barker, 1960, Soc. Econ. Paleontologists and Mineralogists Spec. Pub. 9, p. 84, pl. 41, fig. 4.

Distribution.-Specimens identical with those figured by Brady occur in two core samples, P-3861 and $\mathrm{P}-48-61$ (lat $46^{\circ}$ and $52^{\circ} \mathrm{N}$.).

\section{Trochammina kellettae Thalmann}

Plate 2, figure 12

Trochammina peruviana Cushman and Kellett, 1929, U.S. Natl. Mus. Proc., v. 75, art. 25, p. 4, pl. 1, fig. 8.

Trochammina kellettae Thalmann, 1932, Eclogae Geol. Helvetiae, v. 25, p. 313.

Distribution.-A few specimens found at one locality in the Aleutian Trench (core sample P-41-61, lat $50^{\circ} \mathrm{N}$., depth $7,230 \mathrm{~m}$ ).

\section{Trochammina malovensis Heron-Allen and Earland}

Plate 2, figure 13

Trochammina malovensis Heron-Allen and Earland, 1932, Discovery Repts., v. 4, Foraminifera, pt. 1, p. 345, pl. 17, figs. 14-19.

?Haplophragmium turbinatum var. helicoideum Göes, 1896, Harvard Coll. Mus. Comp. Zoology Bull., v. 29, no. 1, p. 30,31 , pl. 3, figs. 10-13.

This species is identical with that in Heron-Allen and Earland's illustrations. The specimen from core sample $\mathrm{P}-12-61$ appears to grade into the pseudochitinous T. cf. T. malovensis (below).

Distribution.-Present at two stations in the Aleutian Trench.

\section{Trochammina cf. T. malovensis Heron-Allen and Earland}

Plate 2, figure 11

This species appears identical with $T$. malovensis except in wall character and in its slightly more irregular shape. The wall is composed of reddishbrown pseudochitin. However, the last few chambers appear finely arenaceous in specimens from core sample P-12-61.

Distribution.-Found abundantly in the Aleutian Trench, rarely as far south as lat $33^{\circ} \mathrm{N}$.

Trochammina nana (Brady)

Plate 2, figure 15

Haplophragmium nanum Brady, 1881, Micros. Sci. Quart. Jour., new ser., v. 21, p. 50.

Brady, 1884, Challenger Repts., Zoology, v. 9, p. 311, pl. 35, figs. 6-8.

Cushman, 1910, U.S. Natl. Mus. Bull. 71, pt. 1, p. 123, figs. 190-192.

Trochammina nana (Brady). Barker, 1960, Soc. Econ. Paleontologists and Mineralogists Spec. Pub. 9, p. 72, pl. 35 , figs. $6-8$.

This small species is variable in number of chambers. It is characteristically dark reddish-brown.

Distribution.-Abundant in core sample P-49-61 (lat $50^{\circ} \mathrm{N}$., depth $5,000 \mathrm{~m}$ ).

\section{Trochammina nitida Brady}

Plate 2, figure 14

Trochammina nitida Brady, 1881, Micros. Sci. Quart. Jour., new ser., v. 21, p. 52.

Brady, 1884, Challenger Repts., Zoology, v. 9, p. 339, pl. 41, figs. 5,6 .

Barker, 1960, Soc. Econ. Paleontologists and Mineralogists Spac. Pub. 9, p. 84, pl. 41, figs. 5, 6.

Distribution.-Lat $24^{\circ}$ to $52^{\circ} \mathrm{N}$. This little species is widely distributed but rare. All but one of the occurrences described by Brady are shallower than 390 meters.

\section{Trochammina globigeriniformis (Parker and Jones) Plate 3, figure 1}

Lituola nautiloidea globigeriniformis Parker and Jones, 1865, Royal Soc. London Philos. Trans., v. 155, p. 407, pl. 15, figs. $46,47$.

Haplophragmium globigeriniforme (Parker and Jones). Brady, 1884, Challenger Repts., Zoology, v. 9, p. 312, pl. 35, figs. $10,11$.

Ammoglobigerina bulloides Eimer and Fickert, 1899, Zeitschr. Wiss. Zoologie, Leipzig, v. 65, pt. 4, p. 107.

Trochammina globigeriniformis (Parker and Jones). Cushman, 1910, U.S. Natl. Mus. Bull. 71, pt. 1, p. 124, 125, figs. 193-195.

Ammoglobigerina globigeriniformis (Parker and Jones). Barker, 1960, Soc. Econ. Paleontologists and Mineralogists Spec. Pub. 9, p. 72, pl. 35, figs. 10, 11.

Distribution.-Lat $23^{\circ}$ to $54^{\circ} \mathrm{N}$. One of the most widely distributed forms. Brady (1884, p. 313) listed it from six stations in the North Pacific, all but one at depths greater than 3,300 meters. Cushman recorded it as one of the most common deep-water species. 


\section{Genus CYSTAMMINA Neumayr, 1889 \\ Cystammina galeata (Brady)}

Plate 3, figure 2

Trochammina galeata Brady, 1881, Micros. Sci. Quart. Jour., new ser., v. 21, p. 52.

Brady, 1884, Challenger Repts., Zoology, v. 9, p. 344, pl. 40, figs. 19-23.

Ammochilostoma galeata (Brady). Cushman, 1910, U.S. Natl. Mus. Bull. 71, pt. 1, p. 127, 128, figs. 198-201.

Cystammina galeata (Brady). Barker, 1960, Soc. Econ. Paleontologists and Mineralogists Spec. Pub. 9, p. 82, pl. 40, figs. 19-23.

Distribution.-Lat $30^{\circ}$ to $55^{\circ}$ N., rare. Brady noted it as rare and found only at great depth in midocean.

\section{Genus TRITAXIS Schubert, 1921}

Tritaxis conica (Parker and Jones)

Valvulina triangularis var. conica Parker and Jones, 1865, Royal Soc. London Philos. Trans., v. 155, p. 406, pl. 15, fig. 27.

Valvulina conica Parker and Jones. Brady, 1884, Challenger Repts., Zoology, v. 9, p. 392, pl, 49, figs. 15, 16.

Tritaxis conica (Parker and Jones). Barker, 1960, Soc. Econ. Paleontologists and Mineralogists Spec. Pub. 9 , p. 100 , pl. 49 , figs. 15,16 .

Distribution.-Lat $35^{\circ} \mathrm{N}$.

\section{Family ATAXOPHRAGMIIDAE Schwager, 1877}

\section{Genus GLOBOTEXTULARIA Eimer and Fickert, 1899}

Globotextularia anceps (Brady)

Haplophragmium anceps Brady, 1884, Challenger Repts.. Zoology, v. 9, p. 313, pl. 35, figs. 12-15.

Globotextularia anceps (Brady). Eimer and Fickert, 1889, Zeitschr. Wiss. Zool., v. 65, p. 679.

Cushman, 1910, U.S. Natl. Mus. Bull. 71, pt. 1, p. 125, 126, fig. 196.

Barker, 1960, Soc. Econ. Paleontologists and Mineralogists Spec. Pub. 9, p. 72, pl. 35, figs. 12-15.

Distribution.-A few specimens found in core samples P-41-61 and P-49-61 (lat 50 N.). Brady noted it in samples from deep water of the Atlantic but not of the Pacific.

\section{Genus DOROTHIA Plummer, 1931 \\ Dorothia exilis Cushman \\ Plate 3, figure 3}

Gaudryina filaformis Berthelin. Brady, 1884, Challenger Repts., Zoology, v. 9, p. 380, pl. 46, fig. 12 .

Dorothia exilis Cushman, 1936, Cushman Lab. Foram. Research Spec. Pub. 6, p. 30.

Barker, 1960, Soc. Econ. Paleontologists and Mineralogists Spec. Pub. 9, p. 94, pl. 46, fig. 12.

These extremely minute forms are probably assignable to the species illustrated by Brady and by Cushman.

Distribution.-Single individuals found in only two core samples, $\mathrm{P}-49-61$ and $\mathrm{P}-52-61$.

\section{Genus EGGERELLA Cushman, 1933 \\ Eggerella bradyi (Cushman) \\ Plate 3, figure 4}

Verneuilina pygmaea (Egger). Brady, 1884, Challenger Repts., Zoology, v. 9, p. 385, pl. 47, figs. 4-7.

Verneuilina bradyi Cushman, 1911, U.S. Natl. Mus. Bull. 71, pt. 2, p. 54, 55, fig. 87 .

Eggerella bradyi (Cushman). Cushman, 1933, Cushman Lab. Foram. Research Contr., v. 9, p. 33.

Barker, 1960, Soc. Econ. Paleontologists and Mineralogists Spec. Pub. 9, p. 96, pl. 47, figs. 4-7.

Distribution. - Lat $37^{\circ}$ to $50^{\circ} \mathrm{N}$. Brady reported it as a common deep-water species, occurring in the North Pacific between depths of 3,400 and 5,700 meters.

\section{Eggerella scabra (Williamson) \\ Plate 3, figure 5}

Bulimina scabra Williamson, 1858, Recent British foraminifera, p. 65 , pl. 5, figs. $136,137$.

Verneuilina polystropha (Reuss). Brady, 1884, Challenger Repts., Zoology, v. 9, p. 386, pl. 47, figs. 15-17.

Verneuilina scabra (Williamson). Cushman, 1937, Cushman Lab. Foram. Research Spec. Pub. 8, p. 50, pl. 5, figs. 10, 11.

Barker, 1960, Soc. Econ. Paleontologists and Mineralogists Spec. Pub. 9, p. 96, pl. 47, figs. 15-17.

Distribution.-Rare, found north of lat $43^{\circ} \mathrm{N}$. Common in shallow water south of the Aleutian Islands. Deep-water forms are smaller and slightly less regular in shape.

\section{Family NUBECULARIIDAE Jones, 1875 Genus OPHTHALMIDIUM Kübler and Zwingli, 1870 \\ Ophthalmidium acutimargo (Brady)}

Spiroloculina acutimargo Brady, 1884, Challenger Repts. Zoology, v. 9, p. 154, pl. 10, fig. 13 (not figs. 14, 15)

Cushman, 1917, U.S. Natl. Mus. Bull. 71, pt. 6, p. 31, 32, pl. 1 , fig. 1.

Spirophthalmidium acutimargo (Brady). Cushman, 1927, Cushman Lab. Foram. Research Contr., v. 3, pt. 1, p. 37.

Barker, 1960, Soc. Econ. Paleontologists and Mineralogists Spec. Pub. 9, p. 20, pl. 10, fig. 13.

Distribution.-Rare in one core sample, P-52-61 (lat $44^{\circ} \mathrm{N}$, depth $5,160 \mathrm{~m}$ ).

\section{Ophthalmidium pusillum (Earland)}

\section{Plate 3, figure 7}

Spiroloculina tenuis (Czjzek). Brady, 1884, Challenger Repts., Zoology, v. 9, p. 152, pl. 10, figs. 9, 10 (not figs. $7,8,11$ ).

Spiroloculina pusillum Earland, 1934, Discovery Repts., v. 10 Foraminifera, pt. 3 , p. 47,48 , pl. 1, figs. 3,4 .

Spirophthalmidium pusillum (Earland). Barker, 1960, Soc. Econ. Paleontologists and Mineralogists Spec. Pub. 9, p. 20 , pl. 10, figs. 9,10 .

Distribution.-Lat $37^{\circ}$ to $41^{\circ} \mathrm{N}$ 
Family MILIOLIDAE Ehrenberg, 1839

Genus QUINQUELOCULINA Cushman, 1917

Quinqueloculina sp.

This species is very tiny and compressed; the sutures are only very slightly depressed. Only a single specimen was found.

Distribution.-Core sample P-38-61 (lat $46^{\circ} \mathrm{N}$., depth $5,610 \mathrm{~m}$ ).

\section{Genus PYRGO Defrance, 1824}

\section{Pyrgo sp.}

This specimen is possibly referrable to Pyrgo murrhyna (Schwager) (1866, Novara Exped., 18571859, Wien, Geol. Theil, Bd. 2, Abt. 2, p. 203, pl. 4, fig. 15), but the aperture is broken on the single specimen found.

Distribution.-Core sample $\mathrm{P}-16-61$ (lat $54^{\circ} \mathrm{N}$., depth $2,410 \mathrm{~m}$ ).

\section{Genus MILIOLINELLA Weisner, 1931}

Miliolinella subrotunda (Montagu)

Plate 3 , figure 8

Miliolina circularis (Bornemann). Brady, 1884, Challenger Repts., Zoology, v. 9, p. 169, pl. 4, fig. 3; pl. 5, figs. 13, 14.

Miliolinella subrotunda (Montagu) Weisner, 1931, Deutsche Sudpolar Exped., 1901-1903, v. 20 (Zoology, v. 12), p. 107.

Barker, 1960, Soc. Econ. Paleontologists and Mineralogists Spec. Pub. 9, p. 8, 10, pl. 4, fig. 3; pl. 5, figs. 13, 14.

Distribution.-Lat $30^{\circ}$ to $53^{\circ} \mathrm{N}$. Brady's reports are from shallow water. However, under Miliolina labiosa (d'Orbigny) (Brady, 1884, p. 170) he discusses deep-water specimens very similar to $M$. subrotunda, which may be the same species discussed here.

\section{Genus AMMOMASSILINA Cushman, 1933}

Ammomassilina alveolinaformis (Millett)

Spiroloculina asperula Karrer. Brady, 1884, Challenger Repts., Zoology, v. 9, p. 152, pl. 8, figs. 13, 14.

Massilina asperula (Karrer). Cushman, 1921, U.S. Natl. Mus. Bull. 100, v. 4, p. 447, 448.

Massilina alveolinaformis Millett. Cushman, 1928, U.S. Natl. Mus. Bull. 104, pt. 6, p. 39.

Ammomassilina alveolinaformis (Millett). Cushman, 1933, Cushman Lab. Foram. Research Contr., v. 9, pt. 2, p. 32.

Barker, 1960, Soc. Econ. Paleontologists and Mineralogists Spec. Pub. 9, p. 16, pl. 8, figs. 13, 14.

Distribution.-One specimen (corroled) found in core sample P-29-61 (lat $32^{\circ} \mathrm{N}$., depth $4,810 \mathrm{~m}$ ).
Famiy TURRILINIDAE Cushman, 1927

Genus BULIMINELLA Cushman, 1911

Buliminella basicostata Parr

Buliminella elegantissima d'Orbigny var. seminuda Terquem. Brady, 1884, Challenger Repts., Zoology, v. 9, p. 403, pl. 1, figs. 23, 24.

Buliminella basicostata Parr, 1950, Foraminifera, $B A N Z$ Antarctic Research Exped., 1929-1931, Repts., ser. B, v. 5, pt. 6, p. 336, pl. 12, figs. 11, 12 .

Barker, 1960, Soc. Econ. Paleontologists and Mineralogists Spec. Pub. 9, p. 104, pl. 50, figs. 23, 24.

Distribution.-Aleutian Terrace.

\section{Family BOLIVINITIDAE Cushman, 1923 Genus BOLIVINA d'Orbigny \\ Bolivina robusta Brady}

Bolivina robusta Brady, 1881, Micros. Sci. Quart. Jour., new ser., v. 21, p. 57.

Brady, 1884, Challenger Repts., Zoology, v. 9, p. 421, pl. 53, figs. 7-9.

Cushman, 1911, U.S. Natl. Mus. Bull. 71, pt. 2, p. 36, 37, figs. 59, 60.

Barker, 1960, Soc. Econ. Paleontologists and Mineralogists Spec. Pub. 9, p. 108, pl. 53, figs. 7-9.

Distribution.-One specimen (stained) from core sample P-40-61 (lat $50^{\circ}$ N., depth 5,500 m). Brady described it from generally less than 1,500 meters.

\section{Bolivina decussata Brady}

Bolivina decussata Brady, 1881, Micros. Sci. Quart. Jour., v. 21, no. 5, p. 58.

Brady, 1884, Challenger Repts., Zoology, v. 9, p. 406, p. 423, pl. 53, figs. 12, 13.

Barker, 1960, Soc. Econ. Paleontologists and Mineralogists Spec. Pub. 9, p. 110, pl. 53, figs. 12, 13.

Distribution.-Aleutian Terrace.

\section{Bolivina pseudoplicata Heron-Allen and Earland}

Bolivina plicata Brady, 1870, Ann. Mag. Nat. History, ser. 4 , v. 6 , p. 302 , pl. 12 , fig. 7 .

Bolivina plicata Halkyard, 1889, Manchester Micros. Soc. Trans. and Ann. Rept., v. 6, p. 61, pl. 1, fig. 13.

Bolivina pseudoplicata Heron-Allen and Earland, 1930, Royal Micros. Soc. London, ser. 3, v. 50, p. 81, pl. 3, figs. 36-49.

Distribution.-Lat $55^{\circ}$ to $60^{\circ} \mathrm{N}$, occurs only on Aleutian Terrace.

\section{Genus BULIMINA d'Orbigny, 1826 \\ Bulimina aculeata d'Orbigny \\ Plate 3, figure 9}

Bulimina aculeata d'Orbigny, 1826, Annales des Sci. Naturelles, v. 7, no. 7, p. 269.

Brady, 1884, Challenger Repts., Zoology, v. 9, p. 406, pl. 51, figs. 7-9.

Cushman, 1911, U.S. Natl. Mus. Bull. 71, pt. 2, p. 86, 87, fig. 139.

Barker, 1960, Soc. Econ. Paleontologists and Mineralogists Spec. Pub. 9, p. 104, pl. 51, figs. 7-9.

Distribution.-Rare in one core sample (P-16-61, lat $54^{\circ} \mathrm{N}$., depth $2,410 \mathrm{~m}$ ). 


\section{Genus GLOBOBULimina Cushman, 1927 \\ Globobulimina auriculata Bailey \\ Plate 3, figure 10}

Bulimina auriculata Bailey, 1851, Smithsonian Inst., Contr. Knowledge, v. 2, p. 12, pl., figs. 25-27.

Distribution.-Occurs rarely in core sample P-1661 (lat $54^{\circ} \mathrm{N}$, depth $2,410 \mathrm{~m}$ ). Two of the three specimens are stained.

\section{Globobulimina pacifica Cushman}

Bulimina pyrula d'Orbigny. Brady, 1884, Challenger Repts., Zoology, v. 9, p. 339, pl. 50, figs. 7-10.

Globobulimina pacifica Cushman, 1927, Cushman Lab. Foram. Research Contr., v. 3, p. 67, pl. 14, fig. 12.

Globobulimina pacifica Cushman?. Barker, 1960, Soc. Econ. Paleontologists and Mineralogists Spec. Pub. 9, p. 102, pl. 50, figs. 7-10.

Distribution.-One stained specimen in core sample P-16-61 (lat 54 ${ }^{\circ}$ N., depth 2,410 m) .

\section{Family UVIGERINIDAE Haeckel, 1894 \\ Genus UVIGERINA d'Orbigny, 1826}

Uvigerina peregrina Cushman

Plate 3, figure 11

Uvigerina pygmaea d'Orbigny. Brady, 1884, Challenger Repts., Zoology, v. 9, p. 575, pl. 74, figs. 11, 12.

Uvigerina peregrina Cushman, 1923, U.S. Natl. Mus. Bull. 104 , pt. 4, p. 166, pl. 42, figs. 7-11.

Euuvigerina peregrina (Cushman). Barker, 1960, Soc. Econ. Paleontologists and Mineralogists Spec. Pub. 9, p. 154, pl. 74, figs. 11, 12 .

Distribution.-Several specimens found in core sample P-16-61 (lat 54 ${ }^{\circ}$ N., depth 2,410 m).

\section{Uvigerina cushmani Todd}

Uvigerina cushmani Todd, 1948, in Cushman and McCulloch, 1948, Allan Hancock Pacific Exped. Repts., v. 6, no. 5 , p. 257 , pl. 33 , fig. 1 .

Distribution.-Aleutian Terrace.

\section{Genus ANGULOGERINA Cushman, 1927}

\section{Angulogerina fluens Todd}

Angulogerina angulosa (Williamson). Cushman, 1948 [not Uvigerina angulosa Williamson], Cushman Lab. Foram. Research Spec. Pub. 23, p. 66, pl. 7, fig. 8 .

Brady, 1884, Challenger Repts., Zoology, v. 9, p. 576, pl. 74, figs. 15, 16.

Barker, 1960, Soc. Econ. Paleontologists and Mineralogists Spec. Pub. 9, p. 154, pl. 75, figs. 15, 16.

Angulogerina fluens Todd, 1947, in Cushman and Todd, 1947, Cushman Lab. Foram. Research Contr., v. 23, pt. 3, p. 67, pl. 16 , figs. 6,7 .

Distribution.-Aleutian Terrace.

Family DISCORBIDAE Ehrenberg, 1838

Genus EPISTOMINELLA Husezima and Maruhasi, 1944

Epistominella exigua (Brady)

Plate 3, figure 12

Pulvinulina exigua Brady, 1884, Challenger Repts., Zoology, v. 9 , p. 696 , pl. 103 , figs. 13,14 .
Epistominella exigua (Brady). Barker, 1960, Soc. Econ. Paleontologists and Mineralogists Spec. Pub. 9, p. 212, pl. 103, figs. 13, 14.

Distribution.-Lat $44^{\circ}$ to $55^{\circ} \mathrm{N}$. This rare form is generally stained. According to Brady, it is generally found deeper than 1,800 meters.

Epistominella umbonifera (Cushman)

Plate 3, figure 13

Pulvinulinella umbonifera Cushman, 1933, Cushman Lab. Foram. Research Contr., v. 9, pt. 4, p. 90, pl. 9, fig. 9. not Epistominella? umbonifera (Cushman). Phleger, Parker, and Pierson, 1953, Repts. Swedish Deep-sea Exped., v. 7 , no. 1 , p. 43,44 , pl. 9 , figs. 33,34 .

Distribution.-Core sample P-49-61 (lat 50 $\mathrm{N}$., depth $5,000 \mathrm{~m}$ ). Two stained specimens, identical with Cushman's figures, occur at this station. Phleger, Parker, and Pierson's specimens have more numerous chambers. Cushman described the form from the South Pacific at a depth of 2,243 meters.

Family ELPHIDIIDAE Galloway, 1933

Genus ELPHIDIUM de Montfort, 1808

Elphidium incertum (Williamson)

Polystomella umbilicatula var. incerta Wiliamson, 1858, Recent British Foraminifera, p. 44, pl. 3, fig. 82a.

Elphidium incertum (Williamson). Loeblich and Tappan, 1953, Smithsonian Inst. Misc. Colln., v. 121, no. 7, p. 100-102.

Distribution.-This species occurs only in core sample P-16-61 (lat $54^{\circ} \mathrm{N}$., depth $2,410 \mathrm{~m}$ ) north of the Aleutian Trench. It appears identical with Williamson's figures. (See discussion in Loeblich and Tappan, 1953.)

\section{Elphidium magellanicum Heron-Allen and Earland}

Elphidium (Polystomella) magellanicum Heron-Allen and Earland, 1932, Discovery Repts., v. 4, p. 440, pl. 16, figs. 26-28.

Distribution.-Aleutian Terrace.

\section{Genus ELPHIDIELLA Cushman, 1936 \\ Elphidiella groenlandica (Cushman) \\ Plate 4, figure 1}

Elphidium groenlandicum Cushman, 1933, Smithsonian Inst. Misc. Colln., v. 89, no. 9, p. 4, pl. 1, fig. 10.

Elphidiella groenlandica (Cushman). Loeblich and Tappan, 1953, Smithsonian Inst. Misc. Colln., v. 121, no. 7, p. 106, 107, pl. 19, figs. 13, 14 .

Distribution.-This species is abundant in core sample P-16-61 (lat $54^{\circ} \mathrm{N}$., depth 2,410 m). Several specimens are stained.

\section{Family GLOBOROTALIIDAE Cushman, 1927 Geuus GLOBOROTALIA Cushman, 1927 \\ Globorotalia inflata (d'Orbigny)}

Globigerina inflata d'Orbigny, 1839, in Barker-Webb and 
Berthelot, Histoire Naturelle des l'îles Canaries, v. 2, pt. 2, Foraminifères, p. 134, pl. 2, figs. 7-9.

Brady, 1884, Challenger Repts., Zoology, v. 9, p. 601, pl. 79, figs. 8-10.

Barker, 1960, Soc. Econ. Paleontologists and Mineralogists Spec. Pub. 9, p. 164, pl. 79, figs. 8-10.

Globorotalia inflata (d'Orbigny). Parker, 1962, Micropaleontology, v. 8, no. 2, p. 236, pl. 5, figs. 6-9.

Distribution.-One specimen from core sample $\mathrm{P}$ 26-61 (lat $26^{\circ}$ N., depth 5,210 m).

\section{Family GLOBIGERINIDAE Carpenter, Parker, and Jones, 1862 Genus GLOBIGERINA d'Orbigny, 1826 \\ Globigerina bulloides d'Orbigny}

Globigerina bulloides d'Orbigny, 1826, Annals des Sci. Naturelles, ser. 1, v. 7, no. 1 .

Brady, 1884, Challenger Repts., Zoology, v. 9, p. 593, pl. 77; pl. 79, figs. 3-7.

Barker, 1960, Soc. Econ. Paleontologists and Mineralogists Spec. Pub. 9, p. 164, pl. 77; pl. 79, figs. 3-7.

Parker, 1962, Micropaleontology, v. 8, no. 2, p. 221, pl. 1, figs. 1-8.

Distribution.-Lat $23^{\circ}$ to $55^{\circ}$ N. Rare, often corroded.

Genus CANDEINA d'Orbigny in de La Sagra, 1839

\section{Candeina nitida d'Orbigny}

Candeina nitida d'Orbigny, 1839, in de la Sagra, Histoire physique, politique et naturelle de l'île de Cuba, Foraminifères, p. 108, pl. 2, figs. 27, 28.

Brady, 1884, Challenger Repts., Zoology, v. 9, p. 622, pl. 82, figs. 13-20.

Barker, 1960, Soc. Econ. Paleontologists and Mineralogists Spec. Pub. 9, p. 170, pl. 82, figs. 13-20.

Parker, 1962, Micropaleontology, v. 8, no. 2, p. 253, pl. 8, figs. 27-30.

Distribution.-One specimen found in core sample P-56-61 (lat $39^{\circ} \mathrm{N}$., depth 5,400 m) .

\section{Family CIBICIDIDAE Cushman, 1927 \\ Genus CIBICIDES de Montfort, 1808 \\ Cibicides bradyi (Trauth) \\ Plate 4, figure 2}

Truncatulina dutemplei (d'Obrigny). Brady, 1884, Challenger Repts., Zoology, v. 9, p. 665, pl. 95, fig. 5.

Truncatulina bradyi Trauth, 1918, K. Acad. Wiss. Wien, Math.-Naturw. Cl., Denkschr., Wien, v. 95, p. 235.

Cibicides bradyi (Trauth). Thalmann, 1942, Am. Midland Naturalist, v. 28, no. 2, p. 464.

Barker, 1960, Soc. Econ. Paleontologists and Mineralogists Spec. Pub. 9, p. 196, pl. 95, fig. 5.

Distribution.-Lat $32^{\circ}$ to $52^{\circ}$ N. Rare but widely distributed. Except for a few specimens in core sample P-19-61 (lat $32^{\circ}$ N.), all are stained.

Cibicides lobatulus (Walker and Jacob)

Cibicides lobatulus (Walker and Jacob). Barker, 1960, Soc. Econ. Paleontologists and Mineralogists Spec. Pub. 9, p. 192, pl. 93, figs., $1,4,5$.

Distribution.-Aleutian Terrace.

\section{Family CASSIDULINIDAE d'Orbigny, 1839 \\ Genus CASSIDULINA d'Orbigny, 1826 \\ Cassidulina subglobosa Brady \\ Plate 4, figure 3}

Cassidulina subglobosa Brady, 1881, Micros. Sci. Quart. Jour., new ser., v. 21, p. 60.

Brady, 1884, Challenger Repts., Zoology, v. 9, p. 430, pl. 54, fig. 17 .

Barker, 1960, Soc. Econ. Paleontologists and Mineralogists Spec. Pub. 9, p. 111, pl. 54, fig. 17.

This rare form is smaller than that described by Brady. The specimens may represent immature forms of Brady's species.

Distribution.-Lat $55^{\circ}$ N., depth 4,430 meters. One specimen, stained.

\section{Cassidulina crassa d'Orbigny}

Cassidulina crassa d'Orbigny, 1839, Voyage dans l'Amerique Meridionale, v. 5, pt. 5, p. 56, pl. 7, figs. 18-20.

Brady, 1884, Challenger Repts., Zoology, v. 9, p. 429-430, pl. 54, figs. 4, 5 .

Barker, 1960, Soc. Econ. Paleontologists and Mineralogists Spec. Pub. 9, p. 110, pl. 53, figs. 4, 5.

Distribution.-Aleutian Terrace.

\section{Cassidulina teretis Tappan}

Cassidulina laevigata d'Orbigny. Brady, 1884, Challenger Repts., Zoology, v. 9, p. 428, pl. 54, figs. 1-3.

Cassidulina teretis Tappan, 1951, Cushman Found. Foram. Research Contr., v. 2, pt. 1, p. 7, pl. 1, figs. 3a-c.

Loeblich and Tappan, 1953, Smithsonian Misc. Colln., v. 121 , no. 7 , p. 121, pl. 24, figs. 3,4 .

Barker, 1960, Soc. Econ. Paleontologists and Mineralogists Spec. Pub. 9, p. 110, pl. 53, fig. 1.

Distribution.-Aleutian Terrace.

\section{Cassidulina tortuosa Cushman and Hughes}

Cassidulina tortuosa Cushman and Hughes, 1925, Cushman Lab. Foram. Research Contr., v. 1, p. 14, pl. 2, fig. 4. Distribution.-Aleutian Terrace.

\section{Genus EHRENBERGINA Reuss, 1850 \\ Ehrenbergina hystrix Brady \\ Plate 4, figure 5}

Ehrenbergina hystrix Brady, 1881, Micros. Sci. Quart. Jour., new ser., v. 21, p. 60.

Brady, 1884, Challenger Repts., Zoology, v. 9, p. 434, pl. 55, figs. 8-11.

Barker, 1960, Soc. Econ. Paleontologists and Mineralogists Spec. Pub. 9, p. 112, pl. 55, figs. 8-11.

Distribution.-One specimen in core sample $\mathrm{P}$ 49-61 (lat $50^{\circ} \mathrm{N}$., depth 5,000 m). Brady described it as occurring rarely only in the deep water of the South Pacific. 
Family INVOLUTINIDAE Bütschli, 1880 Genus INVOLUTINA Terquem, 1862

Involutina tenuis (Brady)

Plate 4, figure 6

Ammodiscus tenuis Brady, 1881, Micros. Sci. Quart. Jour., new ser., v. 21, p. 51.

Brady, 1884, Challenger Repts., Zoology, v. 9, p. 332, pl. 38 , figs. 4-6.

Involutina tenuis (Brady). Barker, 1960, Soc. Econ. Paleontologists and Mineralogists Spec. Pub. 9, p. 78, pl. 38 , figs. 5, 6.

Distribution.-Lat $39^{\circ}$ to $53^{\circ} \mathrm{N}$. The test is thin walled and variable in amount of contortion.

\section{Family NONIONIDAE Schultze, 1854 Genus NONION de Montfort, 1808}

Nonion labradoricum (Dawson)

Plate 4, figure 4

Nonionina labradorica Dawson, 1860, Canadian Naturalist, v. 5, p. 191, fig. 4.

Nonion labradoricum (Dawson). Loeblich and Tappan, 1953, Smithsonian Inst. Misc. Colln., v. 121 , no. 7, p. 86, 87, pl. 17, figs. 1, 2.

Distribution.-Only present in core sample P-1661 (lat $54^{\circ} \mathrm{N}$., depth $2,410 \mathrm{~m}$ ), where all specimens are stained. It is also present in shallower water samples south of the Aleutian Islands.

\section{Nonion scaphum (Fichtel and Moll)}

Nonionina scaphum (Fichtel and Moll). Brady, 1884, Challenger Repts., Zoology, v. 9, p. 730, pl. 109, figs. 14, 15. Nonion scaphum (Fichtel and Moll). Barker, 1960, Soc. Econ. Paleontologists and Mineralogists Spec. Pub. 9, p. 224, pl. 109, figs. 14, 15 .

Distribution.-Aleutian Terrace.

\section{Genus NONIONELLA Cushman, 1926 \\ Nonionella turgida (Williamson) \\ Plate 4, figure 8}

Rotalina turgida Williamson, 1858, Recent British Foraminifera, p. 50, pl. 4, figs. 95-97.

Nonionina turgida (Williamson). Brady, 1884, Challenger Repts., Zoology, v. 9, p. 731, pl. 109, figs. 17-19.

Nonionella turgida (Williamson). Barker, 1960, Soc. Econ. Paleontologists and Mineralogists Spec. Pub. 9, p. 224, pl. 109, figs. 17-19.

Distribution.-Found only in core sample P-1661 (lat $54^{\circ} \mathrm{N}$., depth $2,410 \mathrm{~m}$ ). All specimens are stained.

\section{Nonionella bradyi (Chapman)}

Nonion scapha (Fichtel and Moll). Brady, 1884, Challenger Repts., Zoology, v. 9, p. 224, pl. 109, fig. 16.

Nonionella bradyi (Chapman). Barker, 1960, Soc. Econ. Paleontologists and Mineralogists Spec. Pub. 9, p. 224, pl. 109, fig. 16.

Distribution.-Aleutian Terrace.

Nonionella auricula Heron-Allen and Earland

Nonionella auricula Heron-Allen and Earland, 1930, Royal Micros. Soc. London Jour., ser. 3, v. 50, p. 192, pl. 5, figs. 68-70.

Loeblich and Tappan, 1953, Smithsonian Misc. Colln., v. 121, no. 7 , p. 92,93 , pl. 16, figs. 6-10.

Distribution.-Aleutian Terrace.

Genus PULLENIA Parker and Jones, in Carpenter, Parker, and Jones, 1862

Pullenia subcarinata (d'Orbigny)

Plate 4, figure 9

Nonionina subcarinata d'Orbigny, 1839, Voyage dans l'Amerique Meridionale, v. 5, pt. 5, Foraminifères, p. 28, pl. 5, figs. 23, 24.

Pullenia quinqueloba Reuss. Brady, 1884, Challenger Repts., Zoology, v. 9, p. 617, pl. 84, figs. 14, 15 .

Pullenia subcarinata (d'Orbigny). Heron-Allen and Earland, 1932, Discovery Repts., v. 4, Foraminifera, pt. 1, p. 403, pl. 13, figs. 14-18.

Barker, 1960, Soc. Econ. Paleontologists and Mineralogists Spec. Pub. 9, p. 174, pl. 84, figs. 14, 15.

Distribution.-Lat $37^{\circ}$ to $53^{\circ} \mathrm{N}$. Widely distributed in deep water, generally represented by only a few specimens, nearly always stained.

\section{Family ALABAMINIDAE Hofker, 1951 Genus GYROIDINA d'Orbigny, 1826 \\ Gyroidina lamarckiana (d'Orbigny) \\ Plate 4, figure 7}

Rotalina lamarckiana d'Orbigny, 1839, in Barker-Webb and Berthelot, Histoire Naturelle des l'îles Canaries, v. 2, pt. 2, Foraminifères, p. 131, pl. 2, figs. 13-15.

Gyroidina lamarckiana (d'Orbigny). Phleger, Parker, and Pierson, 1953, Repts. Swedish Deep-sea Exped., v. 7, pt. 1 , p. 40 , pl. 8 , figs. 33,34 .

Distribution. - One stained specimen found in core sample P-49-61 (lat 50 ${ }^{\circ}$ N., depth 5,000 m). Phleger, Parker, and Pierson list this species from six stations in the North Atlantic, all deeper than 4,000 meters.

\section{Family ANOMALINIDAE Cushman, 1927 Genus ANOMALINA d'Orbigny, 1826 \\ Anomalina globulosa Chapman and Parr Plate 4, figure 10}

Anomalina grosserugosa (Gumbel). Brady, 1884, Challenger Repts., Zoology, v. 9, p. 673, pl. 94, figs. 4, 5.

Anomalina globosa Chapman and Parr, 1937, Australasian Antarctic Exped., 1911-1914, Sci. Repts., Ser. C, v. 1, pt. 2, p. 117, pl. 9, fig. 27.

Anomalina globulosa Chapman and Parr. Barker, 1960, Soc. Econ. Paleontologists and Mineralogists Spec. Pub. 9, p. 194 , pl. 94 , figs. 4,5 .

A few stained specimens that are apparently referrable to this species occur in core sample P-1261 , although the sutures are less distinct and the outline is smooth.

Distribution.-Lat $53^{\circ}$ N., depth 6,560 meters. 
Genus CIBICIDOIDES Thalmann, 1939

Cibicidoides cf. C. mundulus (Brady, Parker, and Jones)

Plate 4, figure 13

Truncatulina sp. Brady, 1884, Challenger Repts., Zoology, v. 9 , pl. 95, fig. 6 .

Cibicides mundulus (Brady, Parker, and Jones). Chapman and Parr, 1937, Australasian Antarctic Exped., Sci. Repts., Ser. C, v. 1, pt. 2, p. 120.

Cibicidoides mundulus (Brady, Parker, and Jones). Barker, 1960, Soc. Econ. Paleontologists and Mineralogists Spec. Pub. 9, p. 196, pl. 95, fig. 6 .

This form appears identical to that illustrated by Brady, except for its small size. The single specimen found was stained.

Distribution.-Lat $50^{\circ}$ N., depth 5,000 meters.

\section{Genus MELONIS de Montfort, 1808}

Melonis affine (Reuss)

Plate 4, figure 12

Nonionina affinis Reuss, 1851, Deutsche Geol. Gesell. Zeitschr., v. 3, p. 72, pl. 5, fig. 32 .

Nonionina umbilacatula (Montagu). Brady, 1884, Challenger Repts., Zoology, v. 9, p. 726, pl. 109, figs. 8, 9.

Nonion affine (Reuss). Boltovskoy, 1958, Micropaleontology, v. 4 , p. $193-200$.

Gavelinonion barleeanum (Williamson). Barker, 1960, Soc. Econ. Paleontologists and Mineralogists Spec. Pub. 9, p. 224, pl. 109, figs. 8, 9.

Distribution.-One small stained specimen found (core sample $\mathrm{P}-49-61$, lat $50^{\circ} \mathrm{N}$., depth 5,000 m).

\section{Melonis pompilioides (Fichtel and Moll) \\ Plate 4, figure 11}

Nautilus pompilioides Fichtel and Moll, 1798, Testacea Microscopica, p. 31, pl. 2, figs. a-c.

Nonionina pompilioides (Fichtel and Moll). Brady, 1884, Challenger Repts., Zoology, v. 9, p. 727, pl. 109, figs. 10, 11.

Nonion? pompilioides (Fichtel and Moll). Barker, 1960, Soc. Econ. Paleontologists and Mineralogists Spec. Pub. 9, p. 224, pl. 109, figs. 10, 11.

Distribution.-One specimen found in core sample P-29-61 (lat $32^{\circ} \mathrm{N}$., depth $4,810 \mathrm{~m}$ ). It is very small but is referred to $N$. pompilioides because of its thickness and coarsely perforate wall.

\section{Family CERATOBULIMINIDAE Cushman, 1927 Genus HOEGLUNDINA Brotzen, 1948 \\ Hoeglundina elegans (d'Orbigny)}

Rotalia elegans d'Orbigny, 1826, Annals Sci. Naturelles, ser. 1 , v. 7 , no. 6 , p. 272.

Pulvinulina elegans (d'Orbigny). Brady, 1884, Challenger Repts., Zoology, v. 9, p. 699, pl. 105, figs. 3-6.

Höglundina elegans (d'Orbigny). Barker, 1960, Soc. Econ. Paleontologists and Mineralogists Spec. Pub. 9, p. 216, pl. 106, figs. 3-6.

Distribution.-Lat $32^{\circ}$ to $54^{\circ} \mathrm{N}$. All specimens are small and, when not stained, appear corroded.

\section{REFERENCES CITED}

Barker, R. W., 1960, Taxonomic notes on the species figured by H. B. Brady in his report on the Foraminifera dredged by HMS Challenger during the years 1873 to 1876: Soc. Econ. Paleontologists and Mineralogists Spec. Pub. 9, 240 p.

Brady, H. B., 1884, Report on the Foraminifera dredged by HMS Challenger during years 1873-1876: Challenger Repts., Zoology, v. 9, p. i-xxi, 1-814.

Bukry, David, Douglas, R. G., Kling, S. A., and Krashininnikov, Valeri, 1971, Planktonic microfossil biostratigraphy of the northwestern Pacific Ocean: Deep Sea Drilling Proj. Initial Repts., v. 6, p. 1253-1300.

Cushman, J. A., and McCulloch, Irene, 1939, A report on some arenaceous Foraminifera: Univ. Southern California, Allan Hancock Pacific Exped. Repts., v. 6, no. 1, $113 \mathrm{p}$.

Krashininnikov, V. A., 1971, Cenozoic Foraminifera: Deep Sea Drilling Proj. Initial Repts., v. 6, p. 1055-1068.

Lisitzen, A. P., 1971, Distribution of carbonate microfossils in suspension and in bottom sediments; The micropaleontology of oceans: Cambridge Univ. Press, p. 197-218.

Olson, R. K., and Goll, R., 1970, Biostratigraphy: Deep Sea Drilling Proj. Initial Repts., v. 5, p. 557-567.

Revelle, Roger, 1944, Marine bottom samples collected in the Pacific Ocean by the Carnegie on its seventh cruise: Carnegie Inst. Washington Pub. 556, 180 p.

Riedel, W. R., and Funnell, B. M., 1965, Tertiary sediment cores and microfossils from the Pacific Ocean floor: Geol. Soc. London Quart. Jour., v. 120, p. 305-368. 



\section{INDEX}

[Italic page numbers indicate major references]

\section{$\mathbf{A}$}

Page

abyssorum, Rhabdammina _-_...- 6, 12 Acknowledgments

aculeata, Bulimina acuta, Jaculella _-_.-- 6, 8, 9, 10,11, 12; pl. 1 acutimargo, Ophthalmidium _..- 6,9,11, 18 Spiroloculivia _-_._-_-_._-_- 18 Spirophthalmidium - 18

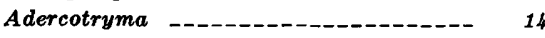
glomerata glomeratum _...- 8,9,10,11, 12,14; pl. 1 advena, Eggerella _._._._. 10 affine, Melonis _. Nonion -..-_- 23

affinis, Bulimina _... 11 Nonionina --.-_-_-_-_-_-_- 23 agglutinans, Ammobaculites _.- 6,9,16; pl. 2 biformis, Textularia filaformis, Ammobaculites_ 6, 8, 10, 16; pl. 2 Haplophragmium -_. 16 Spirolina

Alabaminidae

Aleutian Islands

Aleutian Terrace

Aleutian Trench _........ 1, 6, 7 alveolinaformis, Ammomassilina Massilina

Alveolophragmium nitidum

$8,9,10,11,12,15 ; \mathrm{pl} .1$

ringens _._........... $6,8,9,10,15$

scitulum _-_._._- 6, 8, 9, 12, 15; pl. 1

subglobosum - 6,7,8,9,10,11, 12,15; pl. 1 weisneri _..._. $6,8,9,10,15 ; \mathrm{pl} .2$ sp -...- 6,$15 ; \mathrm{pl} .2$ americanus, Ammobaculites - 6,8,9,10,12,16 Ammobaculites _. agglutinans _....... 6, 9, 16; pl. 2 filaformis _..... $6,8,10,16 ; \mathrm{pl} .2$ americanus _..._- 6, 8, 9, 10,11,12,16 filaformis

Ammochilostoma galeata _.......-. 18

Ammodiscidae --_-_-_-_-_-_-_-_- 19

Ammodiscus gordialis tenuis _..._. 22

Ammoglobigerina bulloides _._-_-_ $\quad 17$ globigeriniformis _-_._._- 17

Ammomarginulina _._._._._._. 16 foliacea _....... $6,8,10,16 ; \mathrm{pl} .2$ foliaceus --

Ammomassilina _. alveolinaformis _...... 6, 19

Ammoscalaria tenuimargo _-_-_-_-_-- 10,11 amygdaliformis, Loxostomum -..-.- 7 anceps, Globotextularia _. Haplophragmium _._. 18

Angulogerina angulosa _... fluens -angulosa, Angulogerina _....... 6, 7, 20 Uvigerina _.... 20

Anomalina globosa -globulosa -..-_grosserugosa
[Italic page numbers indicate major references]
Page

Anomalinidae

arctica, Labrospira

Aschemonella sp

19

Spiroloculina _- 19

Astrorhiza - 8

sp _.

Astrorhizidae _-_._-_._- 12

Ataxophragmiidae

atlantica, Pseudogaudryina _._._._ 6, 7

auricula, Nonionella _._. auriculata, Bulimina Globobulimina

$\mathbf{B}$

Baculogypsina _._. 8,9 sp _._._. 10 barleeanum, Gavelinonion bartletti, Elphidium _-_- 7, 8 basicostata, Buliminella _..._... 6, 7, 19 Bathysiphon discreta

Bibliography _._-_._- 23

biformis, Spiroplecta _- 16 Spiroplectammina --- 6, 8, 9, 10,16; pl. 2 Textularia agglutinans _._._..._ 16

Bigenerina - 16 minutissima _.

Bolivina - _. decussata _. plicata _.._-_._._- 19 pseudoplicata robusta _...

Bolivinitidae _... 19

bradyi, Cibicides _... 6, 8, 9, 11, 21; pl. 4 Cyclammina _-_._. 14 Eggerella _..- 6, 8, 9, 10,11, 12, 18; pl. 3 Eponides _._._._._. 9 Nonionella Placopsilina _. Truncatulina _... 21 Verneuilina

Buccella frigida inusitata

Bulimina - 19 aculeata _. affinis auriculata _. mexicana _._. 8 pyrula _... 20 scabra

Buliminella basicostata _..._. $6,7,19$ elegantissima _..- 7 seminuda --1.- 19

bulloides, Ammoglobigerina Globigerina _........ 6, 7, 8, 9, 11, 21

\section{o}

canariensis, Haplophragmoides _-_-_- 7, 8 cancellata, Cyclammina - 6,

Candeina $9,10,11,12,14 ;$ pl. 1 nitida

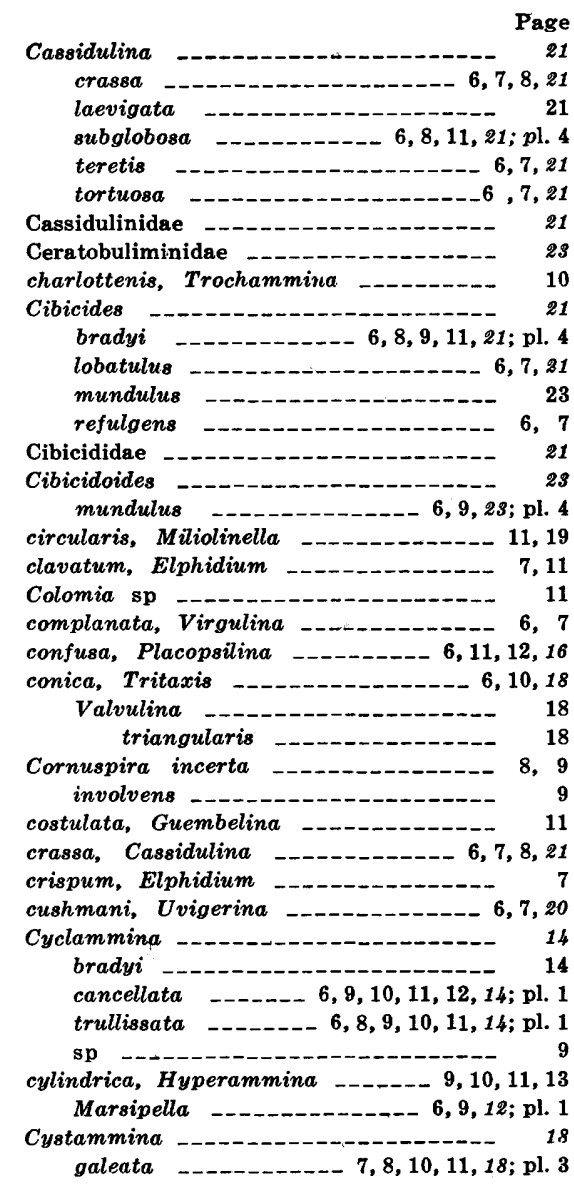

D

decussata, Bolivina $6,7,19$ dentalinaformis, Reophax

Diatoms

difflugiformis, Proteonina - 13 Reophax _-_ $6,8,9,10,11,12,13 ;$ pl. 1 Discorbidae _........ 20 Discorbis rosea _..._. 9 discreta, Bathysiphon _._. 6,$12 ; \mathrm{pl} 1$ Psammosiphonella _-_._-_-_- 12 Rhabdammina _._._._._. 12 distans, Reophax - 6, 10, 13; pl. 1 Dorothia _...... 18 exilis _... dutemplei, Truncatulina

\section{$\mathbf{E}$}

Eggerella _advena _._._. bradyi _-_-_ 6,8,9,10,11,12.18; pl. 3 fusca --_----_- 10 propinqua _... Pl. 3 scabra _. 


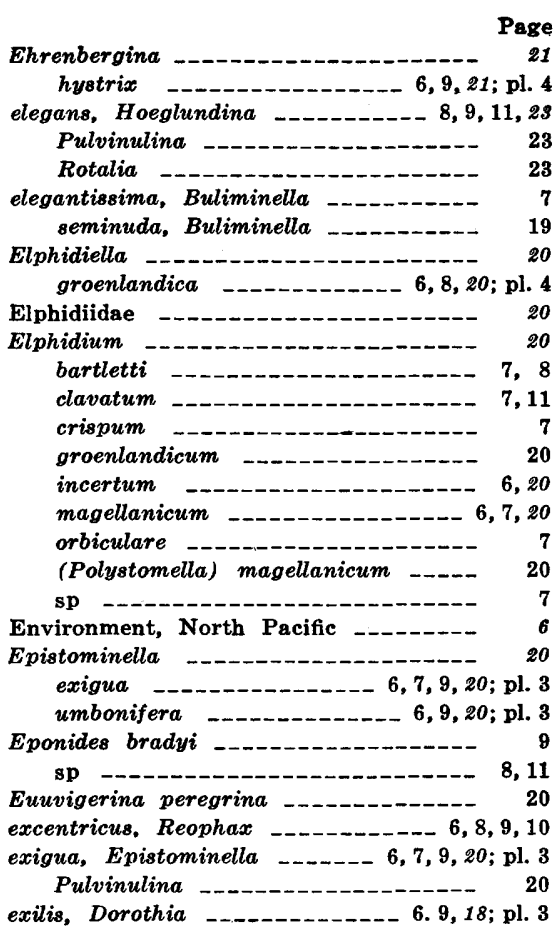

\section{F}

filaformis, Ammobaculites _...- 6, 8,9,10,11 Ammobaculites agglutinans - 6 , Gaudryina $8,10,16 ;$ pl. 2 Fissurina sp fluens, Angulogerina -_... 20 foliacea, Ammomarginulina _- 6, 8, 10, 16; pl. 2 foliaceum, Haplophragmium _-_._. 16 foliaceus, Ammomarginulina -..--_ 16 fontinense, Haplophragmium _....- 16 Foraminifera friabilis, Hyperammina frigida, Buccella fusca, Eggerella

Psammosphaera

\section{G}

galeata, Ammochilostoma -..-_-_- 18 Cystammina _-_- $7,8,10,11,17,18$; pl. 3 Trochammina - 18

Gaudryina filaformis _._._._._- 18

Gavelinonion barleeanum _-_-_---- 23

Globigerina _..._. 21

bulloides _. inflata _... 11 pachyderma -

Globigerinidae -

globigeriniforme, Haplophragmium -- 17 globigeriniformis, Ammoglobigerina --- 17 Lituola nautiloidea Trochammina --.- 7, 8, 9, 10,11, 17; pl. 3 Globigerinita voluta Globobulimina _auriculata _._. pacifica Globorotalia -inflata

Globorotaliidae -

globosa, Anomalina - 22

Globotextularia -_anceps sp
Globotruncana marginata $\quad$ Page

sp - globulifera, Hormosina $-6,9,10,11,12,15,14$ globulosa, Anomalina _....... 6, 22; pl. 4 glomerata, Adercotryma _._._._-_ 6,14 Lituola -_-_-_-_-_-_-_-_- 14 glomeratum, Adercotryma - 8 , Glomospira $9,10,11,12,14$; pl. 1 gordialis gordialis, Ammodiscus _..... 13

Glomospira _- $6,8,9,10,11,12,18 ; \mathrm{pl} .1$

Gordiammina _.. 13

Trochammina squamata _-_._- 13

Gordiammina gordialis _....... 13

grisea, Trochammina ---- 7, 8, 9, 10, 17; pl. 2 groenlandica, Elphidiella _-.- 6, 8,20; pl. 4 groenlandicum, Elphidium _..._...- 20 grosserugosa, Anomalina --.---_--- 22 Guembelina costulata globulosa sp _...... 11

Gyroidina --.-- 22 lamarckiana _-_._-_ 6,9, 22; pl. 4

\section{$\mathbf{H}$}

Haplophragmium agglutinans anceps

foliaceum

fontinense

globigeriniforme

latidorsatum

nanum

nitidum

-

turbinatum helicoideum

Haplophragmoides canariensis _-_.-- 7, 8 nitida -

nitidum _._.

nitidus

ringens

subglobosin

helicoideum, Haplophragmium turbinatum --_--_-_-_ 17

Hoeglundina _............... 28 elegans Hormosina -..- 13 globulifera _..... 6, 9,10,11,12,1s, 14 normani

normani
Hormosinidae

- 13

作 11,12 cylindrica _._._._._._. $9,10,11,13$ friabilis spp -

hystrix, Ehrenbergina _-_._.-- 6, 9, 21; pl. 4

I

incerta, Cornuspira

Polystomella umbilicatula incertum, Elphidium _. 6,20 inflata, Globigerina _... 11

Globorotalia _............. 6,20 Trochammina inusitata, Buccella _-_._- 7 Involutina _tenuis Involutinidae

involvens, Cornuspira

\section{J $\mathbf{K}$}

Jaculella acuta kellettae, Trochammina
Kodiak Harbor

Page

Kodiak Island

7

\section{$\mathbf{L}$}

Labiosa, Miliolina labradorica, Nonionina - 22 labradoricum, Nonion _..- 6,7,8,11, 22; pl. 4 Labrospira aretica _..._- 15 subglobosa weisneri

laevigata, Cassidulina lamarckiana, Gyroidina Rotalina _-_._-________-_-- 22 latidorsatum, Haplophragmium _.... 15 linearis, Oculosiphon _- 7, 8 lingua, Pseudopolymorphina Lituola glomerata _-_-_-_-_._- 14 nautiloidea globigeriniformis _..- 17 Lituolidae lobatulus, Cibicides _-_-_-_-_ 6, 7, 21 Loxostomum amygdaliformis _._._-_ 7

\section{$\mathbf{M}$}

magellanicum, Elphidium Elphidium (Polystomella) -_-_-- 20 malovensis, Trochammina ---- 7,15, 17; pl. 2 marginata, Globotruncana _-_._- 11 Marsipella --_-_-_-_- 12 cylindrica

Massilina alveolinaformis _._._. 19 asperula _...... 19

Melonis affine pompilioides _..._._._- 6, 11, 2s; pl. 4 membranaceum, Nodellum _._-__- 7, 8; pl. 1 mexicana, Bulimina Miliolidae Miliolina labiosa _-_-_-_-_-_ 19 Miliolinella circularis _. subrotunda _.... 6, 8,9,10,11, 19 ; pl. 3 minutissima, Bigenerina $---6,9,10,16 ; \mathrm{pl} .2$ mundulus, Cibicides _- 23 Cibicidoides _._._._-_-_ 6, 9, 23; pl. 4

$\mathbf{N}$

nana, Trochammina _-___- 7,9,17; pl. 2 nanum, Haplophragmium nautiloidea globigeriniformis, Lituola Nautilus pompilioides _-_-_-_-_-- 23 nitida, Candeina _. Haplophragmoides -_._._._. 7, 8 Trochammina - $7,9,10,11,12,17$; pl. 2 nitidum, Alveolophragmium _... 6 , $8,9,10,11,12,15 ;$ pl. 1 Haplophragmium ------_---- 15 Haplophragmoides -_._-_...- 15 nitidus, Haplophragmoides _-..-- 15 Nodellum membranaceum _-_-_-- 7, 8; pl. 1 nodulosa, Reophax _._. 14 nodulosus, Reophax --- 6, 8, 9, 10, 12, 14; pl. 1 Nonion _affine labradoricum _..... 6, 7, 8,11,22; pl. 4 pompilioides _-

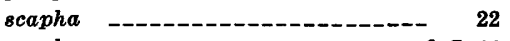
8caphum _. $6,7,22$ sp _-_-

Nonionella auricula bradyi _._._. turgida _...... Nonionidae 


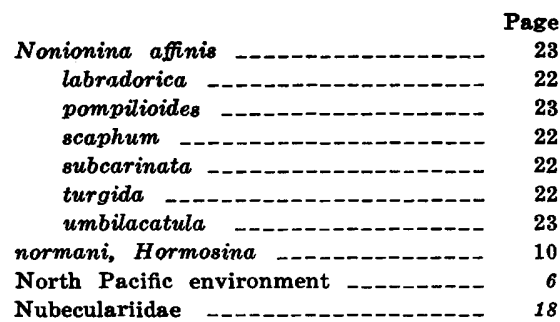

o

Oculosiphon linearis _._._-_._-_ 7,8 Ophthalmidium --_-_-_-_-_-_- 18 acutimargo _-_-_-_--- 6, 9, 11, 18 pusillum _-_._._._._. 6, 10, 18; pl. 3

orbiculare, Elphidium --_-_---_-_-_- T

Orbulina universa -

$\mathbf{P}$

pachyderma, Globigerina

pacifica, Globobulimina

papillata, Thurammina _-_-_-_-_ 1s, 16; pl. 1

pauciloculata, Virgulina --_-_-_ 8

peregrina, Euuvigerina --_---_-_ 20

Uvigerina _-_____- 6, 8, 11, 20; pl. 3 peruviana, Trochammina -..-_-_._ 17 pilulifer, Reophax (-..-- 6, 8, 14 pilulifera, Reophax - -

Pioneer, U.S. Coast and Geodetic Survey ship -_.-_ 1

Placopsilina - 16

bradyi -..-_-_-_ 9, 10, 16 confusa Plain, deep-sea --_-_-_-_-_-_-_- 7 plicata, Bolivina

Polystomella umbilicatula incerta

(Polystomella) magellanicum,

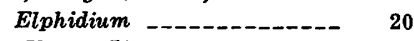

polystropha, Verneuilina pompilioides, Melonis _-_-_-- 6, 11, 23; pl. 4

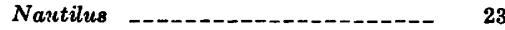

Nonion ---

Nonionina --_-

propinqua, Eggerella -_._-_-____._ Pl. 3

Proteonina difflugiformis -

Psammosiphonella discreta --_- 12

sp -_-_-_-_._-_ 7, 8, 9, 10, 11, 12

Psammosphaera -_._-_-_-_-__-_ 13

fusca -.-_-_-_- 10

rustica -

Pseudogaudryina atlantica _._._._._._ 6, 7 sp - - 7 pseudoplicata, Bolivina _._._-_._ 6, 7,19

Pseudopolymorphina lingua _-_.--_-_ 7

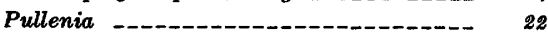
quinqueloba subcarinata _-__- 6, 8, 9, 10,11, 22; pl. 4

Pulvinulina elegans -.---------- 23 exigua -

Pulvinulinella umbonifera -....-... 20

pusillum, Ophthalmidium -_-_- 6, 10, 18; pl. 3

Spiroloculina -_-_-_-_ 18

Spirophthalmidium -.-.- 18

pygmaea, Uvigerina -_-_-_-_-_- 20

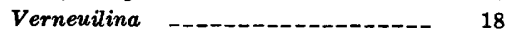

Pyrgo -

sp -

pyrula, Bulimina -

$\mathbf{Q}$

quinqueloba, Pullenia

22
Quinqueloculina

Page

$$
\text { sp }
$$

$\overline{6}, 10,19$

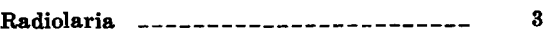
refulgens, Cibicides _-__-_-_-_-_ 6, 7

Reophax - - - dentalinaformis $\quad \ldots-6,8,9,11,12,18 ;$ pl. 1 diflugiformis $\quad--6,8,9,10,11,12,18$; pl. 1 distans - - excentricus _-_._-_-__-_ 6, 8,9,10

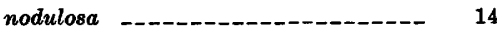
nodulosus _-_._- 6, 8, 9, 10,12, 14; pl. 1 pilulifer ---_-_-_-_-_--- 6, 8, 14 pilulifera -_-_- 14 scorpiurus ------_--- 6, 9, 10, 14; pl. 1 scotti sp - -

Rhabdammina abyssorum - 6, 12 discreta -_-_-_-_-_-_-_-_-_ 12 sp -

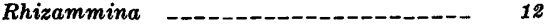
sp ____________- 6, 8, 12; pl. 1 ringens, Alveolophragmium --- 6, 8, 9, 10,15 Haplophragmoides -_-_-_-_-_--- 15 Trochammina --------------- 15 robusta, Bolivina -_-_-_-____-_ 6, 10,19 Rosalina sp --_-_-_-_-_-_-_ 6, 7 rosea, Discorbis _-_._-__-_-_-_-_ 9

Rotalia elegans -_-_-_-_-_-_-_-_-- 23 $\begin{array}{cc}\text { Rotalina lamarckiana - } & 22 \\ \text { turgida - } & \end{array}$ rustica, Psammosphaera _._-_._-_.- 6,13

\section{$\mathbf{s}$} sphaerica sp

Saccamminidae --_-

Sample collection -

Sampling procedure

scabra, Bulimina --Eggerella --

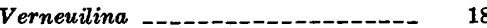
scapha, Nonion -_-_-_-_-_-_ 22 scaphum, Nonion --_-_-_-_-- 6, 7, 22 Nonionina -.-_-_-_-_-_-_-_ 22 scitulum, Alvelophragmium_ 6, 8, 9, 12, 15; pl. 1 Haplophragmium -_-_-_-_-_- 15 scorpiurus, Reophax ---- 6, 9, 10,14; pl. 1

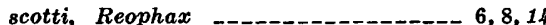
seminuda, Buliminella elegantissima --- 19 sphaerica, Saccammina ---_----- 6, 9,10,19 Spirolina agglutinans ----_---_-_--- 16

Spiroloculina acutimargo _...-.--_-. 18

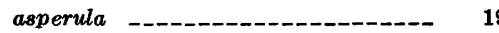
pusillum --_-_-_- 18

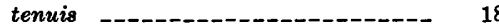
sp -

Spirophthalmidium acutimargo --_--- 18 pusillum -..

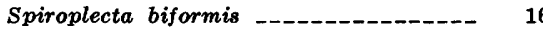
Spiroplectammina biformis -_-_-_-_- 6, 8, 9, 10, 16; pl. 2 squamata, gordialis Trochammina -..-. 13 subcarinata, Nonionina --_-_-_-_- 22 Pullenia --_._-. 6, 8, 9, 10, 11, 22; pl. 4 subglobosa, Cassidulina --_-- 6, 8, 11, 21; pl. 4 Labrospira --_-_-_-_-_-_-_-_ 15 subglobosum Alveolophragmium --_- 6 , $7,8,9,10,11,12,15 ; \mathrm{pl}$. subrotunda, Miliolinella_ 6, 8,9,10,11, 19; pl. 3

\section{$\mathbf{T}$}

Page

tenuimargo, Ammoscalaria --_--_-_--- 10,11 tenuis, Ammodiscus -_._._-_-_._- 22 Involutina _-...- 6, 8, 9, 10, 22; pl. 4 Spiroloculina --_-_-_-_- 18 teretis, Cassidulina -..._-_-_-_-_ 6, 7, 21 Textularia agglutinans biformis _.-.-- 16 Textulariidae -.-_- 16 Thurammina -_-_-_-_-_-_-_-_ 8, 9, $1 \mathrm{~s}$ papillata tortuosa, Cassidulina -_-_._-_-_ 6, 7, 21 triangularis, conica Valvulina --_---- 18 Tritaxis conica
Trochammina
charlothio charlottenis --_-_-_-_-_-_--_ 10 galeata - globigeriniformis -- 7, 8, 9, 10, 11. 17; pl. 3 grisea _.______ $7,8,9,10,17 ;$ pl. 2 inflata kellettae -_malovensis nana -_-__-_._-_ 7, 9, 17; pl. 2 nitida -_-_-_-_-- 7,9, 10, 11, 12, 17; pl. 2 peruviana ringens ---_-_-_-_-_-_--_- 15 squamata gordialis --.---_--_-- 13 trullissata _._._. sp - -

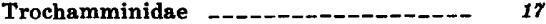
trullissata, Cyclammina $6,8,9,10,11,14 ;$ pl. 1 Trochammina --_-_-_-_ 14, 15

Truncatulina bradyi -.-.--_-_--_-_- 21

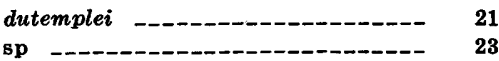
sp -

Haplophragmium -.-.-- 17 turgida, Nonionella ---_---- 6, 8, 22; pl. 4 Nonionina -

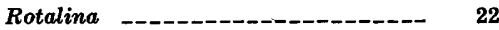
Turrilinidae - -

\section{U}

umbilacatula, Nonionina --_----_-_-- 23 umbilicatula incerta, Polystomella -.- 20 umbonifera, Epistominella _-_-_ 6, 9, 20; pl. 3 Pulvinulinella -_-_-_-_-_-_- 20 universa, Orbulina -...- 8 Uvigerina - angulosa -..- 20 cushmani -_._-_._-_._-_.-- 6, 7, 20 peregrina pygmaea -.---_-

Uvigerinidae - -

\section{$\mathbf{v}$}

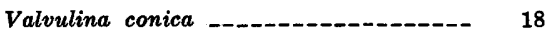
triangularis conica - 18

Verneuilina bradyi --_-_-_-_-_---- 18 polystropha pygmaea - scabra -

Virgulina complanata --_-_-_-_- 6, 7

$\begin{array}{rr}\text { pauciloculata } & 8 \\ \text { voluta, Globigerinita } & -\end{array}$

w

weisneri, Alveolophragmium 6, Labrospira _-._-_._._. 15.

U.S. GOVERNMENT PRINTING OFFICE : 1973 0-495-617 


\section{PLATES $1-4$}

Contact photographs of the plates in this report are available, at cost, from

U.S. Geological Survey Library, Federal Center, Denver, Colorado 80225. 


\section{PLATE 1}

Figure 1. Nodellum membranaceum (Brady). $\times 115$, sample P-19-61, USNM 175142.

2. Rhizammina? sp. (p. 12) $\times 68$, sample P-12-61, USNM 175143.

3. Marsipella cylindrica Brady (p. 12). $\times 70$, sample P-13-61, USNM 175144

4. Bathysiphon discreta (Brady) (p. 12). $\times 73$, sample P-19-61, USNM 175145.

5. Jaculella acuta Brady (p. 12). $\times 190$, sample P-41-61, USNM 175146.

6. Hyperammina friabilis Brady (p. 12). $\times 23$, sample P-41-61, USNM 175147 .

7. Thurammina papillata Brady (p. 13). $\times 80$, sample P-48-61, USNM 175148.

8. Glomospira gordialis (Jones and Parker) (p. 13). a, Side view; $b$, end view; $\times 43$, sample P-40-61, USNM 175149.

9. Reophax dentalinaformis Brady (p. 13). $\times$ 55, sample P-56-61, USNM 175150.

10. Reophax difflugiformis Brady (p. 13). $\times 436$, sample P-41-61, USNM 175151.

11. Reophax distans Brady (p. 13). $\times 62$, sample P-33-61, USNM 175152.

12. Reophax nodulosus Brady (p. 14). $\times 18$, sample P-40-61, USNM 175153.

13. Reophax scorpiurus de Montfort (p. 14). $\times 37$, sample P-41-61, USNM 175154 .

14. Adercotryma glomeratum (Brady) (p. 14). $\times 214$, sample P-41-61, USNM 175155 .

15. Cyclammina trullissata (Brady) (p. 14).

a, Side view; b. apertural view; $\times 73$, sample P-40-61, USNM 175156.

16. Alveolophragmium nitidum (Göes) (p. 15).

a, Side view; b, apertural view; $\times 200$, sample P-19-61, USNM 175157.

17. Alveolophragmium cf. A. nitidum (Göes) (p. 15).

a, Side view; b. apertural view; $\times$ 185, sample P-41-61, USNM 175158.

18. Cyclammina cancellata Brady (p. 14). $\times 19$, sample P-40-61, USNM 175159.

19. Alveolophragmium scitulum (Brady) (p. 15).

a, Apertural view; b. side view; $\times 102$, sample P-19-61, USNM 175160.

20. Alveolophragmium subglobosum (G. O. Sars) (p. 15).

a, Side view; $b$, peripheral view; $\times$ 87, sample P-19-61, USNM 175161. 


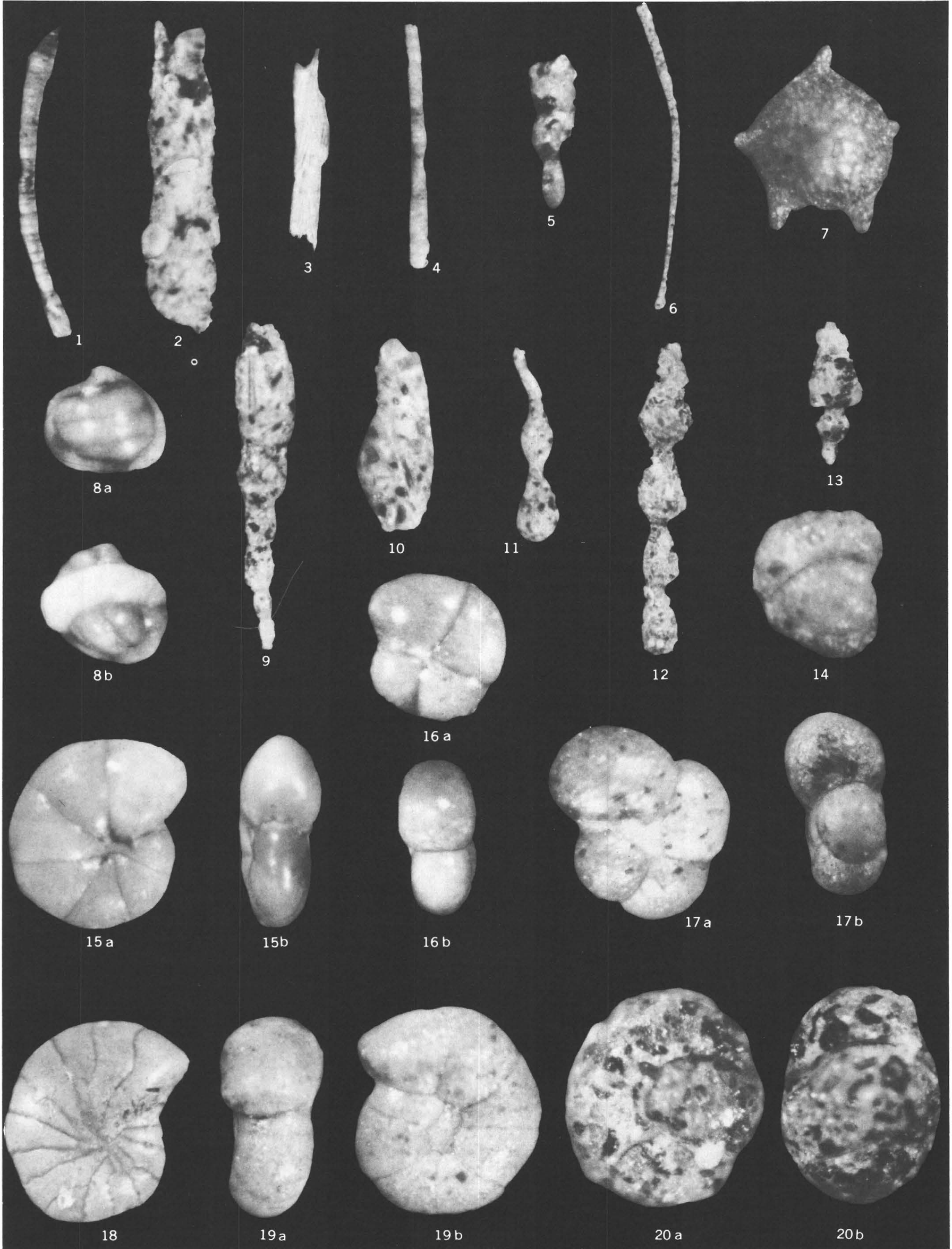




\section{PLATE 2}

FigURE 1. Alveolophragmium weisneri (Parr) (p. 15).

a, Side view; b, apertural view; × 155, sample P-19--61, USNM 175162.

2. Alveolophragmium? sp. (p. 15).

$\times 195$, sample P-49-61, USNM 175163.

3. Ammobaculites agglutinans (d'Orbigny) (p. 16). $\times 61$, sample P-41-61, USNM 175164.

4. Ammobaculites agglutinans filaformis Heron-Allen and Earland (smooth form) (p. 16).

$\times 64$ sample P-41-61, USNM 175165.

5. Ammobaculites agglutinans filaformis Heron-Allen and Earland (rough form) (p. 16)

$\times 106$, sample P-49-61, USNM 175166.

6. Ammomarginulina foliacea (Brady) (p. 16) $\times 137$, sample P-38-61, USNM 175167.

7. Spiroplectammina biformis (Parker and Jones) (p. 16). $\times 180$, sample P-49-61, USNM 175168 .

8. Bigenerina minutissima Earland (p. 16). $\times 172$, sample P-49-61, USNM 175169.

9. Trochammina grisea Heron-Allen and Earland (p. 17).

a, Evolute side view; b, apertural view; $c$, involute side view; $\times 154$, sample P-19-61, USNM 175170.

10. Trochammina inflata (Montagu) (p. 17).

a, Evolute side view; b, involute side view; $\times 182$, sample P-48-61, USNM 175171.

11. Trochammina ef. T. malovensis Heron-Allen and Earland (p. 17).

a, Evolute side; b, involute side; $\times 252$, sample P-41-61, USNM 175172.

12. Trochammina kellettae Thalmann (p. 17).

a, Evolute side; b, involute side; $\times 240$, sample P-41-61, USNM 175173.

13. Trochammina malovensis Heron-Allen and Earland (p. 17).

a, Involute side; b, evolute side; $\times 320$, sample P-12-61, USNM 175174.

14. Trochammina nitida Brady (p. 17).

a, Evolute side; b, involute side; $\times 220$, sample P-40-61, USNM 175175.

15. Trochammina nana (Brady) (p. 17).

a, Involute side; b, evolute side; $\times 215$, sample P-49-61, USNM 175176. 

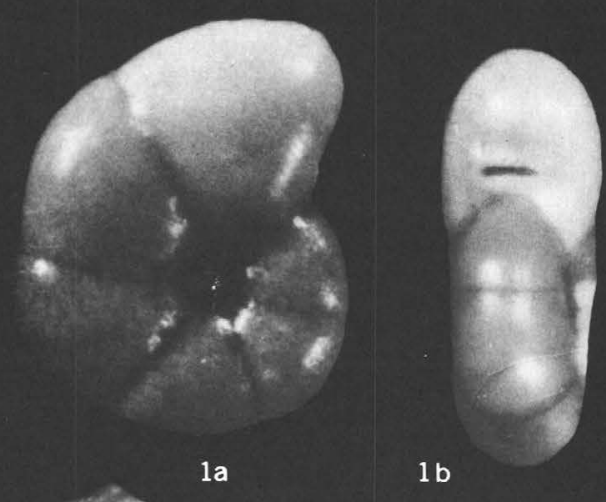
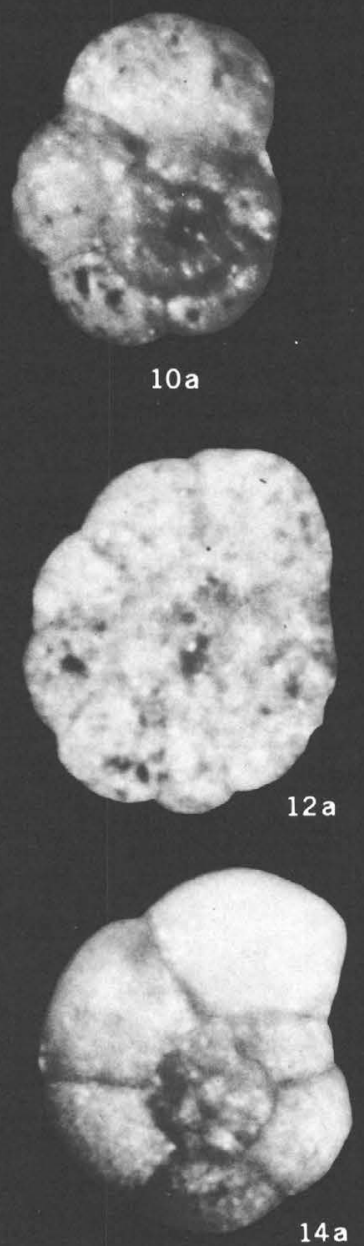

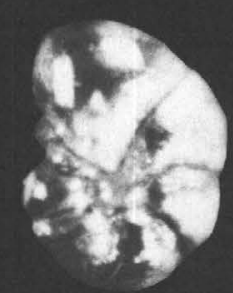

2
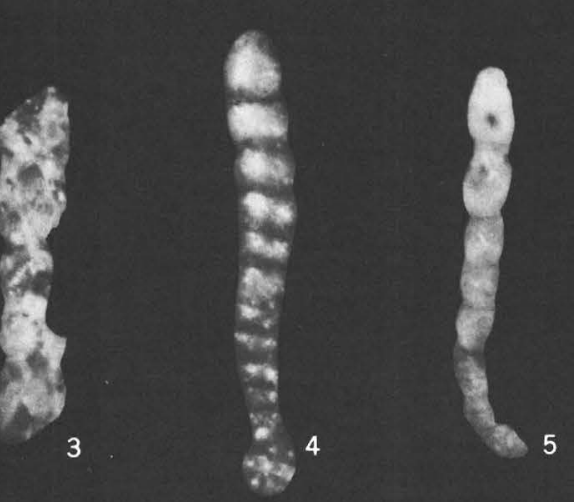

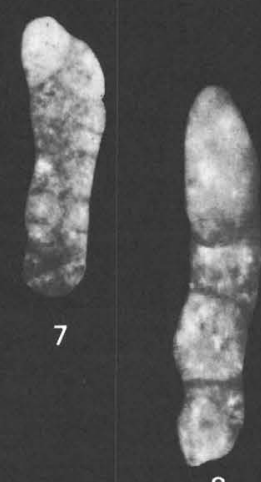

6

$10 \mathrm{a}$

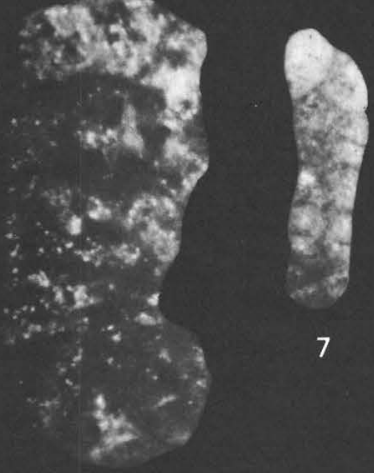

8

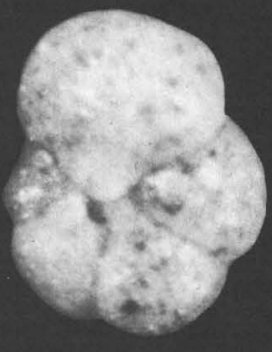

$10 \mathrm{~b}$
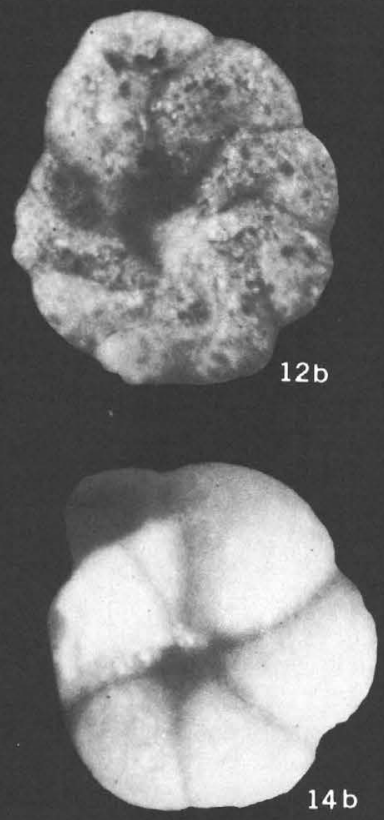

3

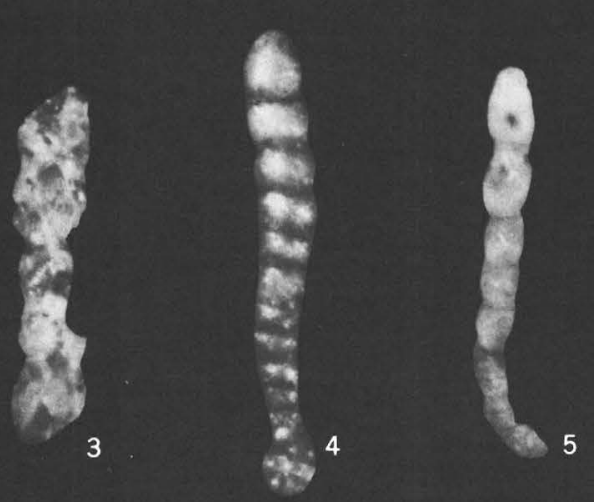




\section{PLATE 3}

FIGURE 1. Trochammina globigeriniformis (Parker and Jones) (p. 17).

a, Evolute side; b, involute (apertural) side; $\times$ 174, sample P-41-61, USNM 175177.

2. Cystammina galeata (Brady) (p. 18).

a, b, Side views; $\times$ 83, sample P-19-61, USNM 175178.

3. Dorothia exilis Cushman (p. 18).

$\times 190$, sample P-49-61, USNM 175179.

4. Eggerella bradyi (Cushman) (p. 18).

a, Apertural view; b, side view; × 138, sample P-19-61, USNM 175180.

5. Eggerella scabra (Williamson) (p. 18).

a, Apertural view; b, side view; $\times$ 380, sample P-48-61, USNM 175181.

6. Eggerella propinqua (Brady).

a, Apertural view; b, side view; × 204, sample P-6-61, USNM 175182.

7. Ophthalmidium pusillum (Earland) (p. 18).

$\times 113$, sample P-32-61, USNM 175183.

8. Miliolinella subrotunda (Montagu) (p. 19).

$\times 166$, sample P-49-61, USNM 175184.

9. Bulimina aculeata d'Orbigny (p. 19).

$\times$ 56, sample P-16-61, USNM 175185.

10. Globobulimina ef. Bulimina auriculata Bailey (p. 20).

$\times$ 47, sample P-16-61, USNM 175186.

11. Uvigerina peregrina Cushman (p. 20)

$\times 106$, sample P-16-61, USNM 175187.

12. Epistominella exigua (Brady) (p. 20).

a, Evolute side; b, involute side; $\times 360$, sample P-49-61, USNM 175188.

13. Epistominella umbonifera (Cushman) (p. 20).

a, Evolute side; b, apertural view; c, involute side; $\times 97$, sample P-49-61, USNM 175189. 


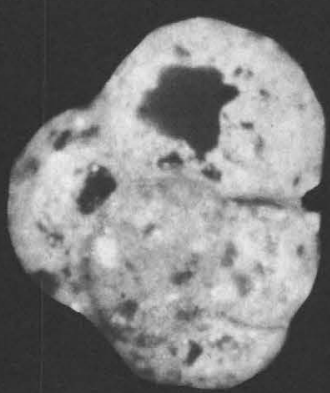

1 a

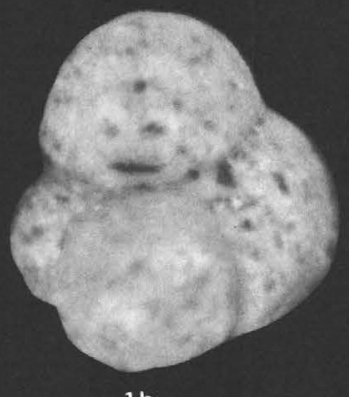

$1 b$
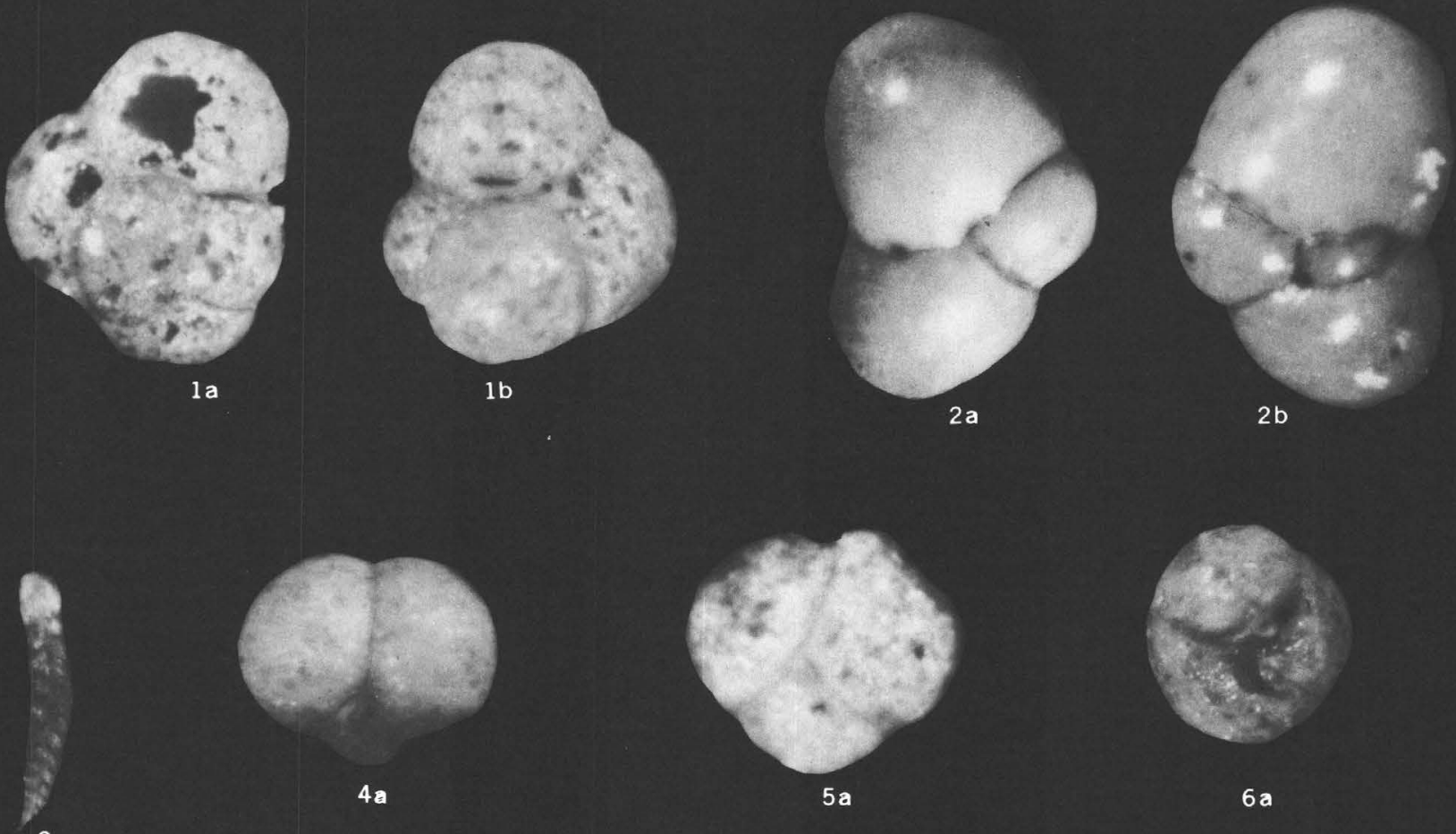

$4 a$
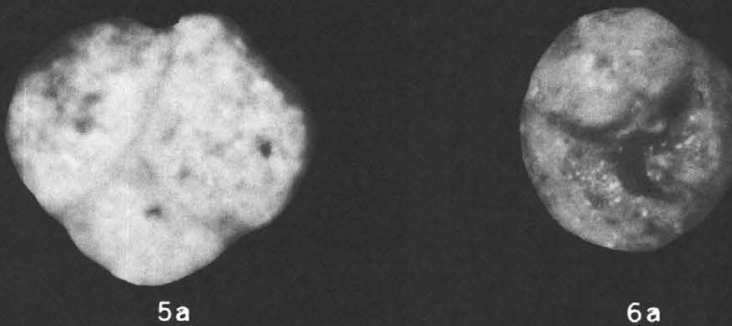

3

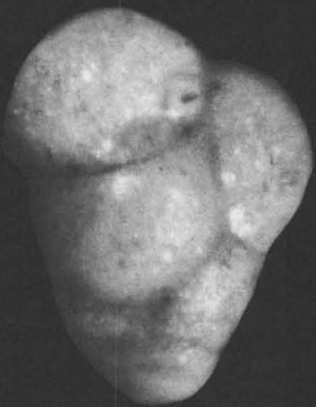

$4 \mathrm{~b}$

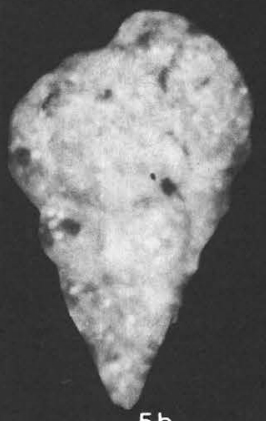

$5 b$
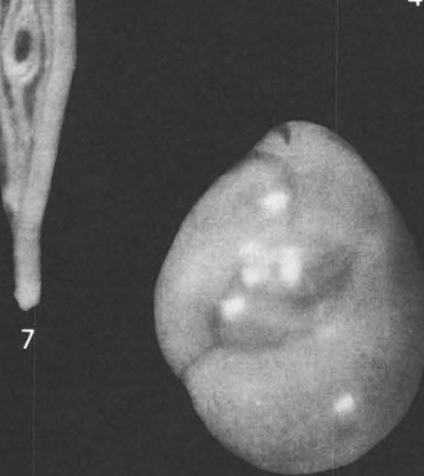

8
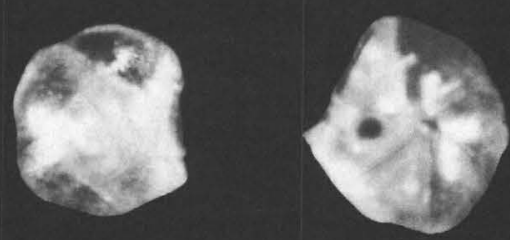

$12 \mathrm{a}$

$12 \mathrm{~b}$

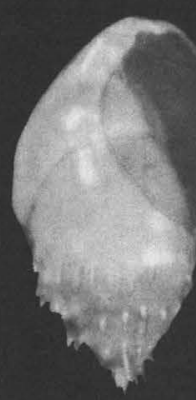

9

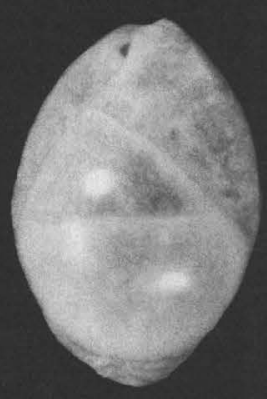

10

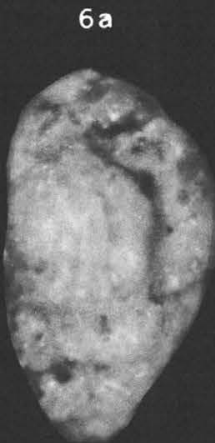

$6 b$

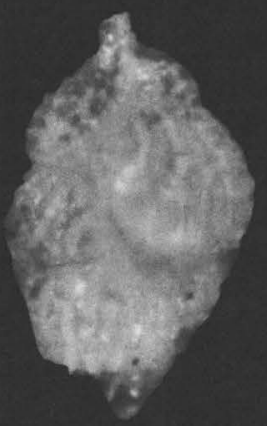

11

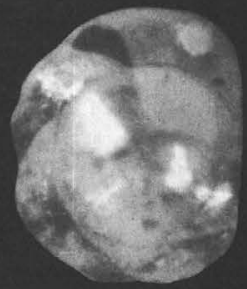

$13 a$
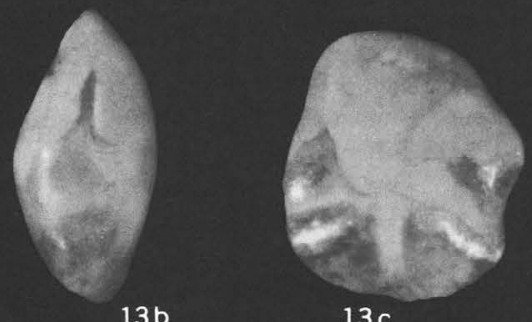


\section{PLATE 4}

Figure 1. Elphidiella groenlandica (Cushman) (p. 20).

a, Apertural view; b, side view; $\times$ 79, sample P-16-61, USNM 175190.

2. Cibicides bradyi (Trauth) (p. 21).

a, Involute side; b, evolute side; × 227, sample P-19-61; USNM 175191.

3. Cassidulina subglobosa Brady (p. 21).

$\times 210$, sample P-29-61, USNM 175192.

4. Nonion labradoricum Dawson (p. 22).

a, Apertural view; b, side view; × 100, sample P-16-61, USNM 175193.

5. Ehrenbergina hystrix Brady (p. 21).

$\times 78$, sample P-49-61, USNM 175194.

6. Involutina tenuis (Brady) (p. 22).

$\times 134$, sample P-41-61, USNM 175195.

7. Gyroidina lamarckiana (d'Orbigny) (p. 22)

a, Evolute side; b, peripheral (apertural) view; c, involute side; $\times 122$, sample P-49-61, USNM 175196.

8. Nonionella turgida (Williamson) (p. 22).

a, Evolute side; b, involute side; $\times$ 190, sample P-16-61, USNM 175197.

9. Pullenia subcarinata (d'Orbigny) (p. 22)

a, Peripheral view; b, side view; × 142, sample P-12-61, USNM 175198.

10. Anomalina globulosa Chapman and Parr (p. 22)

a, Evolute side; b, peripheral view; c, involute side; $\times 109$, sample P-12-61, USNM 175199.

11. Melonis pompilioides (Fichtel and Moll) (p. 23).

a, Side view; b, peripheral view; × 160, sample P-29-61, USNM 179200.

12. Melonis affine (Reuss) (p. 23).

$\times 200$, sample P-49-61, USNM 179201.

13. Cibicidoides cf. C. mundulus (Brady, Parker, and Jones) (p. 23).

a, Evolute side; b, involute side; $\times$ 280, sample P-49-61, USNM 179202. 


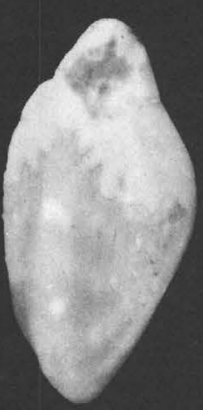

$1 \mathrm{a}$

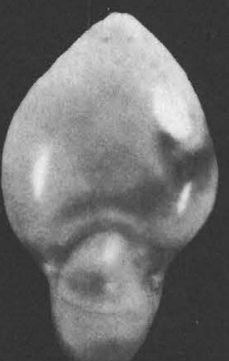

$4 a$

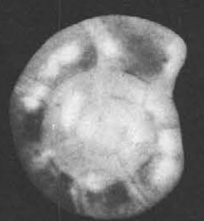

$7 a$

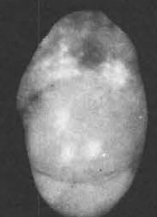

$9 a$

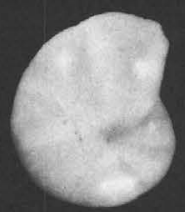

$11 \mathrm{a}$

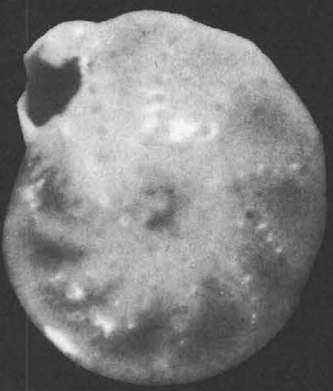

$1 b$

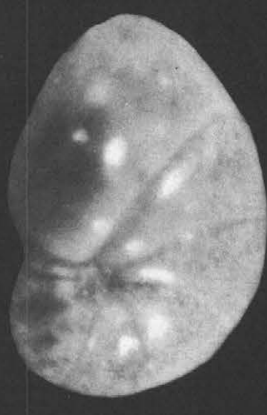

$4 b$

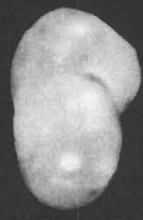

$7 b$

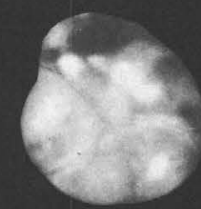

$9 \mathrm{~b}$

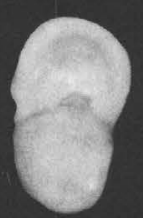

$11 \mathrm{~b}$

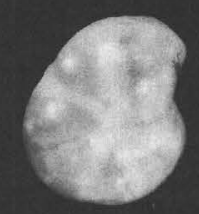

$2 a$
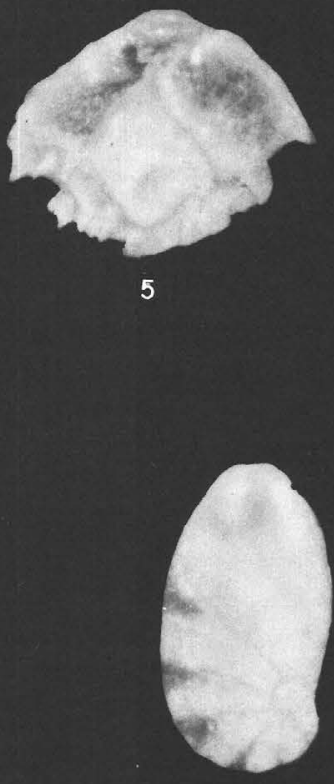

$8 \mathbf{a}$

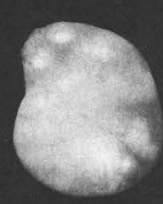

$2 b$

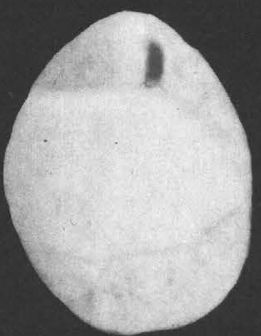

3
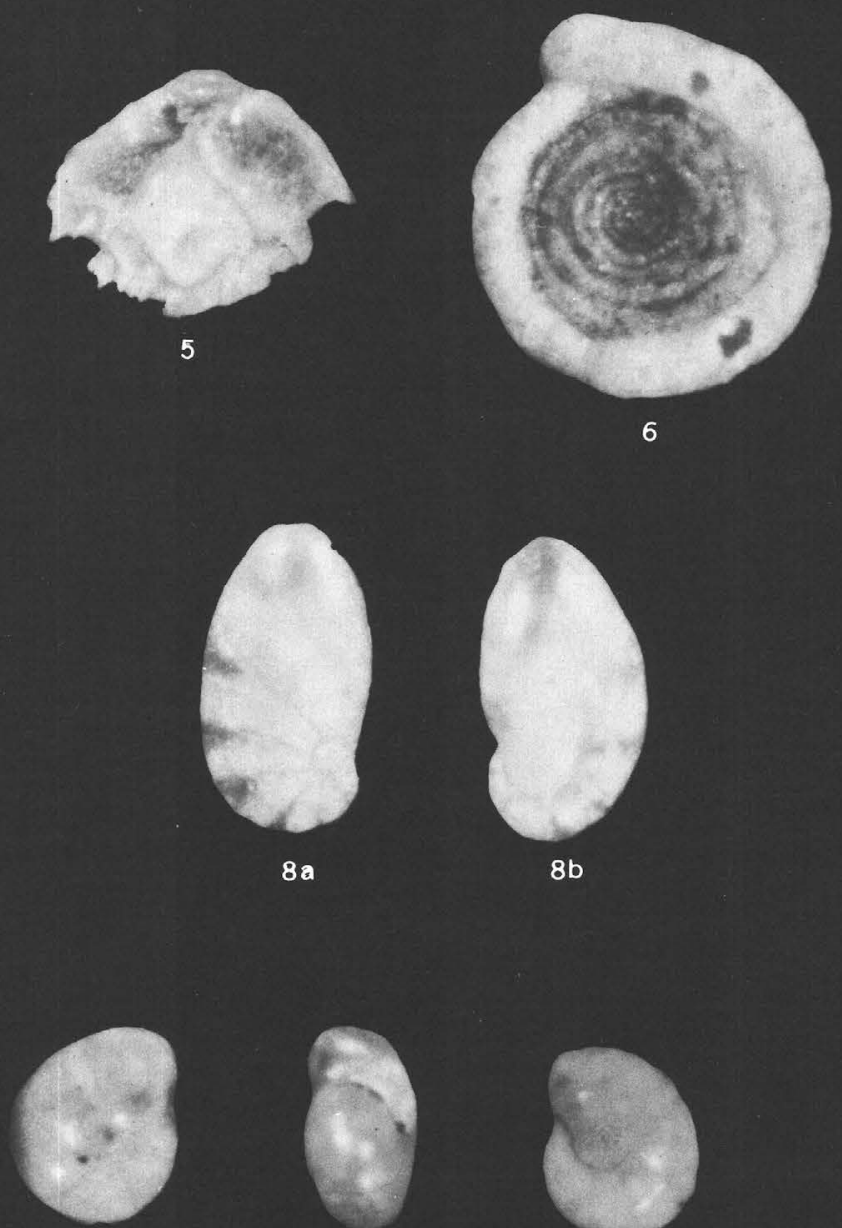

$10 \mathrm{a}$

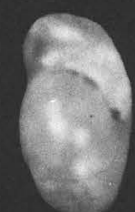

$10 \mathrm{~b}$
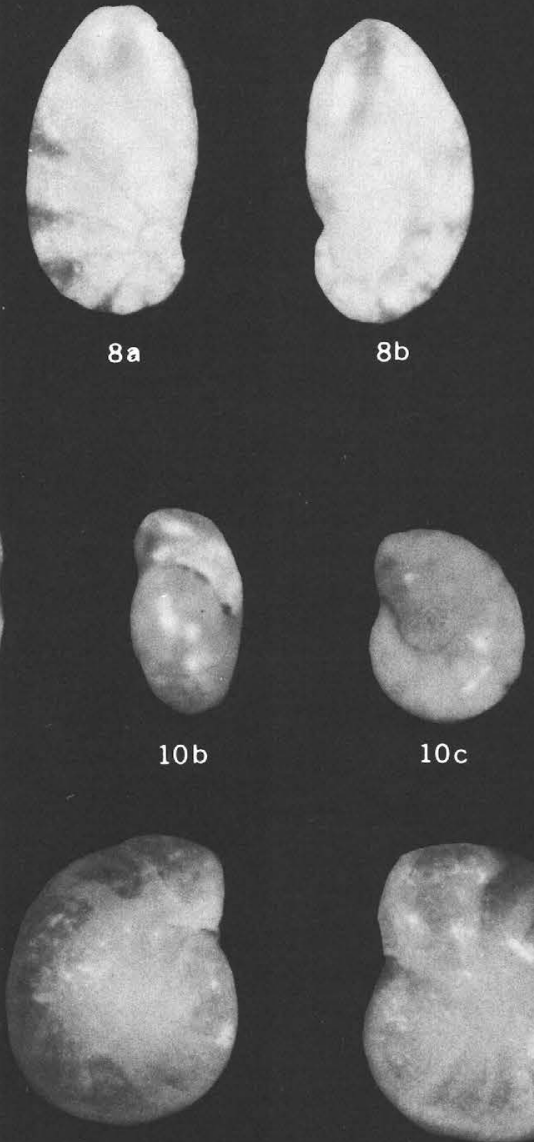

$13 a$

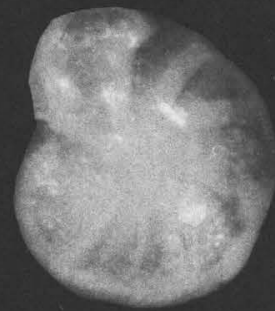

$13 \mathrm{~b}$ 


